\title{
Post-flood monitoring in a sub-tropical estuary and benchmarking with PFASs allows measurement of chemical persistence on the scale of months
}

Rodrigo Álvarez-Ruiz ${ }^{*}, 1,2$ Darryl W. Hawker ${ }^{2,3}$, Jochen F. Mueller², Michael Gallen ${ }^{2}$, Sarit Kaserzon ${ }^{2}$, Yolanda Picó ${ }^{1}$ and Michael S. McLachlan ${ }^{4}$

${ }^{1}$ Food and Environmental Safety Research Group (SAMA-UV), Desertification Research Centre (CIDE-UV, GV, CSIC), Moncada-Naquera Road km 4.5, 46113 Moncada, Valencia, Spain.

${ }^{2}$ The University of Queensland, Queensland Alliance for Environmental Health Sciences (QAEHS), 20 Cornwall Street, Woolloongabba, QLD 4102, Australia

${ }^{3}$ Griffith School of Environment and Science, Griffith University, Nathan, QLD 4111, Australia

${ }^{4}$ Department of Environmental Science (ACES), Stockholm University, Sweden

Table of contents

\begin{tabular}{|l|l|}
\hline Text S-1. Reagents & $\mathrm{S}-4$ \\
\hline Text S-2. Extraction and analysis & $\mathrm{S}-5$ \\
\hline Text S-3. Quality assurance & $\mathrm{S}-7$ \\
\hline Text S-4. Fitting models with variable reaction rate constants & $\mathrm{S}-8$ \\
\hline Text S-5. Hydrological and meteorological considerations & $\mathrm{S}-9$ \\
\hline Text S-6. Interpretation of temporal concentration profiles & $\mathrm{S}-10$ \\
\hline & \\
\hline Table S-1. Sample collection dates and times. & $\mathrm{S}-17$ \\
\hline Table S-2. MS and quality assurance information for PFASs. & $\mathrm{S}-18$ \\
\hline Table S-3. MS information for labelled PFAS standards. & $\mathrm{S}-20$ \\
\hline
\end{tabular}

* Corresponding author

Phone: +34-963-543-092 / +34-963-424-216

E-mail: Rodrigo.Alvarez@uv.es 
Table S-4. MS information for PFAS Recovery standards MS information.

Table S-5. MS and quality assurance information for PPCPs and pesticides analysed in ESI positive mode.

Table S-6. MS and quality assurance information for PPCPs and pesticides analysed in ESI negative mode.

Table S-7. MS information for labelled PPCP and pesticide standards.

Table S-8. Daily PFASs discharge from Goodna WWTP in the upper stretch of the Brisbane River estuary.

Table S-9. Daily PPCPs and pesticides discharge from Goodna WWTP in the upper stretch of the Brisbane River estuary.

Table S-10. PFAS concentrations in surface water samples.

Table S-11. PPCP and pesticide concentrations in surface water samples.

Table S-12. Rate constant for chemical loss from the estuary segment and emission flux estimated
by fitting the measured concentrations in river water to Eq. 2 (time invariant rate constant) for

Type B compounds.

Table S-13. Rate constant for chemical loss from the estuary segment and emission flux estimated by fitting the measured concentrations in river water to Eq. 5 (UV-radiation intensity dependant rate constant) for Type B compounds. Presented half-lives are based on the mean of the daily UV indices when water samples were taken.

Table S-14. Rate constant for chemical loss from the estuary segment and emission flux estimated by fitting the measured concentrations in river water to Eq. 7 (temperature dependant) for Type B compounds. Presented half-lives are based on the mean of the daily water temperatures when water samples were taken.

Table S-15. Rate constant for chemical loss from the estuary segment derived by fitting the measured concentrations in river water to Eq. 4 (C: time invariant rate constant), Eq. 6 (UV: UVradiation dependant constant). Eq. 8 ( $\mathrm{T}^{\mathrm{a}}$ : Temperature dependant constant) for Type $\mathrm{C}$ compounds. Presented half-lives for the UV-and temperature models are based on the mean of the daily UV indices or water temperatures when water samples were taken.

Figure S-1. Upper Panel: Cumulative rainfall at Savage's Crossing gauging station between March and December 2017 [1]. Lower Panel: Example of tidal amplitude of the Brisbane River at Moggill between April 5 and April 12, 2020.

Figure S-2. Concentration of Type A chemicals during the whole sampling period. The X-axis labels mark the beginning of the month. A zoom to the $y$-axis is provided for some substances to facilitate inspection of the trend.

Figure S-3. Concentration of Type B chemicals during the whole sampling period. The X-axis labels mark the beginning of the month. 
Figure S-4. Concentration of Type C chemicals during the whole sampling period. A semilogarithmic plot is provided for some substances to facilitate inspection of the elimination kinetics. The $\mathrm{x}$-axis labels mark the beginning of the month.

Figure S-5. Concentration trend during the whole sampling period for chemicals showing no consistent time trend. The $\mathrm{x}$-axis labels mark the beginning of the month.

Figure S-6. Upper panel: Daily UV index at the Brisbane UV monitoring station, summarized from hourly UV index data [2] Lower panel: Daily average water temperature at the Savage's Crossing station on the Brisbane River [1]. The time axis of both graphs extends from April 7 (day 0) to October 22 (day 200) 2017. 


\section{Text S-1. Reagents}

All reagents and analytical standards were of high purity. $\mathrm{MeOH}$ ( $\mathrm{LiChrosolv}{ }^{\circledR} \mathrm{LC}$ grade $\geq 99.8 \%$ ) was purchased from MERCK (Bayswater, VIC, Australia). Ammonium hydroxide solution 28.0-30.0\% was from Sigma Aldrich (Bayswater, VIC, Australia).

The PFASs analysed for were: perfluorobutanoic acid (PFBA), perfluoropentanoic acid (PFPeA), perfluorohexanoic acid (PFHxA), perfluoroheptanoic acid (PFHpA), perfluorooctanoic acid (PFOA), perfluorononanoic acid (PFNA), perfluorodecanoic acid (PFDA), perfluoroundecanoic acid (PFUnDA), perfluorododecanoic acid (PFDoDA), perfluorotridecanoic acid (PFTrDA), perfluorotetradecanoic acid (PFTeDA), perfluorohexadecanoic acid (PFHxDA), perfluorooctadecanoic acid (PFODA), perfluorobutanesulphonate (PFBS), perfluoropentanesulsulphonate (PFPeS), perfluorohexanesulsulphonate (PFHxS), perfluoroheptanesulphonate (PFHpS), perfluorooctanesulsulphonate (PFOS), perfluorononanesulfonate (PFNS), perfluorodecanesulfonic acid (PFDS), perfluordodecanesulphonate (PFDoDS), perfluorooctanesulfonamide (FOSA), perfluorooctanesulfonamide acetic acid (FOSAA), N-ethyl perfluorooctanesulfonamide (N-Et-

FOSA), N-ethyl perfluorooctanesulfonamidoacetic acid (N-EtFOSAA), Nethyl perfluorooctanesulfonamidoethanol (N-EtFOSE), N-methyl fluorooctanesulfonamide (N-MeFOSA), N-methyl perfluorooctanesulfonamidoacetic acid (N-MeFOSAA), N-methyl perfluorooctanesulfonamidoethanol (N-MeFOSE), perfluoroethylcyclohexanesulfonate (PFECHS), the fluorotelomer sulfonates (FTS): 10:2 FTS, 8:2 FTS, 6:2 FTS, and 4:2 FTS and the perfluoroalkyl phosphate esters (PAP): 8:2 PAP, 6:2 PAP, SAmPAP, 8:2 DiPAP, 6:2 DiPAP and 6:2 8:2 DiPAP. A mixture of ${ }^{13} \mathrm{C}$ and ${ }^{18} \mathrm{O}$ labelled PFASs (purchased from Wellington Laboratories, Guelph, Ontario, Canada) served as surrogate internal standards (ISs) to correct for method recovery (Table S-3). ${ }^{13} \mathrm{C}_{8}$-PFOA and ${ }^{13} \mathrm{C}_{8}$-PFOS were used as instrument performance standards to check for correct functioning of the instruments (Table S-4).

The PPCPs and pesticides analysed for were: salicylic acid, acesulfame K, (4-Chloro-2-methylphenoxy) acetic acid (MCPA), ibuprofen, 2,4-Dichlorophenoxyacetic acid (2,4-D), dicamba, 4-(2,4-dichlorophenoxy)butyric acid (2,4-DB), 2,4,5-Trichlorophenoxyacetic acid (2,4,5-T), triclopyr, bromoxynil, triclosan hydrochlorothiazide, furosemide, diketonitrile, paracetamol, 3,4 dichloroaniline, methomyl, nicotine, gabapentin, desisopropyl atrazine, cotinine, paraxanthine, simazine hydroxyl, desethyl atrazine, clopyralid, 5-hydroxyindoleacetic acid (5HIAA), N,N-Diethyl-meta-toluamide (DEET), hydroxycotinine, caffeine, ametryn hydroxyl, pyrimethanil, terbuthylazine desethyl, simazine, desdimethyl diuron (DCPU), propoxur, metribuzin, atrazine, desmethyl diuron (DCPMU), dichlorvos, carbofuran, methiocarb, ametryn, tebuthiuron, propazine, terbuthylazine, asulam, naproxen, diuron, fluometuron, carbamazepine, prometryn, terbutryn, picloram, hexazinone, fluroxypyr, imidacloprid, bromacil, tramadol, atenolol, desmethyldiazepam, 
imazapic, venlafaxine, metalaxyl, pendimethalin, metolachlor, imazethapyr, codeine, temazepam, fenamiphos, diazinon, tebuconazole, fluoxetine, desmethyl citalopram, citalopram, fluazifop, malathion, propiconazole, prothioconazole, chlorpyriphos, haloxyfop, metsulfuron-methyl, tadalafil, verapamil, sildenafil, atorvastatin, iopromide and haloxyfop-methyl. A total of 48 ISs were used for their quantification (Table S7): 2,4-D-13C6, 5HIAA-D2, acesulfame-D4, atenolol-D7, atorvastatin-D5, atrazine-D5, atrazine desisopropyl-D5, bromacil-D3, caffeine-13C3, carbamazepine-D10, carbofuran-D3, citalopram-D6, codeine-D3, cotinine-D3, DEET-D7, desethyl atrazine-D6, diazinon-D10, diuron-D6, fluazifop-D4, fluoxetine-D6, gabapentin-D10, haloxyfop-D4, hexazinone-D6, hydrochlorothiazide-13CD2, hydroxycotinine-D3, ibuprofen-D3, imidacloprid-D4, iopromide-D3, MCPA-D6, methiocarb-D3, methomyl-D3, metolachlor-D6, metribuzin-D3, metsulfuron-methyl-D3, nicotine-D4, paracetamol-D4, paraxanthine-D3, prometryn-D6, propazine-D6, propiconazole-D5, propoxur-D3, simazine-D10, tebuconazole-D6, tebuthylazine desethylD9, temazepam-D5, terbuthylazine-D5, tramadol-D6 and venlafaxine-D6.

For the PPCPs, pesticides and PFASs for which IS analogues were not available, quantification was done using external calibration or the IS with the most similar structure (Tables S-2, S-5 and S-6).

\section{Text S-2. Extraction and analysis}

\section{Extraction procedure for PPCPs and pesticides}

Samples were extracted using solid phase extraction (SPE) in a RESTEK manifold. Prior to extraction, $150 \mathrm{~mL}$ of surface water sample (50 mL for wastewater) was spiked with $10 \mu \mathrm{L}$ of a $1 \mu \mathrm{g} / \mathrm{mL}$ IS mix and the $\mathrm{pH}$ recorded ( $\mathrm{pH}$ was 7 to 8 for surface water samples). The SPE cartridges used were Strata ${ }^{\mathrm{TM}}-\mathrm{X} 33 \mu \mathrm{m}$ Polymeric reversed phase, $200 \mathrm{mg} / 6 \mathrm{~mL}$, from Phenomenex ${ }^{\circledR}$, conditioned with $4 \mathrm{~mL}$ of $\mathrm{MeOH}$ followed by $4 \mathrm{~mL}$ of MilliQ water. Samples were first shaken to ensure homogenization, and then passed through the cartridges dropwise. The cartridges were washed with $4 \mathrm{~mL}$ of MilliQ water, dried under vacuum for $30 \mathrm{~min}$ and then eluted using $2 \mathrm{~mL}$ of methanol dripped through the cartridge by gravity flow. This elution procedure was repeated, obtaining in total $4 \mathrm{~mL}$ of extract, which was collected in glass Falcon tubes and blown down to approximately $1 \mathrm{~mL}$ using a $40{ }^{\circ} \mathrm{C}$ heating plate and a gentle stream of nitrogen in a RATEK blowing down unit. The extracts were then filtered using PTFE $(0.2 \mu \mathrm{m})$ Captiva filters (from Agilent technologies, USA), preconditioned with $1 \mathrm{~mL}$ of $\mathrm{MeOH}$ and blown down again to a final volume of $50 \mu \mathrm{L}$. Finally, 200 $\mu \mathrm{L}$ of MilliQ water was added to obtain a final solvent mixture of 20: $80 \mathrm{MeOH}: \mathrm{H}_{2} \mathrm{O}$. The final extracts were stored on amber vials and refrigerated until analysis. 


\section{Extraction procedure for PFASs}

This extraction procedure was based on previous literature [3, 4], with some modifications. Briefly, the samples were also extracted using SPE. The sample volume was the same as for PPCPs and pesticides, and they were also spiked with $10 \mu \mathrm{L}$ of a $0.2 \mu \mathrm{g} / \mathrm{mL}$ IS mix. The SPE cartridges (Phenomenex ${ }^{\circledR} \operatorname{Strata}^{\mathrm{TM}} \mathrm{X}-$ AW $33 \mu \mathrm{m}$ Polymeric weak anion exchange, $100 \mathrm{mg} / 6 \mathrm{~mL}$ ) were conditioned using $4 \mathrm{~mL}$ of $0.2 \%$ ammonium hydroxide: $\mathrm{MeOH}$, followed by $4 \mathrm{~mL}$ of $\mathrm{MeOH}$ and $4 \mathrm{~mL}$ of MilliQ water. The samples were passed through the cartridge and then washed as for PPCPs and pesticides. Then, they were dried with vacuum for at least 3 hours. The cartridge was eluted with $4 \mathrm{~mL}$ of $0.2 \%$ ammonia: $\mathrm{MeOH}$, dripped through the cartridge by gravity flow. The extract was placed in Falcon tubes and blown down to a final volume of $80 \mu \mathrm{L}$, then $120 \mu \mathrm{L}$ of aqueous mobile phase (A) was added, affording a final solvent mixture of 40: $60 \mathrm{MeOH}: \mathrm{H}_{2} \mathrm{O}$. Then, $10 \mu \mathrm{L}$ of $0.2 \mu \mathrm{g} / \mathrm{mL}$ Recovery Standards (Table S-4) mixture was added. Finally, the extract was stored in a plastic vial and refrigerated until analysis.

The extraction protocol for wastewater samples was as described above, except that the blowdown process was interrupted when the extracts volume was approximately $1 \mathrm{~mL}$ when the extract was passed through a Bond Elut Carbon, 100mg, 1mL 100/pk cartridge, from Agilent technologies (USA), previously conditioned with $1 \mathrm{~mL}$ of $\mathrm{MeOH}$. The resulting extract was then blown down to $80 \mu \mathrm{L}$ and the process continued as described above.

To avoid possible adsorption of PFASs to the glassware materials, the material used for the storage was polyethylene, and the material for manipulation and extraction procedures was made of polystyrene.

\section{LC-MS/MS}

PPCPs and pesticides were analysed via LC-MS/MS, using a Shimadzu Nexera X2 HPLC system (Shimadzu Corp., Kyoto, Japan), equipped with a Kinetex® EVO C18 precolumn (5 $\mu \mathrm{m} 100 \AA$ A, LC Column 50 x 4.6 mm, Phenomenex) and a Kinetex ${ }^{\circledR}$ Biphenyl column $(2.6 \mu \mathrm{m} 100 \AA$ A, LC Column 50 x $2.1 \mathrm{~mm}$, Phenomenex). The HPLC was coupled to a Sciex QTRAP 6500+ mass spectrometer (Sciex, Concord, Ontario, Canada) equipped with electrospray ionization (ESI) (TurboV) interface (Curtain gas 30 psi, ion-spray voltage $5.4 \mathrm{kV}$, temperature $480^{\circ} \mathrm{C}$, and the ion source gases 1 and 2 at $60 \mathrm{psi}$ ). It was operated in negative and positive modes, with fast polarity switching, and multiple reaction monitoring mode (MRM), with a turbo Spray IonDrive. The mobile phases employed were (A) $\mathrm{H}_{2} \mathrm{O} 1 \% \mathrm{MeOH}, 0.1 \%$ acetic acid and (B) $\mathrm{MeOH}$ $5 \% \mathrm{H}_{2} \mathrm{O}, 0.1 \%$ Acetic acid. The linear gradient was as follows: $0 \mathrm{~min}(95 \% \mathrm{~A}), 0.5 \mathrm{~min}(90 \% \mathrm{~A}), 5.2 \mathrm{~min}$ $(0 \% \mathrm{~A}), 9.6 \mathrm{~min}(0 \% \mathrm{~A})$ and $9.61 \mathrm{~min}(95 \% \mathrm{~A})$. The injection volume was $5 \mu 1$ and column temperature was held at $45^{\circ} \mathrm{C}$. 
PFASs were analysed by UHPLC-MS/MS, using a Sciex ExionLC UHPLC system (Sciex, Concord,

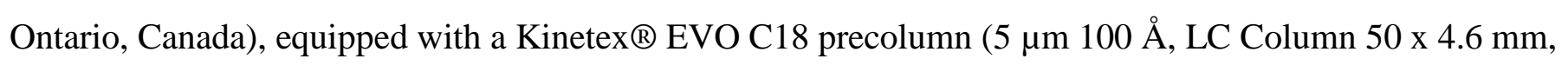
Phenomenex) and a Kinetex® EVO C18 column (2.6 ${ }^{\circledR}$ m $100 \AA$ A , LC Column 100 x 2.1 mm, Phenomenex). The mass spectrometer was a Sciex TRIPLE QUAD 6500+ (Sciex, Concord, Ontario, Canada) equipped with electrospray ionization (ESI) (TurboV), operated in negative mode and MRM, with a turbo Spray IonDrive (curtain gas $30 \mathrm{psi}$, ion-spray voltage floating at $-4.5 \mathrm{kV}$, temperature $360{ }^{\circ} \mathrm{C}$, and the ion source gas 1 and 2 at 55 psi). The mobile phases were (A) $\mathrm{H}_{2} \mathrm{O} 1 \% \mathrm{MeOH}, 8 \mathrm{mM}$ ammonium acetate, $5 \mathrm{mM} \mathrm{N}$ methyl piperidine and (B) $\mathrm{MeOH} 5 \% \mathrm{H}_{2} \mathrm{O}, 8 \mathrm{mM}$ ammonium acetate, $5 \mathrm{mM}$-methyl piperidine. The linear gradient was as follows: $0 \mathrm{~min}(85 \% \mathrm{~A}), 0.5 \mathrm{~min}(60 \% \mathrm{~A}), 6 \mathrm{~min}(0 \% \mathrm{~A}), 9 \mathrm{~min}(0 \% \mathrm{~A})$ and $9.10 \mathrm{~min}(85 \%$ A). Injection volume was $5 \mu \mathrm{l}$ and column temperature was held at $50^{\circ} \mathrm{C}$.

The identification and confirmation of compounds was made by comparing their retention times (RT) and mass spectral transitions with those from reference standards (Tables $\mathbf{S - 2}, \mathbf{S}-\mathbf{5}$ and $\mathbf{S}-\mathbf{6}$ ). The software used was MultiQuant 3.0.2 from Sciex.

\section{Text S-3. Quality assurance}

Each batch of a maximum of 10 samples included a procedural blank (MilliQ water). In addition, a "side spike" was included in every batch, made by repeating every pippeting applied to the samples, but into an empty vial. Later a MeOH: MilliQ water solution was added to obtain the same final proportion as in the extracted samples. This vial contained all the reagents present in the extracts but omitted the extraction procedure to check if the pipetting protocols and practices during the extraction procedure were correct. A full set of calibration standards ( 8 for PPCPs and pesticides, and 10 for PFASs) was injected at the beginning and the end of each analytical sequence. Also, a standard was injected every 10 samples to check for instrumental variation. Only regression coefficients $\left(\mathrm{R}^{2}\right)>0.99$ were accepted for the calibration curve. Instrumental limits of detection (ILODs) were determined as three times the standard deviation (SD) of the signal from repeated injection (7x) of a low-level standard $(0.4 \mathrm{ng} / \mathrm{mL}$ for PFASs and $0.5 \mathrm{ng} / \mathrm{mL}$ for PPCPs and pesticides). Instrumental limits of quantification (ILOQs) were set as three times the ILODs. Limits of reporting (LORs) were set to three times the average concentration in the MilliQ blanks. Any concentration below the LORs or ILODs was reported as not detected (n.d.). Repeatability was calculated as the SD divided by mean $(\% \mathrm{CV})$ of seven injections of the low-level standard. 


\section{Text S-4. Fitting models with variable reaction rate constants}

Two models with variable reaction rate constants $k_{r}$ were applied to fit the observed data for some T-B chemicals. One, applicable to chemicals removed predominantly via phototransformation, assumes a rate constant proportional to UV radiation intensity. We summed the hourly UV Index readings for Brisbane for each day during 2017 (denoted $U V_{24}$ ) and fitted them with a sin function (Fig. S-6), obtaining the equation:

$$
U V_{24}=37.503(1-0.532 \sin (t-81.6))
$$

where $t$ is calendar day. We then defined a pseudo first order rate constant for phototransformation, $k_{r p}$, with a reference state of $U_{24}=24$ (or an average UV index of 1 over a $24 \mathrm{~h}$ period):

$$
k_{r}=k_{r p} \frac{37.503}{24}(1-0.532 \sin (t-81.6))
$$

Substituting this into Eq. S-3,

$$
\frac{d\left(V C_{w}\right)}{d t}=E-G_{o u t} C_{w}-k_{r} V C_{w}
$$

and neglecting loss by advection yields:

$$
\frac{d C_{w}}{d t}=\frac{E}{V}-k_{r p} \frac{37.503}{24}(1.563-0.831 \sin (t-81.6)) C_{w}
$$

The second model with a variable reaction rate constant $k_{r}$ accounts for temperature dependent transformation using an Arrhenius-type approach. First, water temperature in the Brisbane River at Savage's Crossing during 2017 was plotted and fitted with a sin function (Fig. S-6), yielding the equation:

$$
T_{C}=23.36(1-0.236 \sin (t-111))+273
$$

where $T_{C}$ is water temperature $(\mathrm{K})$. We then defined a pseudo first order reaction rate constant at a reference state of $298 \mathrm{~K}\left(k_{r T}\right)$ and described the temperature dependence using the Arrhenius equation:

$$
k_{r}=k_{r T} \cdot e^{\left(-\frac{U}{R}\left(\frac{1}{[(23.36(1-0.236 \sin (t-111)))+273]}-\frac{1}{298}\right)\right)}
$$

where $U$ is the activation energy $(\mathrm{J} / \mathrm{mol})$ and $R$ is the gas constant $(8.314 \mathrm{~J} / \mathrm{K} \mathrm{mol})$. Substituting this into Eq. S-1, and neglecting loss by advection yields: 


$$
\frac{d C_{w}}{d t}=\frac{E}{V}-k_{r T} e^{\left(-\frac{U}{R}\left(\frac{1}{[(23.36(1-0.236 \sin (t-111)))+273]}-\frac{1}{298}\right)\right)} C_{w}
$$

For T-C chemicals that were primarily released during the flood and whose concentration tended to decrease with time thereafter, an analogous approach was employed. Substituting Eq. S-2 and Eq. S-6 into Eq. S-8 that describes transformation loss but no advective loss and no emissions post-flood.

$$
\frac{d C_{w}}{d t}=-k_{r} C_{w}
$$

affords

$$
\frac{d C_{w}}{d t}=-k_{r p}(1.563-0.831 \sin (t-81.6)) C_{w}
$$

and

$$
\frac{d C_{w}}{d t}=-k_{r T} e^{\left(-\frac{U}{R}\left(\frac{1}{[(23.36(1-0.236 \sin (t-111)))+273]}-\frac{1}{298}\right)\right)} C_{w}
$$

\section{Text S-5. Hydrological and meteorological considerations}

The Savage's Crossing Alert weather station, located approximately $60 \mathrm{~km}$ upstream of the sampling point, registered $183 \mathrm{~mm}$ of precipitation the $30^{\text {th }}$ of March [5], while stations in southern areas registered from 242 to $297 \mathrm{~mm}$ the next day [6]. Major flood levels were recorded in Ipswich, while downstream at the Brisbane river the levels remained below the threshold for a minor flood [6]. The water level at Moggill increased by about 2.5 meters during March 31. This suggests that most of the water from this event came from Ipswich through the Bremer River and then discharged into the Brisbane River just upstream of the Moggill gauging station. 


\section{Text S-6. Interpretation of temporal concentration profiles}

\section{T-A chemicals}

Compounds belonging to this group were mainly PFASs (PFPeA, PFHxA PFOA, PFDA, PFBS, PFPeS, PFHxS, PFHpS and PFOS) but also included the pesticide simazine and the pesticide metabolite desisopropyl atrazine (Fig. S-2). The concentration profiles of perfluorinated carboxylates (PFCAs) PFPeA, PFHxA PFOA and PFDA have already been discussed in the manuscript.

In contrast to the PFCAs, the perfluorosulfonates (PFSAs) PFBS, PFPeS, PFHpS and PFOS were not present in the WWTP effluent above the LOQ (Tables S-8 and S-9), and while PFHxS was detected in the WWTP effluent, these emissions were insufficient to explain the concentrations detected in the estuarine water. This indicates that there must have been other major sources of the PFSAs. Furthermore, the curvilinear time trend in $C_{W}$ exhibited by several PFSAs during the dry season followed by a sharp increase in November and December contrasts with the linear $C_{W}$ time trends shown by most PFCAs and suggests that there must have been variable emissions. High levels of PFOS+PFHxS have been detected in water and fish in the Bremer River [7] (Fig. 1). Based on their transect of PFASs contamination along the Brisbane River, Anim et al. [8,9] suggest that the Bremer River is an important source of several PFSAs to the estuary. A governmental investigation of the consequences of the use of aqueous film forming firefighting foam at the Royal Australian Air Force Base Amberley, which is located adjacent to the Bremer River, has indicated widespread off-base contamination of surface and ground water with PFASs [10]. This may be the primary source of PFSAs to the estuary. A close connection between precipitation and PFSA concentration increase in surface water [11], probably due to transport via ground and surface water to the Bremer River, could explain the observed time trend in $C_{W}$, with decreasing rates of increase of $C_{W}$ as the dry period progresses due to diminishing groundwater inflow and higher rates of increase in $C_{W}$ with the onset of the wet period in mid-October. Furthermore, the tendency of PFOS to sorb to sediments $[12,13]$ may explain its significantly more pronounced curvilinearity during the dry season, compared to the other PFSAs.

Modelling of the concentration trends of desisopropyl atrazine and simazine showed that like most of the PFCAs, the $95 \%$ confidence interval of the exponential term $\left(G_{o u t} / V+k_{r}\right)$ also intersected zero (Table 1), indicating that these two substances were very persistent in the estuary. Unlike the PFCAs, they were not present in the WWTP effluent above the LOQ (Tables S-8 and S-9). They are pesticides that could be entering the estuary through atmospheric deposition [14], drainage channels from agricultural fields [15], or groundwater discharge. The apparent constancy of the inputs during this dry period would seem to exclude the first two possibilities, making groundwater discharge the most likely source. Desisopropyl atrazine is an environmental transformation product of atrazine, sebuthylazine, and simazine, and could have been formed in the estuary after discharge of atrazine herbicides. However, the levels of atrazine and simazine in the 
WWTP effluent (n.d. and $<16 \mathrm{mg} / \mathrm{d}$, respectively) themselves are insufficient to explain the levels of desisopropyl atrazine in the estuary and there are likely to be other sources for atrazine or the other potential precursors.

\section{T-B chemicals}

Hydrochlorothiazide, gabapentin, cotinine, hydroxycotinine, diuron, carbamazepine, tramadol, desmethyl diazepam, venlafaxine, temazepam and iopromide concentrations showed a T-B time trend.

Diuron is a broad-spectrum residual herbicide. The very good agreement between estimated input flux and measured WWTP emissions (ratio of 0.97) indicates that Goodna WWTP was the main source of diuron to the estuarine segment. Since the modelled data were collected during a dry period, inputs from surface runoff are expected to have been negligible. In contrast to simazine and desisopropyl atrazine, diuron inputs from groundwater appear to have been insignificant. Indeed, Prichard et al. suggest that diuron is more likely to remain in the upper soil layers rather than infiltrate [16]. Our estimated half-life (66 d) was in the range of those measured in a laboratory biotransformation experiment using sediment-water suspensions (9-101 d) [17]. Relatively slow phototransformation (half-life of approximately 4 months) of diuron in demineralized water has been reported during exposure to natural sunlight at higher latitudes $\left(57^{\circ} \mathrm{N}\right)$ in summer [18]. Hence both biotransformation and phototransformation of diuron are possible under environmental conditions. Although our results suggested that phototransformation was the dominant transformation process (Supplementary 2), this conclusion is speculative because the model's fit was strongly influenced by the last data point. Diuron transformation in the Brisbane River may well be due to a combination of processes.

Desmethyl diazepam, also known as nordazepam, is a pharmaceutical used to treat anxiety disorders as well as a diazepam metabolite. It also shows good agreement between the modelled inputs and measured WWTP emissions (ratio of 1.32) (Table 2 and S-13). The modelled half-life (140 d) is among the longest of the chemicals studied. The UV- and temperature-dependent models showed similar AIC scores. The UVdependent model was chosen since desmethyl diazepam is known to be susceptible to phototransformation, with half-lives in the laboratory of 2-8 d [8]. The much longer half-lives in our study may be a consequence of the turbidity (40 to 100 NTU) and depth of this stretch of the Brisbane River [19], which would reduce the rate of phototransformation compared to that from the clean water and short light path length used in the laboratory study.

Hydrochlorothiazide, a diuretic, shows somewhat poorer agreement between the modelled and measured emissions (ratio of $1.8(1.2-2.3,95 \% \mathrm{CI})$ ). Given that the day-to-day variation in measured emissions was low and use is not expected to vary from week-to-week, we have no explanation for this. The half-life 
was the shortest of all T-B chemicals (18 d), and the fit to the phototransformation model was clearly the best (Table 2 and S-13). This is in accordance with the study of Zou et al [20], which reported a significant seasonality in the persistence of hydrochlorothiazide in a Swedish lake (2-49 d) and attributed this to the seasonal variation of solar radiation.

The antidepressant carbamazepine and the benzodiazepine temazepam, used for sleep disorders, both displayed good agreement between modelled and measured emissions from the WWTP (ratios of 1.03 and 0.75 respectively). The constant $k_{r}$ model provided a comparatively good fit for carbamazepine (69 d), while the UV-dependent model gave a better fit for temazepam (100 d). Temazepam and carbamazepine have both been shown to be susceptible to phototransformation in the laboratory [21, 22]. However, the attenuation of carbamazepine in natural water bodies has been reported to be very slow [23], and it has been used as a conservative tracer $[20,24]$.

The measured emissions of the contrast medium iopromide were $60 \%$ higher than the modelled emissions (Table 2 and S-13). This may be due to higher uncertainty in the measured emissions. There was relatively high day-to-day variation in measured emissions (Supplementary 2) and usage of iopromide is expected to vary more than for many of the other PPCPs studied. The UV-dependent model provided the best fit of the data. Iopromide is susceptible to phototransformation under environmental conditions [25] and has also been shown to biotransform in simulation tests with river water [26]. Therefore, its transformation may be the consequence of multiple processes. The estimated half-life in the Brisbane River (61 d) lies in the range of that observed in these simulation tests (8-69 d).

Tramadol and venlafaxine are used as an analgesic and antidepressant respectively, but are structurally similar [27]. The estimated input rate of venlafaxine was over 4 times smaller than the actual emission rate from the Goodna WWTP, while no comparison was possible for tramadol because it was not detected in the WWTP effluent. Gulde et al. [28] showed protozoa sequestered aliphatic amines (including venlafaxine and tramadol) into acid vesicles in protozoa via ion trapping. This pool may not have been accessed by our protocol, either because the sequestering organisms settle in the estuary (being not in the samples), or because the organisms are not completely extracted in the SPE column. This could explain part of the lower modelled input rate of venlafaxine compared to the WWTP emission rate. An alternative explanation is that the venlafaxine measured in water consisted primarily of ion-trapped residues, and that the freely dissolved fraction had already been largely degraded. Due to the uncertainty surrounding the nature of the residues of these weak bases in the water samples, an accurate interpretation of the observed half-lives of tramadol (69 d) and venlafaxine (72 d) is not possible.

The modelled input rate of gabapentin to the estuarine section was 260 times smaller than the measured emission rate from the WWTP (Table 2 and S-13). Further assessment showed that the measured emission 
rate of this analgesic and anticonvulsant was higher than could be expected from therapeutic use by the population serviced by the Goodna WWTP. Furthermore, there were very large fluctuations in the measured emissions over the 7-day sampling period (Table S-9) that are inconsistent with the expected use of the compound. The constant $k_{r}$ model provided a good fit of the observed concentrations in the Brisbane River, yielding a half-life of $40 \mathrm{~d}$. This relatively long half-life is difficult to reconcile with the much lower than expected concentrations in the estuary. One possible explanation for these inconsistencies is poor performance of the analytical method. More investigation is required before conclusions can be drawn about the behaviour of gabapentin in this system.

Cotinine and hydroxycotinine are nicotine metabolites. The modelled input of cotinine is 3 times greater than the measured emissions from the Goodna WWTP. This suggests that cotinine is being formed in the estuary or there are other sources. The effective absence of nicotine in the estuarine water and WWTP effluent samples (Table S-9) suggests that this chemical is not being formed from nicotine discharged by the WWTP. On the other hand, hydroxycotinine showed the opposite behaviour, with a relatively good agreement and measured emissions 30\% higher than modelled. Although a previous study conducted in southeast Queensland, Australia between 2010-2017, showed inputs of hydroxycotinine to WWTPs significantly higher than for cotinine (as in the present study), it also showed high variability of hydroxycotinine inputs [29]. Furthermore, humans excrete a large fraction of cotinine and hydroxycotinine as conjugates [30], which may uncouple once in the estuary. These could help explain the disagreement. With estimated halflives of 210 and $46 \mathrm{~d}$ respectively for cotinine and hydroxycotinine, cotinine was the most persistent T-B compound. The UV- and temperature-dependent models performed comparably for cotinine, while there was no model that performed significantly better for hydroxycotinine. Relevant literature information about the transformation processes in water was not found for hydroxycotinine and scarce for cotinine. Neither photolysis nor hydrolysis are expected to be important transformation processes for cotinine [31], while it can be biotransformed in sediment [32].

\section{T-C chemicals}

This group comprised the pesticides 2,4-D, diazinon, dicamba, methomyl, tebuthiuron and prometryn.

Methomyl displayed a highly significant exponential decrease in concentration up until mid-October when a rainy period began $\left(r^{2}=0.94\right)$ (Fig. 2). The models using UV-radiation and temperature dependent $\mathrm{k}_{\mathrm{r}}$ performed similarly, and they were clearly better than the model assuming constant $\mathrm{k}_{\mathrm{r}}$ (Supplementary 2). Methomyl has been reported to be susceptible to biotransformation and indirect photolysis, whereby the former is believed to be more rapid [33]. However, the estimated half-life (18 d) from this work was longer than that reported in laboratory biotransformation experiments using river-derived microbial biofilms (0.69 d) [34]. 
Tebuthiuron, prometryn and diazinon levels also showed a decrease over the whole course of the sampling period. The constant $\mathrm{k}_{\mathrm{r}}$ model gave the best fit to the data for tebuthiuron, and the half-life was $140 \mathrm{~d}$ (Supplementary 2). Tebuthiuron is reported to undergo slow volatilization, hydrolysis, biotransformation and phototransformation in water [35], which is consistent with the relatively long half-life we derived. In a partially shaded outdoor experiment with seawater and intertidal sediments conducted in Australia, a dissipation half-life of $944 \mathrm{~d}$ was measured [36]. It remains unclear what the primary transformation mechanisms are for tebuthiuron.

The concentration of prometryn also decreased relatively slowly over the study period. The temperature dependant $\mathrm{k}_{\mathrm{r}}$ model clearly provided the best fit to the observations, yielding an estimated half-life of $32 \mathrm{~d}$ (Supplementary 2). Biotransformation of prometryn has been widely studied [37, 38]. Liu et al. reported half-lives of few hours in wastewater [37], which is much more rapid than what we derived or the Brisbane estuary. This provides an indication of the limitations in applying biotransformation rates from laboratory studies with wastewater to the natural environment.

Diazinon is subject to hydrolysis with a reported half-life of $138 \mathrm{~d}$ at $\mathrm{pH} 7[39,40]$. This compares well with the model fit $\left(\mathrm{C}^{+}\right)$and half-life estimated for the Brisbane River (260 d), especially considering the length of the half-life and the uncertainty in our determination (95\% CI, 200-410 d).

The concentrations of 2,4-D and dicamba decreased only during part of the sampling period (Fig. S-4), and just a portion of the overall time trend data was used for the model fitting (Supplementary 2). The concentration of dicamba decreased until early June, after which it fluctuated between 55-130 ng/L. This indicates that there were ongoing inputs of this compound. No model provided a fit that was clearly better than the other models (Supplementary 2). The half-lives obtained from the period up to early June were 46 $\mathrm{d}$ for the UV-radiation dependent model and $11 \mathrm{~d}$ for the temperature dependant model. The later agrees with the half-life of 10-14 d reported by Degenhardt et al. [41] for dicamba in 2 wetlands. However, the authors in that study attributed an indeterminant portion of the attenuation in the wetlands to water infiltration, in which case the half-lives due to dicamba transformation would have been longer and therefore might have a better agreement with the half-life provided by the constant $k_{r}$ or UV-dependant model.

In contrast to Dicamba, the time trend for 2,4-D was characterized by an abrupt increase by a factor of eight in mid-September followed by an equally abrupt decrease at the end of the month (Fig. S-4). This concentration pulse could be due to new inputs from seasonal use of the pesticide. The existence of such inputs is also suggested by a more gradual increase in concentrations during June. The abrupt decrease in September would suggest rapid transformation with a half-life $<2 \mathrm{~d}$. This decrease is incompatible with the half-life of $27 \mathrm{~d}$ suggested by the exponential decrease in concentration between early April and late May (Supplementary 2). An attenuation half-life of $12 \mathrm{~d}$ was reported for 2,4-D in the same study as mentioned 
above for dicamba [41]. This $12 \mathrm{~d}$ half-life is more consistent with the $27 \mathrm{~d}$ half-life derived from the first 6 weeks of our observations, suggesting that the two high concentrations measured in September may have been the result of some anomaly. This example of 2,4-D illustrates some of the limitations to interpreting field data on chemical attenuation.

\section{Inconsistent Trends}

The compounds PFBA, haloxyfop, caffeine, imidacloprid, metalaxyl, metolachlor, imazethapyr, MCPA, desethyl atrazine, DEET, ametryn hydroxy, atrazine and atenolol displayed unstable or inconsistent temporal concentration trends. The observed trends could be due to, for example, fluctuating emissions from the WWTP, as observed for imidacloprid and metolachlor (Table S-9), or contributions from other sources. Metalaxyl and imazethapyr were not detected in Goodna WWTP effluent (Table S-9). In addition, their concentrations in the surface water were also very low, close to the LOQ (Table S-11). Hence, source attribution is not possible.

PFBA showed a remarkable concentration increase during the day just after the rain event (Table S-10). Its profile during the dry period was also quite inconsistent compared with those from the other PFASs, especially in early April. Baddiley et al. [11] also reported concentration variation along time for PFBA in the Bremer River, entering the Brisbane River estuary. However, the reasons of this inconsistency remain unknown. On the other hand, its WWTP effluent discharge was more or less stable (Table S-8). The development of a single method that works for the extraction of all PFASs is challenging, and PFBA is known to be a problematic compound when SPE is applied. The inconsistent results may have also been a result of poorer method precision or accuracy.

Caffeine, a broadly consumed stimulant, showed relatively constant WWTP emissions (Table S-9). However, there was no consistent temporal trend in the estuarine water concentration. Further research is needed to elucidate caffeine's behaviour.

Atenolol, a beta blocker, would also not be expected to exhibit variable inputs on a time scale of weeks or months. The WWTP effluent data show stable emissions on a daily time scale (Table S-9). This expected low variability in inputs to the estuary is not reflected in the time trend in water concentrations (Fig. S-2). Marked increases in early and late May are notable. Further research is needed to elucidate the reasons of this behaviour.

The herbicide metolachlor showed a very erratic time trend during early April. The concentrations during the week following the end of the flood show a significant increase followed by a rapid decrease (Table S-11). There was no clear time trend during the dry period, with concentrations ranging from 50-150 ng/L. Consequently, half-life estimation was not possible. 
The pesticides MCPA, DEET, imidacloprid, haloxyfop and atrazine showed more or less defined profiles (Fig. S-2), but these were not consistent with the mass balance model assumptions. These compounds show a significant increase during spring, which is consistent with their seasonal use as pesticides. Furthermore, they showed fluctuations in their concentration before this season (specially atrazine), which suggests that emissions were not constant.

The pesticide metabolites desethyl atrazine and ametryn hydroxy displayed a similar behaviour, especially during early April. After the rainfall event their concentrations rose rapidly, reaching an apparent steady state in less than a week (Fig. S-5). Since this transition occurred during the transition from a wet to a dry period, it is unclear if it is the result of a transition from high to negligible emissions with little transformation or significant constant emissions with rapid transformation. These two substances are metabolites, so their rate of input might be related to the concentration of their precursor compounds. However, while the concentration of atrazine did show an increase during rainfall, ametryn was not detected (Table S-11).

The concentrations of the pesticides imazethapyr and metalaxyl increased during the flood, which could indicate input via runoff (Figure S-5). These substances were not detected in the WWTP effluent. Imazethapyr concentrations fluctuated strongly from July onwards in a manner that we could not interpret. The metalaxyl concentration decreased sharply just after the rainfall period. However, most of the decrease in the concentration happened before April 7, i.e., during the time period when the estuary was still influenced by the flood. Hence this decrease could have been influenced by dilution. Afterwards the concentration remained more or less stable around $0.25-1.00 \mathrm{ng} / \mathrm{mL}$. It is unclear whether this is the result of very low inputs and slow transformation, or relatively constant ongoing inputs accompanied by relatively rapid transformation. 
Table S-1. Sample collection dates and times.

\begin{tabular}{|c|c|c|}
\hline Sampling Day & Sampling time & notes \\
\hline 29-Mar-20 & $18: 00$ & pre-rain \\
\hline 31-Mar-17 & 9:00 & - \\
\hline 02-Apr-17 & $11: 00$ & - \\
\hline 03-Apr-17 & 9:00 & - \\
\hline 04-Apr-17 & 9:00 & - \\
\hline 05-Apr-17 & 9:00 & - \\
\hline 06-Apr-17 & 9:00 & - \\
\hline 07-Apr-17 & 9:00 & - \\
\hline 09-Apr-17 & 9:00 & - \\
\hline 10-Apr-17 & 9:00 & - \\
\hline 12-Apr-17 & 9:00 & - \\
\hline 14-Apr-17 & $11: 00$ & - \\
\hline 15-Apr-17 & $10: 00$ & - \\
\hline 17-Apr-17 & $8: 00$ & - \\
\hline 19-Apr-17 & 9:00 & - \\
\hline 26-Apr-17 & 9:00 & - \\
\hline 03-May-17 & 9:00 & - \\
\hline 11-May-17 & $10: 00$ & - \\
\hline 17-May-17 & 9:00 & - \\
\hline 24-May-17 & 9:00 & - \\
\hline 31-May-17 & $1: 30$ & - \\
\hline 07-Jun-17 & - & - \\
\hline 21-Jun-17 & 9:00 & - \\
\hline 29-Jun-17 & $17: 00$ & - \\
\hline 05-Jul-17 & 9:00 & - \\
\hline 19-Jul-17 & 9:00 & - \\
\hline 27-Jul-17 & $17: 00$ & - \\
\hline 02-Aug-17 & $17: 00$ & - \\
\hline 30-Aug-17 & 9:00 & - \\
\hline 17-Sep-17 & $17: 00$ & - \\
\hline 27-Sep-17 & $17: 00$ & - \\
\hline 04-Oct-17 & 9:00 & - \\
\hline 18-Oct-17 & 9:00 & - \\
\hline 01-Nov-17 & 9:00 & - \\
\hline 16-Nov-17 & $17: 00$ & - \\
\hline 12-Dec-17 & 9:00 & - \\
\hline
\end{tabular}


Table S-2. MS and quality assurance information for PFASs.

\begin{tabular}{|c|c|c|c|c|c|c|c|c|c|}
\hline Analyte ID & $\begin{array}{r}\text { Retention Time } \\
\text { (Min) }\end{array}$ & $\begin{array}{r}\text { Q1 Mass } \\
\text { (Da) }\end{array}$ & $\begin{array}{r}\text { Q3 Mass } \\
\text { (Da) }\end{array}$ & Mass labelled & $\begin{array}{r}\text { ILOD } \\
\text { (ng/mL) }\end{array}$ & $\begin{array}{r}\text { ILOQ } \\
\text { (ng/mL) }\end{array}$ & $\begin{array}{r}\text { LOR } \\
\text { (ng/mL) }\end{array}$ & $\begin{array}{r}\text { MilliQ blank } \\
\text { (ng/mL) }\end{array}$ & $\begin{array}{r}\text { Repeatability } \\
(\% \mathrm{CV})\end{array}$ \\
\hline PFBA 1 & 1.6 & 212.8 & 169 & MPFBA & 0.03 & 0.10 & 0.16 & 0.07 & 3 \\
\hline PFPeA 1 & 3 & 262.8 & 219 & MPFPeA & 0.04 & 0.11 & 0.10 & 0.05 & 3 \\
\hline PFPeA 2 & 3 & 262.8 & 69 & MPFPeA & & & & & \\
\hline PFHXA 1 & 3.7 & 312.8 & 269 & MPFHXA & 0.06 & 0.19 & 0.12 & 0.05 & 5 \\
\hline PFHXA 2 & 3.7 & 312.8 & 119 & MPFHxA & & & & & \\
\hline PFHpA 1 & 4.27 & 362.8 & 319 & MPFHpA & 0.08 & 0.23 & 6.59 & 2.93 & 7 \\
\hline PFHpA 2 & 4.27 & 362.8 & 169 & MPFHpA & & & & & \\
\hline PFOA 1 & 4.8 & 412.8 & 369 & MPFOA & 0.09 & 0.27 & 0.28 & 0.12 & 6 \\
\hline PFOA 2 & 4.8 & 412.8 & 169 & MPFOA & & & & & \\
\hline PFOA 3 & 4.8 & 412.8 & 219 & MPFOA & & & & & \\
\hline PFNA 1 & 5.24 & 462.8 & 419 & MPFNA & 0.08 & 0.23 & 0.51 & 0.23 & 5 \\
\hline PFNA 2 & 5.24 & 462.8 & 169 & MPFNA & & & & & \\
\hline PFDA 1 & 5.7 & 512.8 & 469 & MPFDA & 0.06 & 0.18 & 0.10 & 0.04 & 5 \\
\hline PFDA 2 & 5.7 & 512.8 & 269 & MPFDA & & & & & \\
\hline PFUnDA 1 & 6 & 562.8 & 519 & MPFUnDA & 0.06 & 0.17 & 0.10 & 0.05 & 4 \\
\hline PFUnDA 2 & 6 & 562.8 & 269 & MPFUnDA & & & & & \\
\hline PFDoDA 1 & 6.3 & 612.8 & 569 & MPFDoDA & 0.13 & 0.39 & 0.07 & 0.03 & 9 \\
\hline PFDoDA 2 & 6.3 & 612.8 & 169 & MPFDoDA & & & & & \\
\hline PFTrDA 1 & 6.6 & 662.8 & 619 & M2 PFTeDA & 0.31 & 0.93 & 0.03 & 0.01 & 19 \\
\hline PFTrDA 2 & 6.6 & 662.8 & 169 & M2 PFTeDA & & & & & \\
\hline PFTeDA 1 & 6.71 & 712.8 & 669 & M2 PFTeDA & 0.23 & 0.70 & 0.03 & 0.01 & 16 \\
\hline PFTeDA 2 & 6.71 & 712.8 & 169 & M2 PFTeDA & & & & & \\
\hline PFHxDA 1 & 7.2 & 812.8 & 769 & M2 PFHxDA & 1.33 & 3.98 & 1.13 & 0.50 & 72 \\
\hline PFHxDA 2 & 7.2 & 812.8 & 169 & M2 PFHxDA & & & & & \\
\hline PFODA 1 & 7.5 & 912.8 & 869 & M2 PFHxDA & 0.42 & 1.25 & 0.12 & 0.05 & 19 \\
\hline PFODA 2 & 7.5 & 912.8 & 169 & M2 PFHxDA & & & & & \\
\hline PFBS 1 & 3.1 & 298.9 & 80 & M3 PFBS & 0.03 & 0.08 & 0.40 & 0.18 & 2 \\
\hline PFBS 2 & 3.1 & 298.9 & 99 & M3 PFBS & & & & & \\
\hline PFPeS 1 & 3.8 & 349 & 80 & M3 PFBS & 0.06 & 0.19 & 0.03 & 0.01 & 5 \\
\hline PFPeS 2 & 3.8 & 349 & 99 & M3 PFBS & & & & & \\
\hline PFHXS 1 & 4.4 & 398.8 & 80 & MPFHxS & 0.05 & 0.14 & 0.16 & 0.07 & 4 \\
\hline PFHxS 2 & 4.4 & 398.8 & 99 & MPFHxS & & & & & \\
\hline PFHxS 3 & 4.4 & 398.8 & 119 & MPFHxS & & & & & \\
\hline PFHxS 4 & 4.4 & 398.8 & 130 & MPFHxS & & & & & \\
\hline PFHps 1 & 4.9 & 448.8 & 80 & MPFHxS & 0.04 & 0.13 & 0.02 & 0.01 & 4 \\
\hline PFHps 2 & 4.9 & 448.8 & 99 & MPFHxS & & & & & \\
\hline PFOS 1 & 5.3 & 498.8 & 80 & MPFOS & 0.04 & 0.13 & 0.54 & 0.24 & 4 \\
\hline PFOS 2 & 5.3 & 498.8 & 99 & MPFOS & & & & & \\
\hline PFOS 3 & 5.3 & 498.8 & 169 & MPFOS & & & & & \\
\hline PFOS 4 & 5.3 & 498.8 & 230 & MPFOS & & & & & \\
\hline PFNS 1 & 5.7 & 548.8 & 80 & MPFOS & 0.09 & 0.26 & 0.00 & n.d. & 8 \\
\hline PFNS 2 & 5.7 & 548.8 & 99 & MPFOS & & & & & \\
\hline PFDS 1 & 6 & 598.8 & 80 & MPFOS & 0.10 & 0.30 & 0.04 & 0.02 & 9 \\
\hline PFDS 2 & 6 & 598.8 & 99 & MPFOS & & & & & \\
\hline PFDoDS 1 & 6.5 & 698.8 & 80 & MPFOS & 0.12 & 0.35 & 0.03 & 0.01 & 17 \\
\hline PFDoDS 2 & 6.5 & 698.8 & 99 & MPFOS & & & & & \\
\hline FOSA 1 & 6.1 & 497.8 & 78 & M8 FOSA & 0.15 & 0.44 & 0.09 & 0.04 & 12 \\
\hline
\end{tabular}




\begin{tabular}{|c|c|c|c|c|c|c|c|c|c|}
\hline Analyte ID & $\begin{array}{r}\text { Retention Time } \\
\text { (Min) }\end{array}$ & $\begin{array}{r}\text { Q1 Mass } \\
\text { (Da) }\end{array}$ & $\begin{array}{r}\text { Q3 Mass } \\
\text { (Da) }\end{array}$ & Mass labelled & $\begin{array}{r}\text { ILOD } \\
\text { (ng/mL) }\end{array}$ & $\begin{array}{r}\text { ILOQ } \\
\text { (ng/mL) }\end{array}$ & $\begin{array}{r}\text { LOR } \\
\text { (ng/mL) }\end{array}$ & $\begin{array}{r}\text { MilliQ blank } \\
\text { (ng/mL) }\end{array}$ & $\begin{array}{r}\text { Repeatability } \\
(\% \mathrm{CV})\end{array}$ \\
\hline FOSA 2 & 6.1 & 497.8 & 64 & M8 FOSA & & & & & \\
\hline FOSAA 1 & 5.5 & 556 & 169 & & 0.22 & 0.65 & 0.02 & 0.01 & 21 \\
\hline FOSAA 2 & 5.5 & 556 & 78 & & & & & & \\
\hline N-Et FOSAA 1 & 6 & 583.8 & 419 & D5 N-Et FOSAA & 0.09 & 0.28 & 0.00 & n.d. & 7 \\
\hline N-Et FOSAA 2 & 6 & 583.8 & 219 & D5 N-Et FOSAA & & & & & \\
\hline N-Me FOSAA 1 & 5.9 & 569.8 & 419 & D3 N-Me FOSAA & 0.10 & 0.30 & 0.01 & 0.00 & 8 \\
\hline N-Me FOSAA 2 & 5.9 & 569.8 & 512 & D3 N-Me FOSAA & & & & & \\
\hline N-Me FOSA 1 & 6.6 & 511.8 & 219 & D3 N-Me FOSA & 0.11 & 0.33 & 0.00 & n.d. & 10 \\
\hline N-Me FOSA 2 & 6.6 & 511.8 & 169 & D3 N-Me FOSA & & & & & \\
\hline N-Et FOSA 1 & 6.8 & 525.8 & 169 & D5 N-Et FOSA & 0.40 & 1.19 & 0.00 & n.d. & 40 \\
\hline N-Et FOSA 2 & 6.8 & 525.8 & 219 & D5 N-Et FOSA & & & & & \\
\hline 10:2 FTS 1 & 6.2 & 626.9 & 607 & M2 8:2 FTS & 0.15 & 0.44 & 0.02 & 0.01 & 13 \\
\hline 10:2 FTS 2 & 6.2 & 626.9 & 81 & M2 8:2 FTS & & & & & \\
\hline $8: 2$ FTS 1 & 5.7 & 526.8 & 507 & M2 8:2 FTS & 0.10 & 0.29 & 0.02 & 0.01 & 7 \\
\hline $8: 2$ FTS 2 & 5.7 & 526.8 & 81 & M2 8:2 FTS & & & & & \\
\hline $6: 2$ FTS 1 & 4.8 & 426.8 & 407 & M2 6:2 FTS & 0.07 & 0.22 & 0.05 & 0.02 & 5 \\
\hline 6:2 FTS 2 & 4.8 & 426.8 & 81 & M2 6:2 FTS & & & & & \\
\hline 4:2 FTS 1 & 3.6 & 326.8 & 307 & M2 4:2FTS & 0.05 & 0.14 & 0.01 & 0.01 & 4 \\
\hline $4: 2$ FTS 2 & 3.6 & 326.8 & 81 & M2 4:2FTS & & & & & \\
\hline N-Me FOSE & 6.6 & 616 & 59 & D7 N-Me FOSE & 0.62 & 1.85 & 0.59 & 0.26 & 45 \\
\hline N-Et FOSE & 6.8 & 630 & 59 & D9 N-Et FOSE & 0.20 & 0.59 & 0.38 & 0.17 & 12 \\
\hline PFECHS 1 & 4.8 & 460.9 & 381 & MPFOS & 0.04 & 0.12 & 0.01 & n.d. & 3 \\
\hline PFECHS 2 & 4.8 & 460.9 & 99 & MPFOS & & & & & \\
\hline 6:2 PAP 1 & 4.1 & 442.9 & 97 & & 0.10 & 0.30 & 0.00 & n.d. & 8 \\
\hline $6: 2$ PAP 2 & 4.1 & 442.9 & 79 & & 0.05 & 0.18 & & & \\
\hline $8: 2$ PAP 1 & 5.3 & 542.9 & 79 & & 0.48 & 1.43 & 0.00 & n.d. & 41 \\
\hline 8:2 PAP 2 & 5.3 & 542.9 & 97 & & 0.72 & 2.39 & & & \\
\hline SamPAP 1 & 5.9 & 649.9 & 525.9 & & 0.19 & 0.58 & 0.00 & n.d. & 17 \\
\hline SamPAP 2 & 5.9 & 649.9 & 79 & & 0.80 & 2.68 & & & \\
\hline 6:2 DiPAP 1 & 6.7 & 788.8 & 442.9 & & 0.12 & 0.35 & 12.44 & 5.53 & 31 \\
\hline 6:2 DiPAP 2 & 6.7 & 788.8 & 97 & & 0.03 & 0.12 & & & \\
\hline 8:2 DiPAP 1 & 7.3 & 988.7 & 542.9 & & 0.44 & 1.48 & 0.00 & n.d. & \\
\hline 8:2 DiPAP 2 & 7.3 & 988.7 & 97 & & 0.39 & 1.30 & & & \\
\hline 6:2 8:2 DiPAP 1 & 7.1 & 888.8 & 97 & & 0.10 & 0.32 & 0.00 & n.d. & \\
\hline 6:2 8:2 DiPAP 2 & 7.1 & 888.8 & 542.9 & & 0.09 & 0.31 & & & \\
\hline
\end{tabular}


Table S-3. MS information for labelled PFAS standards.

\begin{tabular}{|c|c|c|c|}
\hline Analyte ID & $\begin{array}{r}\text { Retention Time } \\
\text { (Min) }\end{array}$ & $\begin{array}{r}\text { Q1 Mass } \\
\text { (Da) }\end{array}$ & $\begin{array}{r}\text { Q3 Mass } \\
(\mathrm{Da})\end{array}$ \\
\hline MPFBA & 1.6 & 216.8 & 172 \\
\hline MPFPeA & 3 & 265.9 & 222 \\
\hline MPFHxA & 3.7 & 314.9 & 270 \\
\hline MPFHpA & 4.27 & 321.8 & 322 \\
\hline MPFOA1 & 4.79 & 416.9 & 372 \\
\hline MPFOA2 & 4.79 & 416.9 & 169 \\
\hline MPFNA & 5.3 & 467.8 & 423 \\
\hline MPFDA & 5.7 & 514.8 & 470 \\
\hline MPFUdA & 6 & 564.8 & 520 \\
\hline MPFDoA & 6.3 & 614.8 & 570 \\
\hline M2PFTeDA 1 & 6.72 & 714.8 & 670 \\
\hline M2PFTeDA 2 & 6.72 & 714.8 & 169 \\
\hline M2PFHxDA 1 & 7.2 & 814.8 & 770 \\
\hline M2PFHxDA 2 & 7.2 & 814.8 & 169 \\
\hline M3 PFBS 1 & 3.1 & 301.8 & 80 \\
\hline M3 PFBS 2 & 3.1 & 301.8 & 99 \\
\hline MPFHxS 1 & 4.4 & 402.8 & 103 \\
\hline MPFHxS 2 & 4.4 & 402.9 & 84 \\
\hline MPFOS1 & 5.25 & 502.9 & 80 \\
\hline MPFOS2 & 5.25 & 502.9 & 99 \\
\hline M8 FOSA 1 & 6 & 505.9 & 78 \\
\hline M8 FOSA 2 & 6 & 505.9 & 64 \\
\hline D5 N-Et FOSAA 1 & 6 & 588.9 & 419 \\
\hline D5 N-Et FOSAA 2 & 6 & 588.9 & 483 \\
\hline D3 N-Me FOSAA 1 & 5.78 & 572.9 & 419 \\
\hline D3 N-Me FOSAA 2 & 5.78 & 572.9 & 219 \\
\hline D3 N-Me FOSA 1 & 6.38 & 514.9 & 169 \\
\hline D3 N-Me FOSA 2 & 6.38 & 514.9 & 219 \\
\hline D5 N-Et FOSA 1 & 6.67 & 530.9 & 169 \\
\hline D5 N-Et FOSA 2 & 6.67 & 530.9 & 219 \\
\hline M2 8:2 FTS 1 & 5.7 & 528.9 & 509 \\
\hline M2 8:2 FTS 2 & 5.7 & 528.9 & 80.9 \\
\hline M2 6:2 FTS 1 & 4.8 & 428.9 & 409 \\
\hline M2 6:2 FTS 2 & 4.8 & 428.9 & 81 \\
\hline M2 4:2FTS 1 & 3.6 & 328.9 & 309 \\
\hline M2 4:2FTS 2 & 3.6 & 328.9 & 80.9 \\
\hline D7 N-Me FOSE 1 & 6.6 & 623 & 59 \\
\hline D7 N-Me FOSE 2 & 6.6 & 609 & 45 \\
\hline D9 N-Et FOSE 1 & 6.8 & 639 & 59 \\
\hline D9 N-Et FOSE 2 & 6.8 & 625 & 45 \\
\hline M2 6:2 PAP 1 & 4.01 & 444.9 & 79 \\
\hline M2 6:2 PAP 2 & 4.01 & 444.9 & 97 \\
\hline M2 8:2 PAP 1 & 5.22 & 544.9 & 79 \\
\hline M2 8:2 PAP 2 & 5.22 & 544.9 & 97 \\
\hline M4 6:2 DiPAP 1 & 6.7 & 792.8 & 445 \\
\hline M4 6:2 DiPAP 2 & 6.7 & 792.8 & 97 \\
\hline M4 8:2 DiPAP 1 & 7.3 & 992.8 & 544.9 \\
\hline M4 8:2 DiPAP 2 & 7.3 & 992.8 & 97 \\
\hline
\end{tabular}


Table S-4. MS information for PFAS Recovery standards.

\begin{tabular}{rrrr}
\hline Analyte ID & $\begin{array}{r}\text { Retention Time } \\
\text { (Min) }\end{array}$ & $\begin{array}{r}\text { Q1 Mass } \\
(\text { Da) }\end{array}$ & $\begin{array}{r}\text { Q3 Mass } \\
\text { (Da) }\end{array}$ \\
\hline M3PFBA & 1.6 & 215.9 & 172 \\
M5PFPeA 1 & 3 & 267.9 & 223 \\
M5PFPeA 2 & 3 & 267.9 & 70 \\
M8PFOA1 & 4.9 & 420.9 & 172 \\
M8PFOA 2 & 4.9 & 420.9 & 376 \\
M3MPHxS 1 & 4.33 & 401.9 & 80 \\
M3MPHxS 2 & 4.33 & 401.9 & 99 \\
M8PFOS1 & 5.3 & 506.8 & 80 \\
M8PFOS2 & 5.3 & 506.8 & 99 \\
\hline
\end{tabular}


Table S-5. MS and quality assurance information for PPCPs and pesticides analysed in ESI positive mode.

\begin{tabular}{|c|c|c|c|c|c|c|c|c|c|}
\hline Analyte ID & $\begin{array}{r}\begin{array}{r}\text { Retention time } \\
(\text { Min) }\end{array} \\
\end{array}$ & $\begin{array}{r}\text { Q1 Mass } \\
\text { (Da) }\end{array}$ & $\begin{array}{r}\text { Q3 Mass } \\
\text { (Da) }\end{array}$ & Mass labelled & $\begin{array}{r}\text { ILOD } \\
\text { (ng/mL) } \\
\end{array}$ & $\begin{array}{r}\text { ILOQ } \\
\text { (ng/mL) } \\
\end{array}$ & $\begin{array}{r}\text { LOR } \\
\text { (ng/mL) } \\
\end{array}$ & $\begin{array}{r}\text { MilliQ blank } \\
(\mathrm{ng} / \mathrm{mL})\end{array}$ & $\begin{array}{r}\begin{array}{r}\text { Repeatability } \\
(\% \mathrm{CV})\end{array} \\
\end{array}$ \\
\hline Paracetamol 1 & 1.56 & 152.1 & 110 & Paracetamol D4 & 0.37 & 1.10 & 3.04 & 1.35 & 15 \\
\hline Paracetamol 2 & 1.56 & 152.1 & 65.1 & Paracetamol D4 & & & & & \\
\hline 3,4 DiCl Aniline 1 & 5.63 & 162 & 127 & & 0.08 & 0.24 & 0.51 & 0.23 & 5 \\
\hline 3,4 DiCl Aniline 2 & 5.63 & 162 & 74 & & & & & & \\
\hline Methomyl 1 & 4.39 & 163.1 & 88.1 & Methomyl D3 & 0.05 & 0.14 & 0.00 & 0.00 & 3 \\
\hline Methomyl 2 & 4.39 & 163.1 & 106 & Methomyl D3 & & & & & \\
\hline Nicotine 1 & 0.94 & 163.1 & 132 & Nicotine D4 & & & 0.93 & 0.41 & \\
\hline Nicotine 2 & 0.94 & 163.1 & 106.1 & Nicotine D4 & & & & & \\
\hline Gabapentin 1 & 3.14 & 172.1 & 154 & Gabapentin D10 & 0.02 & 0.05 & 0.00 & n.d. & 1 \\
\hline Gabapentin 2 & 3.14 & 172.1 & 137 & Gabapentin D10 & & & & & \\
\hline Desisopropyl Atrazine 1 & 4.07 & 174 & 104 & Desisopropyl Atrazine D5 & 0.05 & 0.16 & 0.00 & n.d. & 4 \\
\hline Desisopropyl Atrazine 2 & 4.07 & 174 & 96 & Desisopropyl Atrazine D5 & & & & & \\
\hline Cotinine 1 & 3.38 & 177.1 & 80 & Cotinine D3 & 0.02 & 0.05 & 0.16 & 0.07 & 1 \\
\hline Cotinine 2 & 3.38 & 177.1 & 98 & Cotinine D3 & & & & & \\
\hline Paraxanthine 1 & 3.99 & 181 & 124 & Paraxanthine D3 & & & 0.23 & 0.10 & \\
\hline Paraxanthine 2 & 3.99 & 181.1 & 96 & Paraxanthine D3 & & & & & \\
\hline Simazine hydroxy 1 & 3.57 & 184.1 & 114 & & 0.28 & 0.85 & 0.00 & n.d. & 32 \\
\hline Simazine hydroxy 2 & 3.57 & 184.1 & 69 & & & & & & \\
\hline Desethyl Atrazine 1 & 4.81 & 188 & 146 & Desethyl Atrazine D6 & 0.01 & 0.04 & 0.08 & 0.04 & 1 \\
\hline Desethyl Atrazine 2 & 4.81 & 188 & 104 & Desethyl Atrazine D6 & & & & & \\
\hline Clopyralid 1 & 2.9 & 192 & 110 & & 0.05 & 0.15 & 0.00 & n.d. & 3 \\
\hline Clopyralid 2 & 2.9 & 192 & 146 & & & & & & \\
\hline 5HIAA 1 & 0.1 & 192.1 & 146.1 & 5HIAA D2 & & & 0.00 & n.d. & \\
\hline 5HIAA 2 & 0.1 & 192.1 & 117.1 & 5HIAA D2 & & & & & \\
\hline DEET 1 & 6.26 & 192.1 & 119 & DEET D7 & 0.04 & 0.13 & 3.04 & 1.35 & 3 \\
\hline DEET 2 & 6.26 & 192.1 & 91 & DEET D7 & & & & & \\
\hline Hydroxycotinine 1 & 1.4 & 193.1 & 134.1 & Hydroxycotinine D3 & 0.06 & 0.18 & 0.95 & 0.42 & 3 \\
\hline Hydroxycotinine 2 & 1.4 & 193.1 & 80.1 & Hydroxycotinine D3 & & & & & \\
\hline Caffeine 1 & 4.75 & 195.1 & 138.1 & Caffeine $13 C 3$ & 0.04 & 0.13 & 0.85 & 0.38 & 3 \\
\hline Caffeine 2 & 4.75 & 195.1 & 110.1 & Caffeine $13 C 3$ & & & & & \\
\hline Ametryn hydroxy 1 & 4.2 & 198.11 & 156 & & 0.32 & 0.96 & 0.05 & 0.02 & 33 \\
\hline Ametryn hydroxy 2 & 4.2 & 198.11 & 86 & & & & & & \\
\hline Pyrimethanil 1 & 6.31 & 200.1 & 107 & & 0.15 & 0.45 & 0.10 & 0.04 & 10 \\
\hline Pyrimethanil 2 & 6.31 & 200.1 & 183 & & & & & & \\
\hline Terbuthylazine desethyl 1 & 5.54 & 202 & 146 & Tebuthylazine desethyl D9 & 0.03 & 0.10 & 0.10 & 0.05 & 2 \\
\hline Terbuthylazine desethyl 2 & 5.54 & 202 & 104 & Tebuthylazine desethyl D9 & & & & & \\
\hline Terbuthylazine desethyl 3 & 5.54 & 202.1 & 110 & Tebuthylazine desethyl D9 & & & & & \\
\hline Simazine 1 & 5.55 & 202.1 & 132 & Simazine D10 & 0.10 & 0.29 & 0.03 & 0.01 & 7 \\
\hline Simazine 2 & 5.55 & 202.1 & 124 & Simazine D10 & & & & & \\
\hline DCPU 2 & 5.49 & 205.01 & 127 & & 0.08 & 0.24 & 0.00 & n.d. & 5 \\
\hline DCPU 1 & 5.49 & 205.03 & 162 & & & & & & \\
\hline Propoxur 1 & 5.74 & 210.1 & 168.1 & Propoxur D3 & 0.16 & 0.48 & 0.00 & n.d. & 10 \\
\hline Propoxur 2 & 5.74 & 210.1 & 111 & Propoxur D3 & & & & & \\
\hline Metribuzin 1 & 5.75 & 215.1 & 187 & Metribuzin D3 & 0.09 & 0.28 & 7.33 & 3.26 & 6 \\
\hline Metribuzin 2 & 5.75 & 215.1 & 84 & Metribuzin D3 & & & & & \\
\hline Atrazine 1 & 5.88 & 216.1 & 174 & Atrazine D5 & 0.04 & 0.13 & 0.00 & n.d. & 3 \\
\hline
\end{tabular}




\begin{tabular}{|c|c|c|c|c|c|c|c|c|c|}
\hline Analyte ID & $\begin{array}{r}\text { Retention time } \\
\text { (Min) }\end{array}$ & $\begin{array}{r}\text { Q1 Mass } \\
\text { (Da) }\end{array}$ & $\begin{array}{r}\text { Q3 Mass } \\
\text { (Da) }\end{array}$ & Mass labelled & $\begin{array}{r}\text { ILOD } \\
\text { (ng/mL) }\end{array}$ & $\begin{array}{r}\text { ILOQ } \\
\text { (ng/mL) }\end{array}$ & $\begin{array}{r}\text { LOR } \\
\text { (ng/mL) } \\
\end{array}$ & $\begin{array}{r}\text { MilliQ blank } \\
\text { (ng/mL) }\end{array}$ & $\begin{array}{r}\begin{array}{r}\text { Repeatability } \\
(\% \mathrm{CV})\end{array} \\
\end{array}$ \\
\hline Atrazine 2 & 5.88 & 216.1 & 96 & Atrazine D5 & & & & & \\
\hline DCPMU 1 & 5.68 & 219.01 & 127 & & 0.05 & 0.14 & 2.16 & 0.96 & 3 \\
\hline DCPMU 2 & 5.68 & 219.02 & 162 & & & & & & \\
\hline Dichlorvos 1 & 5.53 & 221 & 109 & & 0.06 & 0.17 & 0.03 & 0.01 & 4 \\
\hline Dichlorvos 2 & 5.53 & 223 & 109 & & & & & & \\
\hline Carbofuran 1 & 5.88 & 222.1 & 165.2 & Carbofuran D3 & 0.01 & 0.04 & 0.00 & n.d. & 1 \\
\hline Carbofuran 2 & 5.88 & 222.1 & 123 & Carbofuran D3 & & & & & \\
\hline Methiocarb 1 & 6.4 & 226.1 & 169.2 & Methiocarb D3 & 0.11 & 0.33 & 0.12 & 0.06 & 7 \\
\hline Methiocarb 2 & 6.4 & 226.1 & 121 & Methiocarb D3 & & & & & \\
\hline Ametryn 1 & 6.07 & 228.2 & 186 & & 0.20 & 0.61 & 0.12 & 0.05 & 16 \\
\hline Ametryn 2 & 6.07 & 228.2 & 116 & & & & & & \\
\hline Tebuthiuron 1 & 5.7 & 229.2 & 172 & & 0.06 & 0.19 & 0.00 & n.d. & 4 \\
\hline Tebuthiuron 2 & 5.7 & 229.2 & 116 & & & & & & \\
\hline Propazine 1 & 6.15 & 230 & 146 & Propazine D6 & 0.03 & 0.08 & 0.07 & 0.03 & 2 \\
\hline Propazine 2 & 6.15 & 230 & 188 & Propazine D6 & & & & & \\
\hline Terbuthylazine 1 & 6.24 & 230.1 & 174 & Terbuthylazine D5 & 0.14 & 0.41 & 0.17 & 0.07 & 10 \\
\hline Terbuthylazine 2 & 6.24 & 230.1 & 104 & Terbuthylazine D5 & & & & & \\
\hline Asulam 1 & 3.83 & 231 & 156 & & 0.04 & 0.12 & 0.00 & n.d. & 3 \\
\hline Asulam 2 & 3.83 & 231 & 108 & & & & & & \\
\hline Naproxen 1 & 6.4 & 231.2 & 185.1 & & 0.10 & 0.30 & 0.05 & 0.02 & 6 \\
\hline Naproxen 2 & 6.4 & 231.2 & 170.1 & & & & & & \\
\hline Diuron 1 & 5.88 & 233.05 & 72 & Diuron D6 & 0.12 & 0.36 & 0.30 & 0.13 & 8 \\
\hline Diuron 2 & 5.88 & 233.05 & 46 & Diuron D6 & & & & & \\
\hline Fluometuron 1 & 5.59 & 233.1 & 72 & & 0.07 & 0.22 & 0.10 & 0.04 & 5 \\
\hline Fluometuron 2 & 5.59 & 233.1 & 46 & & & & & & \\
\hline Carbamazepine 1 & 6.11 & 237.2 & 194 & Carbamazepine D10 & 0.17 & 0.51 & 0.86 & 0.38 & 8 \\
\hline Carbamazepine 2 & 6.11 & 237.2 & 193 & Carbamazepine D10 & & & & & \\
\hline Prometryn 1 & 6.31 & 242.2 & 200.1 & Prometryn D6 & 0.13 & 0.38 & 0.26 & 0.12 & 9 \\
\hline Prometryn 2 & 6.31 & 242.2 & 158 & Prometryn D6 & & & & & \\
\hline Terbutryn 1 & 6.39 & 242.2 & 91.2 & & 1.69 & 5.08 & 9.26 & 4.11 & 68 \\
\hline Terbutryn 2 & 6.39 & 242.2 & 71.1 & & & & & & \\
\hline Picloram 1 & 3.8 & 243 & 197 & & 0.11 & 0.33 & 0.00 & n.d. & 8 \\
\hline Picloram 2 & 3.8 & 243 & 143 & & & & & & \\
\hline Hexazinone 1 & 6.1 & 253.2 & 171 & Hexazinone D6 & 0.25 & 0.74 & 0.00 & n.d. & 18 \\
\hline Hexazinone 2 & 6.1 & 253.2 & 71 & Hexazinone D6 & & & & & \\
\hline Fluroxypyr 1 & 5.35 & 255 & 209 & & 0.07 & 0.22 & 0.04 & 0.02 & 5 \\
\hline Fluroxypyr 2 & 5.35 & 255 & 181 & & & & & & \\
\hline Imidacloprid 1 & 5.26 & 256.1 & 209.1 & Imidacloprid D4 & 0.04 & 0.13 & 0.14 & 0.06 & 3 \\
\hline Imidacloprid 2 & 5.26 & 256.1 & 175 & Imidacloprid D4 & & & & & \\
\hline Bromacil 1 & 5.55 & 261.2 & 205 & Bromacil D3 & 0.09 & 0.28 & 0.00 & n.d. & 5 \\
\hline Bromacil 2 & 5.55 & 263.2 & 207 & Bromacil D3 & & & & & \\
\hline Tramadol 1 & 4.6 & 264.2 & 58 & Tramadol D6 & 0.08 & 0.25 & 0.62 & 0.27 & 5 \\
\hline Tramadol 2 & 4.6 & 264.2 & 42 & Tramadol D6 & & & & & \\
\hline Atenolol 1 & 2.82 & 267.2 & 190 & Atenolol D7 & 0.03 & 0.10 & 0.05 & 0.02 & 2 \\
\hline Atenolol 2 & 2.82 & 267.2 & 145 & Atenolol D7 & & & & & \\
\hline DesmethylDiazepam 1 & 6.58 & 271.2 & 140.1 & & 0.06 & 0.17 & 0.00 & n.d. & 4 \\
\hline DesmethylDiazepam 2 & 6.58 & 271.2 & 165.1 & & & & & & \\
\hline
\end{tabular}




\begin{tabular}{|c|c|c|c|c|c|c|c|c|c|}
\hline Analyte ID & $\begin{array}{r}\text { Retention time } \\
\text { (Min) }\end{array}$ & $\begin{array}{r}\text { Q1 Mass } \\
\text { (Da) }\end{array}$ & $\begin{array}{r}\text { Q3 Mass } \\
\text { (Da) }\end{array}$ & Mass labelled & $\begin{array}{r}\text { ILOD } \\
\text { (ng/mL) } \\
\end{array}$ & $\begin{array}{r}\text { ILOQ } \\
\text { (ng/mL) }\end{array}$ & $\begin{array}{r}\text { LOR } \\
\text { (ng/mL) }\end{array}$ & $\begin{array}{r}\text { MilliQ blank } \\
\text { (ng/mL) }\end{array}$ & $\begin{array}{r}\text { Repeatability } \\
(\% \mathrm{CV}) \\
\end{array}$ \\
\hline Imazapic 1 & 5.34 & 276.1 & 231.1 & & 0.09 & 0.27 & 0.00 & n.d. & 5 \\
\hline Imazapic 2 & 5.34 & 276.1 & 163 & & & & & & \\
\hline Venlafaxine 1 & 5.05 & 278.2 & 58 & Venlafaxine D6 & 0.04 & 0.12 & 0.08 & 0.03 & 3 \\
\hline Venlafaxine 2 & 5.05 & 278.2 & 121 & Venlafaxine D6 & & & & & \\
\hline Metalaxyl 1 & 6.42 & 280.2 & 220.1 & & 0.05 & 0.16 & 0.02 & 0.01 & 3 \\
\hline Metalaxyl 2 & 6.42 & 280.2 & 192.1 & & & & & & \\
\hline Pendimethalin 1 & 7.51 & 282.1 & 212.1 & & 0.08 & 0.24 & 0.10 & 0.04 & 7 \\
\hline Pendimethalin 2 & 7.51 & 282.1 & 194.1 & & & & & & \\
\hline Metolachlor 1 & 6.87 & 284.2 & 252 & Metolachlor D6 & 0.26 & 0.79 & 0.02 & 0.01 & 18 \\
\hline Metolachlor 2 & 6.87 & 284.2 & 176 & Metolachlor D6 & & & & & \\
\hline Imazethapyr 1 & 5.72 & 290.1 & 177.1 & & 0.04 & 0.12 & 0.01 & n.d. & 3 \\
\hline Imazethapyr 2 & 5.72 & 290.1 & 106 & & & & & & \\
\hline Codeine 1 & 4.02 & 300.2 & 215.1 & Codeine D3 & 0.08 & 0.25 & 0.10 & 0.05 & 6 \\
\hline Codeine 2 & 4.02 & 300.2 & 165.1 & Codeine D3 & & & & & \\
\hline Temazepam 1 & 6.69 & 301.2 & 255.1 & Temazepam D5 & 0.09 & 0.27 & 0.15 & 0.07 & 6 \\
\hline Temazepam 2 & 6.69 & 301.2 & 283.1 & Temazepam D5 & & & & & \\
\hline Fenamiphos 1 & 6.7 & 304.15 & 217.1 & & 0.07 & 0.21 & 0.00 & n.d. & 4 \\
\hline Fenamiphos 2 & 6.7 & 304.15 & 202.1 & & & & & & \\
\hline Diazinon 1 & 6.86 & 305.3 & 169.1 & Diazinon D10 & 0.02 & 0.07 & 0.07 & 0.03 & 2 \\
\hline Diazinon 2 & 6.86 & 305.3 & 153 & Diazinon D10 & & & & & \\
\hline Tebuconazole 1 & 6.69 & 308.15 & 70 & Tebuconazole D6 & 0.05 & 0.15 & 0.02 & 0.01 & 3 \\
\hline Tebuconazole 2 & 6.69 & 310.15 & 70 & Tebuconazole D6 & & & & & \\
\hline Fluoxetine 1 & 5.55 & 310.1 & 44 & Fluoxetine D6 & 0.27 & 0.82 & 0.29 & 0.13 & 25 \\
\hline Fluoxetine 2 & 5.55 & 310.1 & 148 & Fluoxetine D6 & & & & & \\
\hline Desmethyl Citalopram 1 & 5.32 & 311.3 & 109 & & 0.04 & 0.13 & 0.00 & n.d. & 4 \\
\hline Desmethyl Citalopram 2 & 5.32 & 311.3 & 262.2 & & & & & & \\
\hline Citalopram 1 & 5.34 & 325.3 & 109 & Citalopram D6 & 0.10 & 0.31 & 0.88 & 0.39 & 5 \\
\hline Citalopram 2 & 5.34 & 325.3 & 262.2 & Citalopram D6 & & & & & \\
\hline Fluazifop 1 & 6.39 & 328.2 & 282.2 & Fluazifop D4+ & 0.06 & 0.18 & 0.04 & 0.02 & 4 \\
\hline Fluazifop 2 & 6.39 & 328.2 & 254.1 & Fluazifop D4+ & & & & & \\
\hline Malathion 1 & 6.69 & 331.1 & 127 & & 0.08 & 0.25 & 0.00 & n.d. & 9 \\
\hline Malathion 2 & 6.69 & 331.1 & 99 & & & & & & \\
\hline Propiconazole 1 & 7 & 342 & 159 & Propiconazole D5 & 0.04 & 0.12 & 0.05 & 0.02 & 3 \\
\hline Propiconazole 2 & 7 & 344 & 161 & Propiconazole D5 & & & & & \\
\hline Prothioconazole 1 & 0.1 & 344 & 154 & & & & 0.00 & n.d. & \\
\hline Prothioconazole 2 & 0.1 & 344 & 290 & & & & & & \\
\hline Chlorpyriphos 1 & 7.37 & 350.1 & 198 & & 0.08 & 0.24 & 0.02 & 0.01 & 7 \\
\hline Chlorpyriphos 2 & 7.37 & 350.1 & 97 & & & & & & \\
\hline Haloxyfop + 1 & 6.72 & 362.2 & 316.2 & Haloxyfop D4+ & 0.06 & 0.19 & 0.02 & 0.01 & 4 \\
\hline Haloxyfop + 2 & 6.72 & 362.2 & 288 & Haloxyfop D4+ & & & & & \\
\hline Metsulfuron-Methyl 1 & 6.31 & 382.1 & 167 & Metsulfuron-methyl D3 & 0.05 & 0.15 & 1.08 & 0.48 & 2 \\
\hline Metsulfuron-Methyl 2 & 6.31 & 382.1 & 199 & Metsulfuron-methyl D3 & & & & & \\
\hline Tadalafil 1 & 6.61 & 390.2 & 268.1 & & 0.10 & 0.29 & 0.00 & n.d. & 6 \\
\hline Tadalafil 2 & 6.61 & 390.2 & 204 & & & & & & \\
\hline Verapamil 1 & 5.71 & 455.2 & 165.1 & & 0.05 & 0.16 & 0.05 & 0.02 & 4 \\
\hline Verapamil 2 & 5.71 & 455.2 & 303.2 & & & & & & \\
\hline Sildenafil 1 & 5.7 & 475.2 & 58 & & 0.08 & 0.25 & 0.00 & n.d. & 7 \\
\hline
\end{tabular}




\begin{tabular}{|c|c|c|c|c|c|c|c|c|c|}
\hline Analyte ID & $\begin{array}{r}\text { Retention time } \\
\text { (Min) }\end{array}$ & $\begin{array}{r}\text { Q1 Mass } \\
(\mathrm{Da})\end{array}$ & $\begin{array}{r}\text { Q3 Mass } \\
\text { (Da) }\end{array}$ & Mass labelled & $\begin{array}{r}\text { ILOD } \\
\text { (ng/mL) } \\
\end{array}$ & $\begin{array}{r}\text { ILOQ } \\
\text { (ng/mL) }\end{array}$ & $\begin{array}{r}\text { LOR } \\
\text { (ng/mL) }\end{array}$ & $\begin{array}{r}\text { MilliQ blank } \\
\text { (ng/mL) }\end{array}$ & $\begin{array}{r}\begin{array}{r}\text { Repeatability } \\
(\% \mathrm{CV})\end{array} \\
\end{array}$ \\
\hline Sildenafil 2 & 5.7 & 475.2 & 283.1 & & & & & & \\
\hline Atorvastatin 1 & 6.8 & 559.5 & 440.3 & Atorvastatin D5 & 0.26 & 0.77 & 0.40 & 0.18 & 19 \\
\hline Atorvastatin 2 & 6.8 & 559.5 & 250.2 & Atorvastatin D5 & & & & & \\
\hline Iopromide 1 & 4.15 & 792 & 573.1 & lopromide D3 & 0.17 & 0.50 & 0.00 & n.d. & 11 \\
\hline Iopromide 2 & 4.15 & 792 & 559.1 & Iopromide D3 & & & & & \\
\hline Haloxyfop-Me 1 & 7.04 & 376 & 316 & & & & & & \\
\hline Haloxyfop-Me 2 & 7.04 & 376 & 288 & & & & & & \\
\hline Haloxyfop-Me 4 & 7.04 & 376 & 272 & & & & & & \\
\hline
\end{tabular}


Table S-6. MS and quality assurance information for PPCPs and pesticides analysed in ESI negative mode.

\begin{tabular}{|c|c|c|c|c|c|c|c|c|c|}
\hline Analyte ID negative & $\begin{array}{r}\begin{array}{r}\text { Retention time } \\
\text { (Min) }\end{array} \\
\end{array}$ & $\begin{array}{r}\text { Q1 Mass } \\
(\mathrm{Da}) \\
\end{array}$ & $\begin{array}{r}\text { Q3 Mass } \\
(\mathrm{Da}) \\
\end{array}$ & Mass labelled & $\begin{array}{r}\text { ILOD } \\
\text { (ng/mL) } \\
\end{array}$ & $\begin{array}{r}\text { ILOQ } \\
(\mathrm{ng} / \mathrm{mL})\end{array}$ & $\begin{array}{r}\text { LOR } \\
(\mathrm{ng} / \mathrm{mL}) \\
\end{array}$ & $\begin{array}{r}\text { MilliQ blank } \\
(\mathrm{ng} / \mathrm{mL})\end{array}$ & $\begin{array}{r}\text { Repeatability } \\
(\% \mathrm{CV})\end{array}$ \\
\hline Salicylic acid 1 & 4.43 & 137 & 93 & & 0.16 & 0.49 & 59.48 & 26.44 & 7 \\
\hline Salicylic acid 2 & 4.43 & 137 & 65 & & & & & & \\
\hline Acesulfame 1 & 1.13 & 162 & 82 & Acesulfame D4 & 0.13 & 0.40 & 214.20 & 95.20 & 6 \\
\hline Acesulfame 2 & 1.13 & 162 & 78 & Acesulfame D4 & & & & & \\
\hline MCPA 1 & 6 & 199 & 141 & MCPA D6 & 0.18 & 0.53 & 0.00 & 0.00 & 6 \\
\hline MCPA 2 & 6 & 201 & 143 & MCPA D6 & & & & & \\
\hline Ibuprofen 1 & 6.56 & 205.1 & 161 & Ibuprofen D3 & 0.15 & 0.45 & 0.12 & 0.05 & 9 \\
\hline Ibuprofen 2 & 6.56 & 205.1 & 159 & Ibuprofen D3 & & & & & \\
\hline Mecoprop 1 & 6.21 & 213 & 141 & & 0.11 & 0.34 & 0.00 & 0.00 & 5 \\
\hline Mecoprop 2 & 6.21 & 215 & 143 & & & & & & \\
\hline 2,4-D 1 & 5.94 & 219 & 161 & 2,4-D $13 C 6$ & 0.20 & 0.61 & 0.08 & 0.03 & 9 \\
\hline 2,4-D 2 & 5.94 & 221 & 163 & 2,4-D $13 \mathrm{C} 6$ & & & & & \\
\hline Dicamba 1 & 5.15 & 219 & 175 & & 0.36 & 1.08 & 5.45 & 2.42 & 18 \\
\hline Dicamba 2 & 5.15 & 221 & 177 & & & & & & \\
\hline 2,4-DB 1 & 6.51 & 247 & 161 & & & & 0.00 & 0.00 & \\
\hline 2,4-DB 2 & 6.51 & 249 & 163 & & & & & & \\
\hline $2,4,5-\mathrm{T} 1$ & 6.3 & 252.9 & 194.9 & & 0.31 & 0.93 & 0.00 & 0.00 & 13 \\
\hline 2,4,5-Т 2 & 6.3 & 254.9 & 196.9 & & & & & & \\
\hline Triclopyr 1 & 6.31 & 254 & 196 & & 0.37 & 1.10 & 0.00 & 0.00 & 23 \\
\hline Triclopyr 2 & 6.31 & 256 & 198 & & & & & & \\
\hline Triclopyr 3 & 6.31 & 255.9 & 200 & & & & & & \\
\hline Bromoxynil 1 & 5.9 & 273.8 & 78.9 & & 0.21 & 0.63 & 0.01 & 0.01 & 11 \\
\hline Bromoxynil 2 & 5.9 & 275.8 & 78.9 & & & & & & \\
\hline Triclosan 1 & 6.95 & 287 & 35 & & 0.10 & 0.29 & 0.92 & 0.41 & 7 \\
\hline $\begin{array}{r}\text { Triclosan } 2 \\
\text { Hydrochlorothiazide }\end{array}$ & 6.95 & 289 & 35 & & & & & & \\
\hline 1 & 2.96 & 296 & 269 & Hydrochlorothiazide $13 \mathrm{CD} 2$ & 0.07 & 0.22 & 0.87 & 0.39 & 4 \\
\hline Hydrochlorothiazide & & & & & & & & & \\
\hline 2 & 2.96 & 296 & 205 & Hydrochlorothiazide $13 C D 2$ & & & & & \\
\hline Furosemide 1 & 5.78 & 329 & 285 & & 0.40 & 1.21 & 3.34 & 1.48 & 19 \\
\hline Furosemide 2 & 5.78 & 329 & 205 & & & & & & \\
\hline Diketonitrile- 1 & 5.65 & 358.2 & 79 & & 0.08 & 0.25 & 0.02 & 0.01 & 4 \\
\hline Diketonitrile- 2 & 5.65 & 358.2 & 64 & & & & & & \\
\hline Haloxyfop- 1 & 6.72 & 360 & 288 & Haloxyfop D4- & 0.22 & 0.67 & 0.05 & 0.02 & 9 \\
\hline Haloxyfop- 2 & 6.72 & 362 & 290 & Haloxyfop D4- & & & & & \\
\hline
\end{tabular}


Table S-7. MS information for labelled PPCP and pesticide standards.

POSITIVE

\begin{tabular}{|c|c|c|c|}
\hline Analyte ID & $\begin{array}{c}\text { Retention time } \\
\text { (Min) }\end{array}$ & $\begin{array}{c}\text { Q1 Mass } \\
\text { (Da) }\end{array}$ & $\begin{array}{l}\text { Q3 Mass } \\
\text { (Da) }\end{array}$ \\
\hline Paracetamol D4 1 & 1.54 & 156.1 & 114.1 \\
\hline Paracetamol D4 2 & 1.54 & 156.1 & 69.1 \\
\hline Methomyl D3 1 & 4.38 & 166 & 88 \\
\hline Methomyl D3 2 & 4.38 & 166 & 106 \\
\hline Nicotine D4 1 & 0.87 & 167.1 & 136 \\
\hline Nicotine D4 2 & 0.87 & 167.1 & 121 \\
\hline Atrazine Desisopropyl D5 1 & 4.04 & 179.1 & 137.1 \\
\hline Atrazine Desisopropyl D5 2 & 4.04 & 179.1 & 101.2 \\
\hline Cotinine D3 1 & 3.35 & 180.1 & 80 \\
\hline Cotinine D3 2 & 3.35 & 180.1 & 101 \\
\hline Gabapentin D10 1 & 3.01 & 182.1 & 164 \\
\hline Gabapentin D10 2 & 3.01 & 182.1 & 147 \\
\hline Paraxanthine D3 1 & 3.98 & 184 & 127 \\
\hline Paraxanthine D3 3 & 3.98 & 184 & 72 \\
\hline 5HIAA D2 1 & 0.1 & 194.1 & 148 \\
\hline 5HIAA D2 2 & 0.1 & 194.1 & 120 \\
\hline Atrazine desethyl D6 1 & 4.78 & 194.2 & 147.1 \\
\hline Atrazine desethyl D6 2 & 4.78 & 194.2 & 111.1 \\
\hline Hydroxycotinine D3 1 & 1.37 & 196.1 & 134.1 \\
\hline Hydroxycotinine D3 2 & 1.37 & 196.1 & 80 \\
\hline Caffeine $13 C 31$ & 4.75 & 198.3 & 140.1 \\
\hline Caffeine $13 C 32$ & 4.75 & 198.3 & 112.1 \\
\hline DEET D7 1 & 6.24 & 199.2 & 126.1 \\
\hline DEET D7 2 & 6.24 & 199.2 & 98.2 \\
\hline Tebuthylazine desethyl D9 1 & 5.52 & 211.1 & 147 \\
\hline Tebuthylazine desethyl D9 2 & 5.52 & 211.1 & 105 \\
\hline Simazine D10 1 & 5.52 & 212 & 137 \\
\hline Simazine D10 2 & 5.52 & 212 & 134 \\
\hline Propoxur D3 1 & 5.73 & 213.1 & 171.1 \\
\hline Propoxur D3 2 & 5.73 & 213.1 & 111 \\
\hline Metribuzin D3 1 & 5.74 & 218 & 190 \\
\hline Metribuzin D3 2 & 5.74 & 218 & 84 \\
\hline Atrazine D5 1 & 5.87 & 221.1 & 179 \\
\hline Atrazine D5 2 & 5.87 & 221.1 & 101 \\
\hline Carbofuran D3 1 & 5.88 & 225 & 165 \\
\hline Carbofuran D3 2 & 5.88 & 225 & 123 \\
\hline Methiocarb D3 1 & 6.4 & 229.1 & 169 \\
\hline Methiocarb D3 2 & 6.4 & 229.1 & 121 \\
\hline Terbuthylazine D5 1 & 6.23 & 235.1 & 179 \\
\hline Terbuthylazine D5 2 & 6.23 & 235.1 & 101 \\
\hline Propazine D6 1 & 6.14 & 236 & 147 \\
\hline Propazine D6 2 & 6.14 & 236 & 194 \\
\hline Diuron D6 1 & 5.86 & 240.9 & 78.2 \\
\hline
\end{tabular}


POSITIVE

\begin{tabular}{|c|c|c|c|}
\hline Analyte ID & $\begin{array}{c}\text { Retention time } \\
\text { (Min) }\end{array}$ & $\begin{array}{l}\text { Q1 Mass } \\
\text { (Da) }\end{array}$ & $\begin{array}{l}\text { Q3 Mass } \\
\text { (Da) }\end{array}$ \\
\hline Diuron D6 2 & 5.86 & 240.9 & 52.1 \\
\hline Carbamazepine D10 1 & 6.08 & 247.2 & 204.1 \\
\hline Carbamazepine D10 2 & 6.08 & 247.2 & 202.1 \\
\hline Prometryn D6 1 & 6.3 & 248.2 & 159 \\
\hline Prometryn D6 2 & 6.3 & 248.2 & 206 \\
\hline Hexazinone D6 1 & 6.08 & 259.3 & 177.2 \\
\hline Hexazinone D6 2 & 6.08 & 259.3 & 77.2 \\
\hline Imidacloprid D4 1 & 5.25 & 260.2 & 179.3 \\
\hline Imidacloprid D4 2 & 5.25 & 260.2 & 213.2 \\
\hline Bromacil D3 1 & 5.55 & 264.1 & 208 \\
\hline Bromacil D3 2 & 5.55 & 266.1 & 210 \\
\hline Tramadol D6 1 & 4.69 & 268.2 & 58 \\
\hline Tramadol D6 2 & 4.69 & 268.2 & 42 \\
\hline Atenolol D7 1 & 2.76 & 274.1 & 145.1 \\
\hline Atenolol D7 2 & 2.76 & 274.1 & 190.1 \\
\hline Venlafaxine D6 1 & 5.05 & 284.2 & 64 \\
\hline Venlafaxine D6 2 & 5.05 & 284.2 & 121 \\
\hline Metolachlor D6 1 & 6.86 & 290.2 & 258.2 \\
\hline Metolachlor D6 2 & 6.86 & 290.2 & 182.2 \\
\hline D4 Acetyl Sulfamethoxazole 1 & 0.1 & 300.1 & 138.1 \\
\hline D4 Acetyl Sulfamethoxazole 2 & 0.1 & 300.1 & 69.1 \\
\hline Codeine D3 1 & 4.01 & 303.3 & 152 \\
\hline Codeine D3 2 & 4.01 & 303.3 & 115 \\
\hline Temazepam D5 1 & 6.69 & 306.2 & 260.1 \\
\hline Temazepam D5 2 & 6.69 & 306.2 & 288.1 \\
\hline Tebuconazole D6 1 & 6.68 & 314.2 & 72 \\
\hline Tebuconazole D6 2 & 6.68 & 314.2 & 125 \\
\hline Diazinon D10 1 & 6.84 & 315.1 & 170 \\
\hline Diazinon D10 2 & 6.84 & 315.1 & 154 \\
\hline Fluoxetine D6 1 & 5.54 & 316.2 & 44 \\
\hline Fluoxetine D6 2 & 5.54 & 316.2 & 154.2 \\
\hline Norfloxacin D5 1 & 4.31 & 325.1 & 231.1 \\
\hline Norfloxacin D5 2 & 4.31 & 325.1 & 281.2 \\
\hline Citalopram D6 1 & 5.34 & 331.1 & 109 \\
\hline Citalopram D6 2 & 5.34 & 331.1 & 262.1 \\
\hline Fluazifop D4+ 1 & 6.38 & 332.1 & 285.2 \\
\hline Fluazifop D4+ 2 & 6.38 & 332.1 & 257.1 \\
\hline Propiconazole D5 1 & 6.97 & 347 & 159 \\
\hline Propiconazole D5 2 & 6.97 & 349 & 161 \\
\hline Propiconazole D5 3 & 6.97 & 347 & 123 \\
\hline Haloxyfop D4+ 1 & 6.72 & 366 & 319.2 \\
\hline Haloxyfop D4+ 2 & 6.72 & 366 & 291.2 \\
\hline Metsulfuron-methyl D3 1 & 6.3 & 385.1 & 170 \\
\hline Metsulfuron-methyl D3 2 & 6.3 & 385.1 & 144 \\
\hline Atorvastatin D5 1 & 6.8 & 564.3 & 445.3 \\
\hline Atorvastatin D5 2 & 6.8 & 564.3 & 255.1 \\
\hline
\end{tabular}


POSITIVE

\begin{tabular}{|c|c|c|c|}
\hline Analyte ID & $\begin{array}{c}\text { Retention time } \\
\text { (Min) }\end{array}$ & $\begin{array}{c}\text { Q1 Mass } \\
\text { (Da) }\end{array}$ & $\begin{array}{c}\text { Q3 Mass } \\
\text { (Da) }\end{array}$ \\
\hline Iopromide D3 1 & 4.15 & 794.8 & 575.9 \\
\hline lopromide D3 2 & 4.15 & 794.8 & 561.9 \\
\hline \multicolumn{4}{|c|}{ NEGATIVE } \\
\hline \multicolumn{4}{|c|}{ Retention time } \\
\hline Analyte ID & (Min) & Q1 Mass (Da) & Q3 Mass (Da) \\
\hline Acesulfame D4 1 & 1.11 & 166 & 86 \\
\hline Acesulfame D4 2 & 1.11 & 166 & 78 \\
\hline DCPA int std 1 & 0.1 & 205 & 161 \\
\hline DCPA int std 2 & 0.1 & 203 & 159 \\
\hline MCPA D6 1 & 5.98 & 205.1 & 147.1 \\
\hline MCPA D6 2 & 5.98 & 207.1 & 149.1 \\
\hline 2,4-D $13 C 61$ & 5.94 & 225 & 167 \\
\hline 2,4-D $13 C 62$ & 5.94 & 227 & 169 \\
\hline Hydrochlorothiazide 13CD2 1 & 2.94 & 298.9 & 269.9 \\
\hline Hydrochlorothiazide 13CD2 2 & 2.94 & 298.9 & 205.9 \\
\hline Haloxyfop D4- 1 & 6.71 & 364 & 292 \\
\hline Haloxyfop D4- 2 & 6.71 & 366 & 294 \\
\hline Ibuprofen D3 1 & 6.55 & 208.1 & 164 \\
\hline Ibuprofen D3 2 & 6.55 & 208.1 & 161 \\
\hline
\end{tabular}


Table S-8. Daily PFASs discharge from Goodna WWTP in the upper stretch of the Brisbane River estuary between Aug. 6 (Day 1) and Aug. 12 (Day 7) 2017.

\begin{tabular}{|c|c|c|c|c|c|c|c|c|}
\hline & \multicolumn{8}{|c|}{ Goodna WWTP effluent discharge (mg/day) } \\
\hline & Day 1 & Day 2 & Day 3 & Day 4 & Day 5 & Day 6 & Day 7 & $\mathrm{CV} \%^{\S}$ \\
\hline PFBA & 29.83 & 35.28 & 28.68 & 28.33 & 27.19 & 22.63 & 25.16 & 14 \\
\hline PFPeA & 141.99 & 132.00 & 129.39 & 116.93 & 129.62 & 113.34 & 114.64 & 9 \\
\hline PFHXA & 248.44 & 238.81 & 223.21 & 216.17 & 219.74 & 209.31 & 212.51 & 6 \\
\hline PFHpA & n.d. & n.d. & n.d. & n.d. & n.d. & n.d. & n.d. & - \\
\hline PFOA & 353.56 & 349.67 & 314.84 & 304.97 & 324.33 & 316.77 & 332.38 & 6 \\
\hline PFNA & n.d. & n.d. & n.d. & n.d. & n.d. & n.d. & n.d. & - \\
\hline PFDA & 30.19 & 35.40 & n.d. & 27.47 & 27.81 & 27.63 & 31.70 & 10 \\
\hline PFUnDA & n.d. & n.d. & n.d. & n.d. & n.d. & n.d. & n.d. & - \\
\hline PFDoDA & n.d. & n.d. & n.d. & n.d. & n.d. & n.d. & n.d. & - \\
\hline PFTrDA & n.d. & n.d. & n.d. & n.d. & n.d. & n.d. & n.d. & - \\
\hline PFTeDA & n.d. & n.d. & n.d. & n.d. & n.d. & n.d. & n.d. & - \\
\hline PFHxDA & $<\mathrm{LOQ}$ & $<L O Q$ & $<L O Q$ & $<L O Q$ & $<L O Q$ & $<\mathrm{LOQ}$ & $<\mathrm{LOQ}$ & - \\
\hline PFODA & n.d. & n.d. & n.d. & n.d. & n.d. & n.d. & n.d. & - \\
\hline PFBS & n.d. & n.d. & n.d. & n.d. & n.d. & n.d. & n.d. & - \\
\hline PFPeS & $<L O Q$ & $<L O Q$ & $<L O Q$ & $<L O Q$ & $<L O Q$ & $<L O Q$ & $<\mathrm{LOQ}$ & - \\
\hline PFHxS & 21.24 & 19.72 & 17.82 & 31.45 & 20.42 & 17.96 & 20.12 & 22 \\
\hline PFHpS & $<L O Q$ & $<L O Q$ & $<L O Q$ & $<L O Q$ & $<L O Q$ & $<L O Q$ & $<\mathrm{LOQ}$ & - \\
\hline PFOS & n.d. & n.d. & n.d. & n.d. & n.d. & n.d. & n.d. & - \\
\hline PFNS & n.d. & n.d. & n.d. & n.d. & n.d. & n.d. & n.d. & - \\
\hline PFDS & n.d. & n.d. & n.d. & n.d. & n.d. & n.d. & n.d. & - \\
\hline PFDODS & n.d. & n.d. & n.d. & n.d. & n.d. & n.d. & n.d. & - \\
\hline FOSA & n.d. & n.d. & n.d. & n.d. & n.d. & n.d. & n.d. & - \\
\hline FOSAA & n.d. & n.d. & n.d. & n.d. & n.d. & n.d. & n.d. & - \\
\hline N-Et FOSAA & n.d. & n.d. & n.d. & n.d. & n.d. & n.d. & n.d. & - \\
\hline N-Me FOSAA & n.d. & n.d. & n.d. & n.d. & n.d. & n.d. & n.d. & - \\
\hline N-Me FOSA & n.d. & n.d. & n.d. & n.d. & n.d. & n.d. & n.d. & - \\
\hline N-Et FOSA & n.d. & n.d. & n.d. & n.d. & n.d. & n.d. & n.d. & - \\
\hline 10:2 FTS & n.d. & n.d. & n.d. & n.d. & n.d. & n.d. & n.d. & - \\
\hline $8: 2$ FTS & n.d. & n.d. & n.d. & n.d. & n.d. & n.d. & n.d. & - \\
\hline $6: 2$ FTS & $<\mathrm{LOQ}$ & $<L O Q$ & $<L O Q$ & $<L O Q$ & $<\mathrm{LOQ}$ & $<\mathrm{LOQ}$ & $<\mathrm{LOQ}$ & - \\
\hline 4:2 FTS & n.d. & n.d. & n.d. & n.d. & n.d. & n.d. & n.d. & - \\
\hline N-Me FOSE & n.d. & n.d. & n.d. & n.d. & n.d. & n.d. & n.d. & - \\
\hline $\mathrm{N}$-Et FOSE & n.d. & n.d. & n.d. & n.d. & n.d. & n.d. & n.d. & - \\
\hline PFECHS & n.d. & n.d. & n.d. & n.d. & n.d. & n.d. & n.d. & - \\
\hline $6: 2$ PAP & n.d. & n.d. & n.d. & n.d. & n.d. & n.d. & n.d. & - \\
\hline 8:2 PAP & n.d. & n.d. & n.d. & n.d. & n.d. & n.d. & n.d. & - \\
\hline SAmPAP & n.d. & n.d. & n.d. & n.d. & n.d. & n.d. & n.d. & - \\
\hline 6:2 DiPAP & n.d. & n.d. & n.d. & n.d. & n.d. & n.d. & n.d. & - \\
\hline 8:2 DiPAP & n.d. & n.d. & n.d. & n.d. & n.d. & n.d. & n.d. & - \\
\hline $6: 28: 2$ DiPAP & n.d. & n.d. & n.d. & n.d. & n.d. & n.d. & n.d. & - \\
\hline
\end{tabular}

${ }^{\S} \mathrm{CV}=$ coefficient of variation. 
Table S-9. Daily PPCPs and pesticides discharge from Goodna WWTP in the upper stretch of the Brisbane River estuary between Aug. 6 (Day 1) and Aug. 12 (Day 7) 2017.

\begin{tabular}{|c|c|c|c|c|c|c|c|c|}
\hline & \multicolumn{8}{|c|}{ Goodna WWTP effluent discharge (mg/day) } \\
\hline & Day 1 & Day 2 & Day 3 & Day 4 & Day 5 & Day 6 & Day 7 & CV\% \\
\hline Salicylic acid & n.d. & n.d. & n.d. & n.d. & n.d. & n.d. & n.d. & - \\
\hline Acesulfame & 4052.14 & 3048.63 & 2411.18 & 2401.22 & 3401.18 & 3459.25 & 4984.43 & 27 \\
\hline MCPA & 918.75 & 1098.16 & 509.58 & 619.93 & 508.73 & 823.31 & 796.19 & 29 \\
\hline Ibuprofen & n.d. & n.d. & n.d. & n.d. & n.d. & n.d. & n.d. & - \\
\hline Mecoprop & $<\mathrm{LOQ}$ & $<\mathrm{LOQ}$ & $<\mathrm{LOQ}$ & $<\mathrm{LOQ}$ & $<\mathrm{LOQ}$ & $<\mathrm{LOQ}$ & $<\mathrm{LOQ}$ & - \\
\hline 2,4-D & 80.13 & 85.68 & 70.98 & 77.96 & 73.78 & 89.37 & 87.38 & 9 \\
\hline Dicamba & n.d. & n.d. & n.d. & n.d. & n.d. & n.d. & n.d. & - \\
\hline $2,4-\mathrm{DB}$ & n.d. & n.d. & n.d. & n.d. & n.d. & n.d. & n.d. & - \\
\hline $2,4,5-\mathrm{T}$ & n.d. & n.d. & n.d. & n.d. & n.d. & n.d. & n.d. & - \\
\hline Triclopyr & 163.02 & 120.42 & 98.16 & 181.95 & 124.91 & 76.92 & 83.67 & 33 \\
\hline Bromoxynil & n.d. & n.d. & n.d. & n.d. & n.d. & n.d. & n.d. & - \\
\hline Triclosan & 86.95 & 215.15 & 152.29 & 138.22 & 102.71 & 60.74 & 241.74 & 47 \\
\hline Hydrochlorothiazide & 5701.91 & 5336.66 & 5176.19 & 4906.97 & 4993.58 & 4575.21 & 4839.62 & 7 \\
\hline Furosemide & n.d. & n.d. & n.d. & n.d. & n.d. & n.d. & n.d. & - \\
\hline Diketonitrile & n.d. & n.d. & n.d. & n.d. & n.d. & n.d. & n.d. & - \\
\hline Haloxyfop - & 42.73 & 46.47 & 36.15 & 44.23 & 41.82 & 50.10 & 41.30 & 10 \\
\hline Paracetamol & 2546.62 & 381.83 & 10371.57 & 186.84 & 1120.17 & 937.78 & 226.25 & 163 \\
\hline 3,4 DiCl Aniline & 16.96 & 98.31 & 85.08 & 66.72 & 32.96 & 26.19 & 54.67 & 57 \\
\hline Methomyl & n.d. & n.d. & n.d. & n.d. & n.d. & n.d. & n.d. & - \\
\hline Nicotine & n.d. & n.d. & n.d. & n.d. & n.d. & n.d. & n.d. & - \\
\hline Gabapentin & $1.25 \cdot 10^{6}$ & $7.31 \cdot 10^{5}$ & $1.28 \cdot 10^{6}$ & $1.20 \cdot 10^{6}$ & $1.15 \cdot 10^{6}$ & $4.64 \cdot 10^{4}$ & $7.29 \cdot 10^{5}$ & 49 \\
\hline Desisopropyl Atrazine & n.d. & n.d. & n.d. & n.d. & n.d. & n.d. & n.d. & - \\
\hline Cotinine & 77.45 & 82.01 & 78.29 & 73.62 & 79.81 & 70.73 & 84.46 & 6 \\
\hline Paraxanthine & 455.19 & 374.14 & 294.56 & 296.42 & 294.69 & 285.62 & 401.11 & 20 \\
\hline Simazine hydroxy & n.d. & n.d. & n.d. & n.d. & n.d. & n.d. & n.d. & - \\
\hline Desethyl Atrazine & n.d. & n.d. & n.d. & n.d. & n.d. & n.d. & n.d. & - \\
\hline Clopyralid & n.d. & n.d. & n.d. & n.d. & n.d. & n.d. & n.d. & - \\
\hline 5HIAA & n.d. & n.d. & n.d. & n.d. & n.d. & n.d. & n.d. & - \\
\hline DEET & 579.20 & 418.09 & 435.00 & 333.42 & 318.23 & 311.49 & 718.61 & 34 \\
\hline Hydroxycotinine & 425.15 & 526.31 & 427.06 & 431.36 & 406.95 & 407.00 & 567.34 & 14 \\
\hline Caffeine & 233.76 & 259.07 & 198.22 & 203.65 & 285.77 & 219.65 & 234.68 & 13 \\
\hline Ametryn hydroxy & n.d. & n.d. & n.d. & n.d. & n.d. & n.d. & n.d. & - \\
\hline Pyrimethanil & 8.06 & 9.50 & 6.84 & 13.36 & 8.14 & n.d. & 7.42 & 27 \\
\hline Terbuthylazine desethyl & n.d. & n.d. & n.d. & n.d. & n.d. & n.d. & n.d. & - \\
\hline Simazine & n.d. & n.d. & n.d. & n.d. & $<\mathrm{LOQ}$ & n.d. & n.d. & - \\
\hline DCPU & n.d. & n.d. & n.d. & n.d. & n.d. & n.d. & n.d. & - \\
\hline Propoxur & 145.24 & 107.83 & 95.32 & 68.51 & 51.85 & 41.43 & 42.82 & 49 \\
\hline Metribuzin & n.d. & n.d. & n.d. & n.d. & n.d. & n.d. & n.d. & - \\
\hline Atrazine & n.d. & n.d. & n.d. & n.d. & n.d. & n.d. & n.d. & - \\
\hline DCPMU & n.d. & n.d. & n.d. & n.d. & n.d. & n.d. & n.d. & - \\
\hline Dichlorvos & n.d. & n.d. & n.d. & n.d. & n.d. & 8.26 & n.d. & - \\
\hline Carbofuran & n.d. & n.d. & n.d. & n.d. & n.d. & n.d. & n.d. & - \\
\hline Methiocarb & n.d. & n.d. & n.d. & n.d. & n.d. & n.d. & n.d. & - \\
\hline Ametryn & n.d. & n.d. & n.d. & n.d. & n.d. & n.d. & n.d. & - \\
\hline Tebuthiuron & $<\mathrm{LOQ}$ & $<\mathrm{LOQ}$ & $<\mathrm{LOQ}$ & $<L O Q$ & $<L O Q$ & $<\mathrm{LOQ}$ & $<\mathrm{LOQ}$ & - \\
\hline
\end{tabular}


Goodna WWTP effluent discharge ( $\mathrm{mg} /$ day)

\begin{tabular}{|c|c|c|c|c|c|c|c|c|}
\hline & Day 1 & Day 2 & Day 3 & Day 4 & Day 5 & Day 6 & Day 7 & CV\% \\
\hline Propazine & n.d. & n.d. & n.d. & n.d. & n.d. & n.d. & n.d. & - \\
\hline Terbuthylazine & n.d. & n.d. & n.d. & n.d. & n.d. & n.d. & n.d. & - \\
\hline Asulam & n.d. & n.d. & n.d. & n.d. & n.d. & n.d. & n.d. & - \\
\hline Naproxen & 92.56 & 113.32 & 98.49 & 106.11 & 77.75 & 97.93 & 96.15 & 11 \\
\hline Diuron & 552.81 & 524.47 & 750.52 & 604.29 & 621.74 & 546.69 & 734.52 & 15 \\
\hline Fluometuron & n.d. & n.d. & n.d. & n.d. & n.d. & n.d. & n.d. & - \\
\hline Carbamazepine & 4596.63 & 4338.89 & 4554.81 & 3913.41 & 3032.87 & 3883.69 & 4535.88 & 14 \\
\hline Prometryn & n.d. & n.d. & n.d. & n.d. & n.d. & n.d. & n.d. & - \\
\hline Terbutryn & 6.15 & 10.90 & 7.29 & 6.16 & 3.55 & 2.93 & 13.04 & 52 \\
\hline Picloram & 203.09 & 248.54 & 193.77 & 258.99 & 175.96 & 124.17 & 133.63 & 27 \\
\hline Hexazinone & 2748.58 & 1174.71 & 471.93 & 296.88 & 171.99 & 106.72 & 1414.82 & 105 \\
\hline Fluroxypyr & 62.93 & 86.94 & 79.55 & 102.99 & 92.55 & 105.52 & 73.70 & 18 \\
\hline Imidacloprid & 1673.71 & 1292.14 & 982.37 & 838.30 & 759.74 & 938.65 & 1179.54 & 29 \\
\hline Bromacil & n.d. & n.d. & n.d. & n.d. & n.d. & n.d. & n.d. & - \\
\hline Tramadol & n.d. & n.d. & n.d. & n.d. & n.d. & n.d. & n.d. & - \\
\hline Atenolol & 356.21 & 334.39 & 365.85 & 299.78 & 294.38 & 279.58 & 361.23 & 11 \\
\hline DesmethylDiazepam & 73.73 & 105.85 & 95.25 & 89.47 & 78.47 & 73.38 & 93.86 & 14 \\
\hline Imazapic & 155.75 & 477.70 & 410.95 & 272.97 & 231.46 & 111.32 & 585.88 & 55 \\
\hline Venlafaxiine & 6019.19 & 5975.26 & 5640.89 & 5800.64 & 14222.64 & 20613.01 & 5901.24 & 65 \\
\hline Metalaxyl & n.d. & n.d. & n.d. & n.d. & n.d. & n.d. & n.d. & - \\
\hline Pendimethalin & n.d. & n.d. & n.d. & n.d. & n.d. & n.d. & n.d. & - \\
\hline Metolachlor & $<\mathrm{LOQ}$ & 46.98 & 42.28 & $<\mathrm{LOQ}$ & $<\mathrm{LOQ}$ & $<\mathrm{LOQ}$ & $<\mathrm{LOQ}$ & 7 \\
\hline Imazethapyr & n.d. & n.d. & n.d. & n.d. & n.d. & n.d. & n.d. & - \\
\hline Codeine & 1262.53 & 1348.41 & 1402.97 & 1466.35 & 1501.99 & 1354.81 & 1596.40 & 8 \\
\hline Temazepam & 1021.16 & 659.25 & 641.31 & 780.16 & 912.62 & 795.40 & 947.98 & 18 \\
\hline Fenamiphos & n.d. & n.d. & n.d. & n.d. & n.d. & n.d. & n.d. & - \\
\hline Diazinon & n.d. & n.d. & n.d. & n.d. & n.d. & n.d. & n.d. & - \\
\hline Tebuconazole & 148.14 & 118.75 & 127.83 & 166.99 & 177.53 & 218.41 & 244.77 & 27 \\
\hline Fluoxetine & 277.04 & 217.45 & 232.82 & 214.76 & 241.72 & 245.84 & 236.30 & 9 \\
\hline Desmethyl Citalopram & 921.16 & 1952.79 & 2010.02 & 1346.77 & 1158.65 & 725.59 & 1970.48 & 37 \\
\hline Citalopram & 2384.29 & 2289.29 & 1508.76 & 2331.65 & 2581.95 & 1918.35 & 1632.41 & 20 \\
\hline Fluazifop & $<\mathrm{LOQ}$ & 73.80 & 65.22 & $<\mathrm{LOQ}$ & $<\mathrm{LOQ}$ & n.d. & 147.94 & 48 \\
\hline Malathion & n.d. & n.d. & n.d. & n.d. & n.d. & n.d. & n.d. & - \\
\hline Propiconazole & 192.02 & 149.34 & 130.19 & 194.33 & 207.09 & 254.40 & 183.23 & 21 \\
\hline Prothioconazole & n.d. & n.d. & n.d. & n.d. & n.d. & n.d. & n.d. & - \\
\hline Chlorpyriphos & n.d. & n.d. & n.d. & n.d. & n.d. & n.d. & n.d. & - \\
\hline Haloxyfop + & n.d. & n.d. & n.d. & n.d. & n.d. & n.d. & n.d. & - \\
\hline Metsulfuron-Methyl & n.d. & n.d. & n.d. & n.d. & n.d. & n.d. & n.d. & - \\
\hline Tadalafil & n.d. & n.d. & 7.75 & 6.05 & n.d. & n.d. & n.d. & 17 \\
\hline Verapamil & 199.60 & 331.01 & 305.96 & 313.61 & 230.88 & 125.42 & 590.22 & 49 \\
\hline Sildenafil & 142.21 & 408.35 & 316.85 & 230.69 & 152.19 & 77.65 & 364.28 & 52 \\
\hline Atorvastatin & n.d. & n.d. & n.d. & n.d. & n.d. & n.d. & n.d. & - \\
\hline Iopromide & $1.57 \cdot 10^{4}$ & $7.31 \cdot 10^{4}$ & $8.21 \cdot 10^{4}$ & $5.84 \cdot 10^{4}$ & $4.12 \cdot 10^{4}$ & $4.84 \cdot 10^{4}$ & $3.78 \cdot 10^{4}$ & 44 \\
\hline
\end{tabular}

${ }^{\S} \mathrm{CV}=$ coefficient of variation. 
Table S-10. PFAS concentrations in surface water samples.

\begin{tabular}{|c|c|c|c|c|c|c|c|c|c|c|c|c|c|c|c|c|}
\hline $\begin{array}{l}\text { Concentration } \\
\text { (ng/L) }\end{array}$ & PFBA & PFPeA & PFHxA & PFHpA & PFOA & PFNA & PFDA & PFUnDA & PFDoDA & PFTrDA & PFTeDA & PFHxDA & PFODA & PFBS & PFPeS & PFHxS \\
\hline 29/03/2017 & 10.89 & 11.69 & 17.07 & n.d. & 11.91 & 1.77 & 2.76 & 0.39 & $<\mathrm{LOQ}$ & n.d. & n.d. & n.d. & n.d. & 6.38 & 3.48 & 23.55 \\
\hline $31 / 03 / 2017$ & 54.06 & 4.25 & 5.27 & n.d. & 3.64 & n.d. & 0.61 & $<\mathrm{LOQ}$ & $<\mathrm{LOQ}$ & n.d. & n.d. & n.d. & n.d. & 1.83 & 1.05 & 9.01 \\
\hline 01/04/2017 & 8.28 & 7.00 & 8.16 & n.d. & 5.15 & 0.96 & 0.95 & $<\mathrm{LOQ}$ & $<\mathrm{LOQ}$ & n.d. & n.d. & n.d. & n.d. & 2.48 & 1.52 & 10.88 \\
\hline $02 / 04 / 2017$ & 3.58 & n.d. & 1.33 & n.d. & 0.75 & n.d. & $<\mathrm{LOQ}$ & n.d. & n.d. & n.d. & n.d. & n.d. & n.d. & 0.55 & n.d. & 2.53 \\
\hline 03/04/2017 & 4.16 & 3.10 & 3.70 & n.d. & 2.45 & n.d. & 0.41 & n.d. & n.d. & n.d. & n.d. & n.d. & n.d. & 1.17 & 0.68 & 5.47 \\
\hline 04/04/2017 & 4.45 & 1.76 & 2.63 & n.d. & 1.81 & n.d. & 0.32 & n.d. & n.d. & n.d. & n.d. & n.d. & n.d. & 0.97 & 0.47 & 4.26 \\
\hline 05/04/2017 & 1.71 & 1.57 & 2.38 & n.d. & 1.49 & n.d. & $<\mathrm{LOQ}$ & n.d. & n.d. & n.d. & n.d. & n.d. & n.d. & 0.87 & 0.42 & 4.01 \\
\hline 06/04/2017 & 4.16 & 1.57 & 2.28 & n.d. & 1.31 & n.d. & $<\mathrm{LOQ}$ & n.d. & n.d. & n.d. & n.d. & n.d. & n.d. & 0.81 & 0.41 & 3.78 \\
\hline 07/04/2017 & 3.55 & 1.46 & 2.28 & n.d. & 1.30 & n.d. & $<\mathrm{LOQ}$ & n.d. & n.d. & n.d. & n.d. & n.d. & $<\mathrm{LOQ}$ & 0.99 & 0.42 & 3.80 \\
\hline 09/04/2017 & 2.47 & 1.78 & 2.56 & n.d. & 1.52 & n.d. & 0.26 & n.d. & n.d. & n.d. & n.d. & n.d. & n.d. & 1.03 & 0.46 & 4.26 \\
\hline $10 / 04 / 2017$ & 2.41 & 1.60 & 2.69 & n.d. & 1.57 & n.d. & $<\mathrm{LOQ}$ & n.d. & n.d. & n.d. & n.d. & n.d. & n.d. & 1.14 & 0.52 & 4.28 \\
\hline $12 / 04 / 2017$ & 3.65 & 1.86 & 2.74 & n.d. & 1.60 & n.d. & $<\mathrm{LOQ}$ & n.d. & n.d. & n.d. & n.d. & n.d. & n.d. & 1.22 & 0.50 & 4.06 \\
\hline $14 / 04 / 2017$ & 4.78 & 1.90 & 3.05 & n.d. & 1.87 & n.d. & 0.34 & n.d. & n.d. & n.d. & n.d. & n.d. & n.d. & 1.28 & 0.52 & 4.38 \\
\hline $15 / 04 / 2017$ & 4.64 & 2.08 & 3.38 & n.d. & 2.17 & n.d. & 0.29 & n.d. & n.d. & n.d. & n.d. & n.d. & n.d. & 1.56 & 0.61 & 5.10 \\
\hline $17 / 04 / 2017$ & 3.03 & 2.27 & 3.34 & n.d. & 2.13 & n.d. & 0.37 & n.d. & n.d. & n.d. & n.d. & n.d. & n.d. & 1.59 & 0.58 & 4.66 \\
\hline $19 / 04 / 2017$ & 3.94 & 2.24 & 3.63 & n.d. & 2.48 & n.d. & 0.32 & n.d. & n.d. & n.d. & n.d. & n.d. & n.d. & 1.33 & 0.61 & 4.62 \\
\hline 26/04/2017 & 4.65 & 2.63 & 4.09 & n.d. & 2.68 & n.d. & 0.36 & n.d. & n.d. & n.d. & n.d. & n.d. & n.d. & 1.54 & 0.70 & 5.34 \\
\hline 03/05/2017 & 3.18 & 2.73 & 4.27 & n.d. & 3.07 & n.d. & 0.65 & n.d. & n.d. & n.d. & n.d. & n.d. & $<\mathrm{LOQ}$ & 1.75 & 0.66 & 5.33 \\
\hline $11 / 05 / 2017$ & 5.04 & 2.64 & 4.02 & n.d. & 2.89 & n.d. & 0.42 & n.d. & n.d. & n.d. & n.d. & n.d. & n.d. & 1.43 & 0.62 & 5.05 \\
\hline $17 / 05 / 2017$ & 4.37 & 2.99 & 4.43 & n.d. & 3.26 & n.d. & 0.47 & n.d. & n.d. & n.d. & n.d. & n.d. & n.d. & 1.63 & 0.67 & 5.04 \\
\hline $24 / 05 / 2017$ & 5.59 & 3.35 & 5.40 & n.d. & 4.64 & 1.04 & 0.55 & n.d. & n.d. & n.d. & n.d. & n.d. & n.d. & 1.85 & 0.81 & 5.66 \\
\hline $31 / 05 / 2017$ & 5.99 & 3.94 & 5.69 & n.d. & 4.68 & n.d. & 0.68 & n.d. & n.d. & n.d. & n.d. & n.d. & n.d. & 2.09 & 0.88 & 6.53 \\
\hline 07/06/2017 & 4.39 & 3.62 & 5.76 & n.d. & 4.76 & n.d. & 0.65 & n.d. & $<\mathrm{LOQ}$ & n.d. & n.d. & n.d. & n.d. & 1.77 & 0.80 & 5.77 \\
\hline $21 / 06 / 2017$ & 7.10 & 4.48 & 6.96 & n.d. & 10.27 & 0.81 & 0.82 & n.d. & n.d. & n.d. & n.d. & n.d. & n.d. & 2.07 & 0.93 & 9.25 \\
\hline 29/06/2017 & 6.77 & 4.33 & 6.86 & n.d. & 6.54 & 0.72 & 0.98 & n.d. & $<\mathrm{LOQ}$ & n.d. & n.d. & n.d. & n.d. & 2.17 & 0.87 & 6.85 \\
\hline 05/07/2017 & 5.07 & 4.57 & 7.45 & n.d. & 6.93 & 0.92 & 1.18 & n.d. & $<\mathrm{LOQ}$ & n.d. & n.d. & n.d. & n.d. & 2.21 & 1.02 & 6.96 \\
\hline $19 / 07 / 2017$ & 6.25 & 5.06 & 8.43 & n.d. & 7.61 & 0.89 & 0.93 & n.d. & n.d. & $<\mathrm{LOQ}$ & n.d. & n.d. & n.d. & 2.54 & 1.16 & 8.14 \\
\hline $27 / 07 / 2017$ & 7.54 & 5.12 & 8.48 & n.d. & 7.99 & 0.82 & 1.01 & n.d. & $<\mathrm{LOQ}$ & $<\mathrm{LOQ}$ & n.d. & n.d. & n.d. & 2.64 & 1.26 & 8.61 \\
\hline 02/08/2017 & 6.82 & 4.82 & 9.19 & n.d. & 7.40 & 0.83 & 1.09 & n.d. & $<\mathrm{LOQ}$ & n.d. & n.d. & n.d. & n.d. & 2.93 & 1.41 & 9.16 \\
\hline $30 / 08 / 2017$ & 9.41 & 6.28 & 11.09 & n.d. & 11.04 & 1.18 & 1.74 & $<\mathrm{LOQ}$ & $<\mathrm{LOQ}$ & $<\mathrm{LOQ}$ & n.d. & n.d. & n.d. & 3.91 & 1.49 & 9.70 \\
\hline $17 / 09 / 2017$ & 7.54 & 6.02 & 10.52 & n.d. & 10.56 & 0.97 & 1.13 & n.d. & $<\mathrm{LOQ}$ & n.d. & n.d. & n.d. & n.d. & 3.02 & 1.28 & 8.42 \\
\hline 27/09/2017 & 11.80 & 6.10 & 12.28 & n.d. & 13.18 & 1.09 & 1.21 & n.d. & $<\mathrm{LOQ}$ & n.d. & n.d. & n.d. & n.d. & 3.14 & 1.56 & 9.53 \\
\hline $04 / 10 / 2017$ & 3.46 & 5.01 & 10.42 & n.d. & 11.05 & 0.93 & 1.20 & n.d. & $<\mathrm{LOQ}$ & n.d. & n.d. & n.d. & n.d. & 2.79 & 1.31 & 8.59 \\
\hline $18 / 10 / 2017$ & 7.97 & 7.97 & 13.52 & n.d. & 12.21 & 1.29 & 1.77 & $<\mathrm{LOQ}$ & $<\mathrm{LOQ}$ & n.d. & $<\mathrm{LOQ}$ & n.d. & n.d. & 3.69 & 1.87 & 11.48 \\
\hline 01/11/2017 & 9.43 & 7.88 & 13.69 & n.d. & 13.01 & 1.41 & 1.43 & n.d. & $<\mathrm{LOQ}$ & n.d. & n.d. & n.d. & n.d. & 4.68 & 2.09 & 13.72 \\
\hline $16 / 11 / 2017$ & 7.57 & 6.47 & 11.71 & 9.64 & 12.12 & 1.80 & 1.72 & n.d. & $<\mathrm{LOQ}$ & n.d. & n.d. & n.d. & n.d. & 4.53 & 1.81 & 11.56 \\
\hline $12 / 12 / 2017$ & 7.23 & 7.04 & 13.27 & n.d. & 9.58 & 1.14 & 1.33 & n.d. & n.d. & n.d. & n.d. & n.d. & n.d. & 5.45 & 3.13 & 19.85 \\
\hline
\end{tabular}




\begin{tabular}{|c|c|c|c|c|c|c|c|c|c|c|c|c|c|}
\hline $\begin{array}{l}\text { Concentration } \\
\text { (ng/L) }\end{array}$ & PFHpS & PFOS & PFNS & PFDS & PFDoDS & FOSA & FOSAA & NEtFOSAA & NMeFOSAA & NMeFOSA & NEtFOSA & 10:2 FTS & 8:2 FTS \\
\hline 29/03/2017 & 1.18 & 34.43 & n.d. & n.d. & n.d. & n.d. & $<\mathrm{LOQ}$ & n.d. & n.d. & n.d. & n.d. & n.d. & n.d. \\
\hline $31 / 03 / 2017$ & 0.54 & 12.65 & n.d. & n.d. & n.d. & n.d. & $<\mathrm{LOQ}$ & n.d. & n.d. & n.d. & n.d. & n.d. & n.d. \\
\hline 01/04/2017 & 0.48 & 9.80 & n.d. & n.d. & n.d. & n.d. & $<\mathrm{LOQ}$ & n.d. & n.d. & n.d. & n.d. & n.d. & n.d. \\
\hline 02/04/2017 & $<\mathrm{LOQ}$ & 3.41 & n.d. & n.d. & n.d. & n.d. & n.d. & n.d. & n.d. & n.d. & n.d. & n.d. & n.d. \\
\hline 03/04/2017 & 0.24 & 4.42 & n.d. & n.d. & $<\mathrm{LOQ}$ & n.d. & n.d. & n.d. & n.d. & n.d. & n.d. & n.d. & n.d. \\
\hline $04 / 04 / 2017$ & $<\mathrm{LOQ}$ & 5.04 & n.d. & n.d. & n.d. & n.d. & n.d. & n.d. & n.d. & n.d. & n.d. & n.d. & n.d. \\
\hline 05/04/2017 & $<\mathrm{LOQ}$ & 4.70 & n.d. & n.d. & n.d. & n.d. & n.d. & n.d. & n.d. & n.d. & n.d. & n.d. & n.d. \\
\hline 06/04/2017 & $<\mathrm{LOQ}$ & 4.12 & n.d. & n.d. & n.d. & $<\mathrm{LOQ}$ & n.d. & n.d. & n.d. & n.d. & n.d. & n.d. & n.d. \\
\hline $07 / 04 / 2017$ & $<\mathrm{LOQ}$ & 4.07 & n.d. & n.d. & n.d. & n.d. & n.d. & n.d. & n.d. & n.d. & n.d. & n.d. & n.d. \\
\hline 09/04/2017 & $<\mathrm{LOQ}$ & 4.62 & n.d. & n.d. & n.d. & n.d. & n.d. & n.d. & n.d. & n.d. & n.d. & n.d. & n.d. \\
\hline $10 / 04 / 2017$ & $<\mathrm{LOQ}$ & 4.89 & n.d. & n.d. & n.d. & n.d. & n.d. & n.d. & n.d. & n.d. & n.d. & n.d. & n.d. \\
\hline $12 / 04 / 2017$ & $<\mathrm{LOQ}$ & 4.77 & n.d. & n.d. & n.d. & $<L O Q$ & n.d. & n.d. & n.d. & n.d. & n.d. & n.d. & n.d. \\
\hline $14 / 04 / 2017$ & $<\mathrm{LOQ}$ & 5.44 & n.d. & n.d. & n.d. & n.d. & n.d. & n.d. & n.d. & n.d. & n.d. & n.d. & n.d. \\
\hline $15 / 04 / 2017$ & 0.20 & 5.99 & n.d. & n.d. & n.d. & n.d. & n.d. & n.d. & n.d. & n.d. & n.d. & n.d. & n.d. \\
\hline $17 / 04 / 2017$ & 0.20 & 5.78 & n.d. & n.d. & n.d. & n.d. & n.d. & n.d. & n.d. & n.d. & n.d. & n.d. & n.d. \\
\hline 19/04/2017 & 0.21 & 5.24 & n.d. & n.d. & n.d. & $<L O Q$ & n.d. & n.d. & n.d. & n.d. & n.d. & n.d. & n.d. \\
\hline 26/04/2017 & 0.21 & 6.55 & n.d. & n.d. & n.d. & n.d. & n.d. & n.d. & n.d. & n.d. & n.d. & n.d. & n.d. \\
\hline 03/05/2017 & 0.20 & 7.34 & n.d. & n.d. & n.d. & n.d. & n.d. & n.d. & n.d. & n.d. & n.d. & n.d. & n.d. \\
\hline $11 / 05 / 2017$ & 0.18 & 5.85 & n.d. & n.d. & n.d. & n.d. & n.d. & n.d. & n.d. & n.d. & n.d. & n.d. & n.d. \\
\hline $17 / 05 / 2017$ & 0.18 & 6.23 & n.d. & n.d. & n.d. & $<L O Q$ & n.d. & n.d. & n.d. & n.d. & $<\mathrm{LOQ}$ & n.d. & n.d. \\
\hline 24/05/2017 & 0.21 & 7.14 & n.d. & n.d. & n.d. & $<\mathrm{LOQ}$ & n.d. & n.d. & n.d. & n.d. & n.d. & n.d. & n.d. \\
\hline $31 / 05 / 2017$ & 0.26 & 7.64 & n.d. & n.d. & n.d. & n.d. & n.d. & n.d. & n.d. & n.d. & n.d. & n.d. & n.d. \\
\hline 07/06/2017 & 0.26 & 6.31 & n.d. & n.d. & n.d. & n.d. & n.d. & n.d. & n.d. & n.d. & n.d. & n.d. & n.d. \\
\hline $21 / 06 / 2017$ & 1.40 & 21.08 & n.d. & n.d. & n.d. & $<L O Q$ & n.d. & n.d. & n.d. & n.d. & n.d. & n.d. & n.d. \\
\hline 29/06/2017 & 0.25 & 7.55 & n.d. & n.d. & n.d. & $<\mathrm{LOQ}$ & n.d. & n.d. & $<\mathrm{LOQ}$ & n.d. & n.d. & n.d. & n.d. \\
\hline 05/07/2017 & 0.28 & 9.35 & n.d. & n.d. & n.d. & $<\mathrm{LOQ}$ & n.d. & n.d. & n.d. & 0.68 & n.d. & n.d. & n.d. \\
\hline $19 / 07 / 2017$ & 0.34 & 7.99 & n.d. & n.d. & n.d. & $<\mathrm{LOQ}$ & n.d. & $<\mathrm{LOQ}$ & n.d. & n.d. & n.d. & n.d. & n.d. \\
\hline 27/07/2017 & 0.34 & 8.15 & n.d. & n.d. & n.d. & $<L O Q$ & n.d. & n.d. & n.d. & n.d. & n.d. & n.d. & n.d. \\
\hline 02/08/2017 & 0.35 & 8.96 & n.d. & n.d. & n.d. & $<\mathrm{LOQ}$ & n.d. & n.d. & n.d. & n.d. & n.d. & n.d. & n.d. \\
\hline 30/08/2017 & 0.38 & 9.26 & n.d. & n.d. & $<\mathrm{LOQ}$ & 0.63 & n.d. & 0.53 & n.d. & n.d. & n.d. & n.d. & n.d. \\
\hline $17 / 09 / 2017$ & 0.34 & 7.52 & n.d. & n.d. & n.d. & $<\mathrm{LOQ}$ & n.d. & n.d. & n.d. & n.d. & n.d. & n.d. & n.d. \\
\hline $27 / 09 / 2017$ & 0.38 & 8.31 & n.d. & n.d. & n.d. & $<\mathrm{LOQ}$ & n.d. & n.d. & n.d. & n.d. & n.d. & n.d. & n.d. \\
\hline 04/10/2017 & 0.33 & 7.25 & n.d. & n.d. & n.d. & $<\mathrm{LOQ}$ & n.d. & n.d. & $<$ LOQ & n.d. & n.d. & n.d. & n.d. \\
\hline $18 / 10 / 2017$ & 0.47 & 10.35 & n.d. & n.d. & n.d. & 0.45 & $<\mathrm{LOQ}$ & $<\mathrm{LOQ}$ & 0.32 & n.d. & n.d. & n.d. & n.d. \\
\hline 01/11/2017 & 0.55 & 14.61 & n.d. & n.d. & n.d. & 0.52 & n.d. & $<\mathrm{LOQ}$ & 0.45 & n.d. & n.d. & n.d. & n.d. \\
\hline $16 / 11 / 2017$ & 0.47 & 14.03 & n.d. & n.d. & n.d. & $<\mathrm{LOQ}$ & n.d. & n.d. & n.d. & n.d. & n.d. & n.d. & n.d. \\
\hline $12 / 12 / 2017$ & 0.85 & 26.49 & n.d. & n.d. & n.d. & n.d. & n.d. & n.d. & n.d. & n.d. & n.d. & n.d. & n.d. \\
\hline
\end{tabular}




\begin{tabular}{|c|c|c|c|c|c|c|c|c|c|c|c|}
\hline $\begin{array}{l}\text { Concentration } \\
\text { (ng/L) }\end{array}$ & $6: 2 \mathrm{FTS}$ & 4:2FTS & NMeFOSE & NetFOSE & PFECHS & 6:2PAP & 8:2PAP & SamPAP & 6:2DiPAP & 8:2DiPAP & 6:28:2DiPAP \\
\hline 29/03/2017 & 0.47 & n.d. & n.d. & n.d. & $<\mathrm{LOQ}$ & n.d. & n.d. & n.d. & n.d. & n.d. & n.d. \\
\hline $31 / 03 / 2017$ & 0.77 & n.d. & n.d. & n.d. & n.d. & n.d. & n.d. & n.d. & 689.88 & n.d. & n.d. \\
\hline $01 / 04 / 2017$ & 0.86 & n.d. & n.d. & n.d. & n.d. & n.d. & n.d. & n.d. & n.d. & n.d. & n.d. \\
\hline $02 / 04 / 2017$ & $<\mathrm{LOQ}$ & n.d. & n.d. & n.d. & n.d. & n.d. & n.d. & n.d. & n.d. & n.d. & n.d. \\
\hline 03/04/2017 & $<\mathrm{LOQ}$ & n.d. & n.d. & n.d. & n.d. & n.d. & n.d. & n.d. & n.d. & n.d. & n.d. \\
\hline 04/04/2017 & $<L O Q$ & n.d. & n.d. & n.d. & n.d. & n.d. & n.d. & n.d. & n.d. & n.d. & n.d. \\
\hline 05/04/2017 & $<\mathrm{LOQ}$ & n.d. & n.d. & n.d. & n.d. & n.d. & n.d. & n.d. & n.d. & n.d. & n.d. \\
\hline 06/04/2017 & $<\mathrm{LOQ}$ & n.d. & n.d. & n.d. & n.d. & n.d. & n.d. & n.d. & n.d. & n.d. & n.d. \\
\hline 07/04/2017 & $<\mathrm{LOQ}$ & n.d. & n.d. & n.d. & n.d. & n.d. & n.d. & n.d. & n.d. & n.d. & n.d. \\
\hline 09/04/2017 & $<\mathrm{LOQ}$ & n.d. & $<\mathrm{LOQ}$ & n.d. & n.d. & n.d. & n.d. & n.d. & n.d. & n.d. & n.d. \\
\hline $10 / 04 / 2017$ & $<L O Q$ & n.d. & n.d. & n.d. & n.d. & n.d. & n.d. & n.d. & n.d. & n.d. & n.d. \\
\hline $12 / 04 / 2017$ & $<\mathrm{LOQ}$ & n.d. & n.d. & n.d. & n.d. & n.d. & n.d. & n.d. & n.d. & n.d. & n.d. \\
\hline $14 / 04 / 2017$ & $<\mathrm{LOQ}$ & n.d. & n.d. & n.d. & n.d. & n.d. & n.d. & n.d. & 38.48 & n.d. & n.d. \\
\hline $15 / 04 / 2017$ & $<\mathrm{LOQ}$ & n.d. & n.d. & n.d. & n.d. & n.d. & n.d. & n.d. & 33.43 & n.d. & n.d. \\
\hline $17 / 04 / 2017$ & $<\mathrm{LOQ}$ & n.d. & n.d. & $<\mathrm{LOQ}$ & n.d. & n.d. & n.d. & n.d. & 63.28 & n.d. & n.d. \\
\hline $19 / 04 / 2017$ & $<L O Q$ & n.d. & n.d. & n.d. & n.d. & n.d. & n.d. & n.d. & n.d. & n.d. & n.d. \\
\hline $26 / 04 / 2017$ & $<\mathrm{LOQ}$ & n.d. & n.d. & n.d. & n.d. & n.d. & n.d. & n.d. & n.d. & n.d. & n.d. \\
\hline $03 / 05 / 2017$ & $<\mathrm{LOQ}$ & n.d. & $<\mathrm{LOQ}$ & 0.80 & n.d. & n.d. & n.d. & n.d. & 79.83 & n.d. & n.d. \\
\hline $11 / 05 / 2017$ & $<\mathrm{LOQ}$ & n.d. & n.d. & n.d. & n.d. & n.d. & n.d. & n.d. & n.d. & n.d. & n.d. \\
\hline $17 / 05 / 2017$ & $<\mathrm{LOQ}$ & n.d. & n.d. & $<\mathrm{LOQ}$ & n.d. & n.d. & n.d. & n.d. & n.d. & n.d. & n.d. \\
\hline $24 / 05 / 2017$ & $<\mathrm{LOQ}$ & n.d. & n.d. & n.d. & n.d. & n.d. & n.d. & n.d. & n.d. & n.d. & n.d. \\
\hline $31 / 05 / 2017$ & $<\mathrm{LOQ}$ & n.d. & n.d. & n.d. & n.d. & n.d. & n.d. & n.d. & 401.53 & n.d. & n.d. \\
\hline 07/06/2017 & $<\mathrm{LOQ}$ & n.d. & n.d. & n.d. & n.d. & n.d. & n.d. & n.d. & n.d. & n.d. & n.d. \\
\hline $21 / 06 / 2017$ & 0.36 & n.d. & $<\mathrm{LOQ}$ & n.d. & n.d. & n.d. & n.d. & n.d. & n.d. & n.d. & n.d. \\
\hline 29/06/2017 & $<\mathrm{LOQ}$ & n.d. & n.d. & $<\mathrm{LOQ}$ & n.d. & n.d. & n.d. & n.d. & 79.57 & n.d. & n.d. \\
\hline 05/07/2017 & $<\mathrm{LOQ}$ & n.d. & n.d. & $<\mathrm{LOQ}$ & n.d. & n.d. & n.d. & n.d. & n.d. & n.d. & n.d. \\
\hline 19/07/2017 & $<\mathrm{LOQ}$ & n.d. & n.d. & $<\mathrm{LOQ}$ & n.d. & n.d. & n.d. & n.d. & n.d. & n.d. & n.d. \\
\hline 27/07/2017 & $<L O Q$ & n.d. & n.d. & n.d. & n.d. & n.d. & n.d. & n.d. & n.d. & n.d. & n.d. \\
\hline 02/08/2017 & $<\mathrm{LOQ}$ & n.d. & $<\mathrm{LOQ}$ & $<\mathrm{LOQ}$ & n.d. & n.d. & n.d. & n.d. & n.d. & n.d. & n.d. \\
\hline $30 / 08 / 2017$ & $<\mathrm{LOQ}$ & n.d. & $<\mathrm{LOQ}$ & n.d. & n.d. & n.d. & n.d. & n.d. & n.d. & n.d. & n.d. \\
\hline $17 / 09 / 2017$ & 0.54 & n.d. & n.d. & $<\mathrm{LOQ}$ & $<\mathrm{LOQ}$ & n.d. & n.d. & n.d. & n.d. & n.d. & n.d. \\
\hline 27/09/2017 & 0.33 & n.d. & $<\mathrm{LOQ}$ & n.d. & n.d. & n.d. & n.d. & n.d. & n.d. & n.d. & n.d. \\
\hline 04/10/2017 & $<\mathrm{LOQ}$ & n.d. & n.d. & n.d. & $<\mathrm{LOQ}$ & n.d. & n.d. & n.d. & n.d. & n.d. & n.d. \\
\hline $18 / 10 / 2017$ & 0.30 & n.d. & n.d. & n.d. & $<\mathrm{LOQ}$ & n.d. & n.d. & n.d. & n.d. & n.d. & n.d. \\
\hline $01 / 11 / 2017$ & $<\mathrm{LOQ}$ & n.d. & n.d. & n.d. & $<\mathrm{LOQ}$ & n.d. & n.d. & n.d. & n.d. & n.d. & n.d. \\
\hline $16 / 11 / 2017$ & $<L O Q$ & n.d. & $<\mathrm{LOQ}$ & $<\mathrm{LOQ}$ & n.d. & n.d. & n.d. & n.d. & n.d. & n.d. & n.d. \\
\hline $12 / 12 / 2017$ & 0.35 & n.d. & n.d. & n.d. & n.d. & n.d. & n.d. & n.d. & n.d. & n.d. & n.d. \\
\hline
\end{tabular}


Table S-11. PPCP and pesticide concentrations in surface water samples.

\begin{tabular}{|c|c|c|c|c|c|c|c|c|c|c|c|c|}
\hline $\begin{array}{l}\text { Concentra- } \\
\text { tion (ng/L) }\end{array}$ & Salicylic acid & Acesulfame & MCPA & Ibuprofen & Mecoprop & $2,4-D$ & Dicamba & $2,4-D B$ & $2,4,5-\mathrm{T}$ & Triclopyr & Bromoxynil & Triclosan \\
\hline 29/03/2017 & n.d. & 321.52 & 31.30 & 22.55 & n.d. & 36.87 & 97.79 & n.d. & n.d. & 1.99 & n.d. & n.d. \\
\hline $31 / 03 / 2017$ & n.d. & n.d. & 26.51 & n.d. & n.d. & 23.45 & 215.23 & n.d. & n.d. & $<\mathrm{LOQ}$ & n.d. & n.d. \\
\hline 01/04/2017 & n.d. & n.d. & 20.88 & n.d. & n.d. & 17.30 & 161.20 & n.d. & n.d. & n.d. & n.d. & n.d. \\
\hline 02/04/2017 & n.d. & n.d. & 11.55 & n.d. & n.d. & 32.98 & 160.50 & n.d. & n.d. & $<\mathrm{LOQ}$ & n.d. & n.d. \\
\hline 03/04/2017 & n.d. & n.d. & 25.72 & n.d. & n.d. & 31.84 & 145.31 & n.d. & n.d. & $<\mathrm{LOQ}$ & n.d. & n.d. \\
\hline 04/04/2017 & n.d. & n.d. & 11.83 & n.d. & n.d. & 35.32 & 124.11 & n.d. & n.d. & $<\mathrm{LOQ}$ & n.d. & 2.26 \\
\hline 05/04/2017 & n.d. & n.d. & 10.50 & n.d. & n.d. & 36.89 & 133.78 & n.d. & n.d. & 1.60 & n.d. & 1.79 \\
\hline 06/04/2017 & n.d. & n.d. & 10.49 & n.d. & n.d. & 36.58 & 166.72 & n.d. & n.d. & 1.73 & n.d. & 1.68 \\
\hline 07/04/2017 & n.d. & n.d. & 11.65 & n.d. & n.d. & 32.16 & 168.99 & n.d. & n.d. & 1.52 & n.d. & 2.35 \\
\hline 09/04/2017 & n.d. & n.d. & 9.28 & n.d. & n.d. & 32.80 & 136.92 & n.d. & n.d. & 1.78 & n.d. & n.d. \\
\hline 10/04/2017 & n.d. & n.d. & 8.90 & n.d. & n.d. & 33.09 & 148.45 & n.d. & n.d. & 1.99 & n.d. & 1.25 \\
\hline $12 / 04 / 2017$ & n.d. & n.d. & 8.09 & n.d. & n.d. & 31.98 & 132.57 & 17.00 & n.d. & $<\mathrm{LOQ}$ & n.d. & n.d. \\
\hline 14/04/2017 & n.d. & n.d. & 8.93 & n.d. & n.d. & 30.13 & 80.76 & n.d. & n.d. & 1.71 & n.d. & n.d. \\
\hline 15/04/2017 & n.d. & n.d. & 9.26 & n.d. & n.d. & 28.82 & 133.82 & n.d. & n.d. & 1.55 & n.d. & n.d. \\
\hline $17 / 04 / 2017$ & n.d. & n.d. & 9.81 & n.d. & n.d. & 25.81 & 110.69 & n.d. & n.d. & $<\mathrm{LOQ}$ & n.d. & n.d. \\
\hline 19/04/2017 & n.d. & n.d. & 8.90 & n.d. & n.d. & 24.39 & 118.16 & n.d. & n.d. & $<\mathrm{LOQ}$ & n.d. & n.d. \\
\hline 26/04/2017 & n.d. & n.d. & 8.79 & n.d. & n.d. & 21.76 & 97.25 & n.d. & n.d. & $<\mathrm{LOQ}$ & n.d. & n.d. \\
\hline 03/05/2017 & n.d. & 401.21 & 9.11 & n.d. & n.d. & 17.41 & 78.20 & n.d. & n.d. & 1.53 & n.d. & 1.53 \\
\hline $11 / 05 / 2017$ & n.d. & n.d. & 8.59 & n.d. & n.d. & 14.32 & 88.99 & n.d. & n.d. & 1.88 & n.d. & n.d. \\
\hline $17 / 05 / 2017$ & n.d. & n.d. & 8.98 & n.d. & n.d. & 12.22 & 70.50 & n.d. & n.d. & 1.73 & n.d. & n.d. \\
\hline 24/05/2017 & n.d. & n.d. & 10.09 & n.d. & n.d. & 11.82 & 79.93 & n.d. & n.d. & 1.92 & n.d. & n.d. \\
\hline $31 / 05 / 2017$ & n.d. & n.d. & 12.43 & n.d. & n.d. & 21.62 & 55.64 & n.d. & n.d. & 2.03 & n.d. & n.d. \\
\hline 07/06/2017 & n.d. & n.d. & 12.51 & n.d. & n.d. & 19.53 & 77.38 & n.d. & n.d. & 4.22 & n.d. & n.d. \\
\hline $21 / 06 / 2017$ & n.d. & n.d. & 15.97 & n.d. & n.d. & 39.68 & 91.75 & n.d. & n.d. & 4.75 & n.d. & n.d. \\
\hline 29/06/2017 & n.d. & n.d. & 16.15 & n.d. & n.d. & 39.13 & 77.83 & n.d. & n.d. & 4.33 & n.d. & n.d. \\
\hline 05/07/2017 & n.d. & n.d. & 16.41 & n.d. & n.d. & 36.49 & 87.53 & n.d. & n.d. & 4.47 & n.d. & n.d. \\
\hline 19/07/2017 & n.d. & 434.34 & 29.52 & n.d. & n.d. & 41.98 & 103.60 & n.d. & n.d. & 4.48 & n.d. & n.d. \\
\hline 27/07/2017 & n.d. & n.d. & 21.74 & n.d. & n.d. & 34.09 & 68.49 & n.d. & n.d. & 3.62 & n.d. & n.d. \\
\hline 02/08/2017 & n.d. & n.d. & 27.13 & n.d. & n.d. & 40.58 & 57.68 & n.d. & n.d. & 2.84 & n.d. & n.d. \\
\hline $30 / 08 / 2017$ & n.d. & 314.31 & 23.40 & n.d. & n.d. & 28.39 & 80.56 & n.d. & n.d. & 4.07 & n.d. & n.d. \\
\hline 17/09/2017 & n.d. & 883.25 & 62.51 & n.d. & n.d. & 207.40 & 127.24 & n.d. & n.d. & 5.70 & n.d. & n.d. \\
\hline 27/09/2017 & n.d. & 404.67 & 57.63 & n.d. & n.d. & 254.33 & 114.07 & n.d. & n.d. & 4.57 & n.d. & n.d. \\
\hline 04/10/2017 & n.d. & n.d. & 6.81 & n.d. & n.d. & 23.16 & 58.58 & n.d. & n.d. & 3.29 & n.d. & n.d. \\
\hline 18/10/2017 & n.d. & n.d. & 30.01 & n.d. & $<\mathrm{LOQ}$ & 32.55 & 117.86 & n.d. & n.d. & 3.44 & n.d. & n.d. \\
\hline 01/11/2017 & n.d. & 700.45 & 27.62 & n.d. & n.d. & 27.30 & 50.38 & n.d. & n.d. & $<\mathrm{LOQ}$ & n.d. & n.d. \\
\hline $16 / 11 / 2017$ & 198.99 & n.d. & 26.14 & n.d. & n.d. & 17.75 & 73.34 & n.d. & n.d. & 2.84 & n.d. & n.d. \\
\hline $12 / 12 / 2017$ & n.d. & n.d. & 39.47 & n.d. & n.d. & 38.94 & 81.42 & n.d. & n.d. & 2.28 & n.d. & n.d. \\
\hline
\end{tabular}




\begin{tabular}{|c|c|c|c|c|c|c|c|c|c|c|c|}
\hline $\begin{array}{l}\text { Concentra- } \\
\text { tion (ng/L) }\end{array}$ & $\begin{array}{r}\text { Hydrochloro- } \\
\text { thiazide }\end{array}$ & Furosemide & $\begin{array}{r}\text { Diketoni- } \\
\text { trile }\end{array}$ & Haloxyfop - & Paracetamol & $\begin{array}{r}3,4 \mathrm{DiCl} \\
\text { Aniline }\end{array}$ & $\begin{array}{r}\text { Metho- } \\
\text { myl }\end{array}$ & Nicotine & Gabapentin & $\begin{array}{r}\text { Desisopropyl } \\
\text { Atrazine }\end{array}$ & Cotinine \\
\hline 29/03/2017 & 10.22 & n.d. & 0.55 & 3.69 & n.d. & n.d. & 0.39 & n.d. & 46.14 & 8.16 & 6.97 \\
\hline $31 / 03 / 2017$ & n.d. & n.d. & n.d. & n.d. & 84.32 & n.d. & 0.75 & n.d. & n.d. & 1.50 & 1.73 \\
\hline 01/04/2017 & n.d. & n.d. & n.d. & n.d. & n.d. & n.d. & 0.30 & n.d. & n.d. & 2.10 & 0.97 \\
\hline 02/04/2017 & n.d. & n.d. & n.d. & 3.80 & n.d. & n.d. & 180.33 & n.d. & n.d. & 0.95 & 0.84 \\
\hline 03/04/2017 & 2.23 & n.d. & n.d. & 2.58 & n.d. & n.d. & 46.17 & n.d. & 18.46 & 1.72 & 1.25 \\
\hline 04/04/2017 & 2.17 & n.d. & n.d. & 3.32 & n.d. & n.d. & 29.46 & n.d. & 14.04 & 1.70 & 1.17 \\
\hline 05/04/2017 & 2.69 & n.d. & n.d. & 4.20 & n.d. & n.d. & 27.23 & n.d. & 10.24 & 1.31 & 1.53 \\
\hline 06/04/2017 & 3.39 & n.d. & n.d. & 4.43 & n.d. & n.d. & 26.15 & n.d. & 11.07 & 1.31 & 1.33 \\
\hline 07/04/2017 & 3.23 & n.d. & n.d. & 4.25 & n.d. & n.d. & 18.99 & n.d. & 9.06 & 1.66 & 0.98 \\
\hline 09/04/2017 & 4.99 & n.d. & n.d. & 4.69 & n.d. & n.d. & 14.30 & n.d. & 8.96 & 1.28 & 1.38 \\
\hline 10/04/2017 & 5.87 & n.d. & $<\mathrm{LOQ}$ & 5.33 & n.d. & n.d. & 14.67 & n.d. & 9.96 & 1.38 & 1.26 \\
\hline $12 / 04 / 2017$ & 5.45 & n.d. & n.d. & 4.48 & n.d. & n.d. & 11.62 & n.d. & 7.82 & 1.38 & 1.18 \\
\hline $14 / 04 / 2017$ & 6.95 & n.d. & $<\mathrm{LOQ}$ & 5.42 & n.d. & n.d. & 12.98 & n.d. & 11.26 & 1.39 & 1.44 \\
\hline $15 / 04 / 2017$ & 6.19 & n.d. & $<\mathrm{LOQ}$ & 4.69 & n.d. & n.d. & 9.66 & n.d. & 11.36 & 1.70 & 1.40 \\
\hline 17/04/2017 & 6.59 & n.d. & $<\mathrm{LOQ}$ & 4.49 & n.d. & n.d. & 9.20 & n.d. & 11.93 & 1.39 & 1.49 \\
\hline 19/04/2017 & 8.68 & n.d. & $<L O Q$ & 4.10 & n.d. & n.d. & 9.81 & n.d. & 17.31 & 1.38 & 1.53 \\
\hline 26/04/2017 & 11.91 & n.d. & $<\mathrm{LOQ}$ & 3.88 & n.d. & n.d. & 9.42 & n.d. & 17.74 & 1.53 & 1.77 \\
\hline 03/05/2017 & 12.15 & n.d. & $<\mathrm{LOQ}$ & 3.73 & n.d. & n.d. & 8.13 & n.d. & 19.73 & 1.73 & 1.94 \\
\hline $11 / 05 / 2017$ & 16.62 & n.d. & 0.38 & 4.67 & n.d. & n.d. & 7.83 & n.d. & 25.72 & 1.61 & 2.42 \\
\hline $17 / 05 / 2017$ & 14.96 & n.d. & 0.38 & 4.83 & n.d. & n.d. & 6.78 & n.d. & 27.41 & 1.60 & 2.57 \\
\hline 24/05/2017 & 16.56 & n.d. & 0.48 & 5.50 & 24.11 & n.d. & 7.30 & n.d. & 33.53 & 1.80 & 3.29 \\
\hline $31 / 05 / 2017$ & 16.37 & n.d. & $<\mathrm{LOQ}$ & 4.53 & 5.23 & n.d. & 4.64 & n.d. & 32.68 & 1.60 & 3.06 \\
\hline 07/06/2017 & 19.17 & n.d. & 0.35 & 4.32 & n.d. & n.d. & 5.95 & n.d. & 36.58 & 1.85 & 3.66 \\
\hline $21 / 06 / 2017$ & 19.22 & n.d. & 0.39 & 5.95 & 7.00 & n.d. & 4.54 & n.d. & 38.23 & 1.64 & 4.66 \\
\hline 29/06/2017 & 16.23 & n.d. & 0.36 & 4.92 & n.d. & n.d. & 3.36 & n.d. & 37.58 & 1.92 & 5.14 \\
\hline 05/07/2017 & 20.84 & n.d. & 0.52 & 4.62 & n.d. & n.d. & 3.23 & n.d. & 42.15 & 1.77 & 5.44 \\
\hline 19/07/2017 & 26.13 & n.d. & 0.57 & 5.26 & 11.99 & n.d. & 2.92 & n.d. & 52.62 & 2.23 & 6.36 \\
\hline $27 / 07 / 2017$ & 18.69 & n.d. & 0.42 & 5.62 & n.d. & n.d. & 2.00 & n.d. & 47.74 & 1.88 & 5.75 \\
\hline 02/08/2017 & 24.44 & n.d. & 0.35 & 4.83 & n.d. & n.d. & 1.97 & n.d. & 44.17 & 2.12 & 6.42 \\
\hline $30 / 08 / 2017$ & 14.92 & n.d. & 0.67 & 4.27 & n.d. & n.d. & 1.04 & n.d. & 46.21 & 2.26 & 8.83 \\
\hline 17/09/2017 & 19.20 & n.d. & 1.42 & 16.29 & n.d. & n.d. & 1.84 & n.d. & 54.87 & 2.61 & 8.91 \\
\hline 27/09/2017 & 13.24 & n.d. & 1.99 & 17.87 & 9.09 & n.d. & 0.90 & n.d. & 38.52 & 2.13 & 11.96 \\
\hline 04/10/2017 & 5.63 & n.d. & 1.92 & 15.99 & n.d. & n.d. & 0.61 & n.d. & 40.96 & 1.57 & 5.97 \\
\hline 18/10/2017 & 13.24 & n.d. & 1.17 & 11.02 & n.d. & n.d. & 0.65 & n.d. & 57.73 & 3.64 & 9.15 \\
\hline 01/11/2017 & 7.97 & n.d. & $<\mathrm{LOQ}$ & 4.13 & n.d. & n.d. & 2.03 & n.d. & 32.89 & 2.98 & 7.69 \\
\hline $16 / 11 / 2017$ & 7.98 & n.d. & 0.66 & 3.43 & n.d. & 0.82 & 3.28 & n.d. & 33.06 & 3.04 & 7.01 \\
\hline $12 / 12 / 2017$ & 7.20 & n.d. & 0.68 & 3.78 & n.d. & n.d. & 1.48 & n.d. & 46.28 & 3.91 & 6.30 \\
\hline
\end{tabular}




\begin{tabular}{|c|c|c|c|c|c|c|c|c|c|c|c|}
\hline $\begin{array}{l}\text { Concentra- } \\
\text { tion (ng/L) }\end{array}$ & Paraxanthine & $\begin{array}{r}\text { Simazine } \\
\text { hydroxy }\end{array}$ & $\begin{array}{l}\text { Desethyl } \\
\text { Atrazine }\end{array}$ & Clopyralid & 5 HIAA & DEET & $\begin{array}{r}\text { Hydroxyco- } \\
\text { tinine }\end{array}$ & Caffeine & $\begin{array}{r}\text { Ametrynhy- } \\
\text { droxy }\end{array}$ & Pyrimethanil & $\begin{array}{r}\text { Terbuthylazine } \\
\text { des ethyl }\end{array}$ \\
\hline 29/03/2017 & n.d. & 8.59 & 3.03 & n.d. & n.d. & 12.72 & 4.51 & 12.22 & 5.77 & n.d. & 0.61 \\
\hline $31 / 03 / 2017$ & 3.03 & $<\mathrm{LOQ}$ & n.d. & n.d. & n.d. & 8.40 & 3.60 & 9.28 & $<\mathrm{LOQ}$ & n.d. & n.d. \\
\hline 01/04/2017 & 3.18 & 1.44 & 0.27 & n.d. & n.d. & 5.33 & n.d. & 4.78 & 1.42 & n.d. & n.d. \\
\hline 02/04/2017 & n.d. & n.d. & 0.89 & n.d. & n.d. & 5.33 & n.d. & 3.72 & 2.66 & n.d. & n.d. \\
\hline 03/04/2017 & n.d. & $<\mathrm{LOQ}$ & 0.71 & n.d. & n.d. & 7.35 & 1.35 & 7.12 & 2.87 & n.d. & n.d. \\
\hline 04/04/2017 & 2.07 & $<\mathrm{LOQ}$ & 1.00 & n.d. & n.d. & 7.76 & 1.39 & 9.40 & 3.06 & n.d. & n.d. \\
\hline 05/04/2017 & n.d. & $<\mathrm{LOQ}$ & 1.06 & n.d. & n.d. & 8.31 & 1.30 & 7.34 & 3.46 & n.d. & n.d. \\
\hline 06/04/2017 & n.d. & $<\mathrm{LOQ}$ & 1.09 & n.d. & n.d. & 8.90 & 1.54 & 10.87 & 3.32 & n.d. & n.d. \\
\hline 07/04/2017 & n.d. & $<\mathrm{LOQ}$ & 0.98 & n.d. & n.d. & 7.08 & n.d. & 2.86 & 3.24 & n.d. & n.d. \\
\hline 09/04/2017 & n.d. & $<\mathrm{LOQ}$ & 1.21 & n.d. & n.d. & 11.16 & 1.43 & 9.70 & 3.49 & n.d. & n.d. \\
\hline $10 / 04 / 2017$ & n.d. & $<\mathrm{LOQ}$ & 1.23 & n.d. & n.d. & 9.22 & 1.49 & 6.32 & 3.36 & n.d. & n.d. \\
\hline $12 / 04 / 2017$ & n.d. & $<\mathrm{LOQ}$ & 1.30 & n.d. & n.d. & 12.40 & 1.33 & 5.26 & 3.38 & n.d. & n.d. \\
\hline $14 / 04 / 2017$ & n.d. & $<\mathrm{LOQ}$ & 1.29 & n.d. & n.d. & 8.33 & 1.79 & 19.74 & 3.01 & n.d. & n.d. \\
\hline $15 / 04 / 2017$ & n.d. & $<\mathrm{LOQ}$ & 1.46 & n.d. & n.d. & 12.70 & 1.56 & 6.70 & 3.09 & n.d. & n.d. \\
\hline $17 / 04 / 2017$ & n.d. & 1.24 & 1.20 & n.d. & n.d. & 17.41 & 1.51 & 7.26 & 3.68 & n.d. & n.d. \\
\hline 19/04/2017 & n.d. & $<\mathrm{LOQ}$ & 1.24 & n.d. & n.d. & 15.11 & 1.71 & 19.74 & 3.04 & n.d. & n.d. \\
\hline $26 / 04 / 2017$ & n.d. & $<\mathrm{LOQ}$ & 1.45 & n.d. & n.d. & 13.32 & 2.08 & 7.32 & 3.30 & n.d. & n.d. \\
\hline 03/05/2017 & n.d. & $<\mathrm{LOQ}$ & 1.45 & n.d. & n.d. & 37.66 & 2.25 & 6.64 & 3.63 & n.d. & n.d. \\
\hline $11 / 05 / 2017$ & n.d. & 1.34 & 1.43 & n.d. & n.d. & 14.87 & 2.88 & 6.52 & 4.26 & n.d. & n.d. \\
\hline $17 / 05 / 2017$ & 4.47 & 1.22 & 1.60 & n.d. & n.d. & 28.61 & 2.84 & 22.62 & 3.85 & n.d. & n.d. \\
\hline $24 / 05 / 2017$ & n.d. & 1.38 & 1.68 & n.d. & n.d. & 46.77 & 3.56 & 9.26 & 4.46 & n.d. & n.d. \\
\hline $31 / 05 / 2017$ & 4.29 & 1.24 & 1.63 & n.d. & n.d. & 38.52 & 3.11 & 7.69 & 3.70 & n.d. & n.d. \\
\hline 07/06/2017 & n.d. & 1.46 & 1.66 & n.d. & n.d. & 36.81 & 4.04 & 10.82 & 4.17 & n.d. & n.d. \\
\hline $21 / 06 / 2017$ & n.d. & 1.52 & 1.34 & n.d. & n.d. & 31.34 & 4.30 & 16.01 & 4.20 & n.d. & n.d. \\
\hline 29/06/2017 & n.d. & 1.86 & 1.39 & n.d. & n.d. & 37.65 & 4.50 & 23.51 & 4.83 & n.d. & n.d. \\
\hline 05/07/2017 & 3.09 & 1.80 & 1.33 & n.d. & n.d. & 40.37 & 4.92 & 15.64 & 5.03 & n.d. & n.d. \\
\hline 19/07/2017 & n.d. & 1.87 & 1.68 & n.d. & n.d. & 44.03 & 5.94 & 17.73 & 4.57 & n.d. & n.d. \\
\hline $27 / 07 / 2017$ & n.d. & 1.46 & 1.48 & n.d. & n.d. & 46.47 & 4.78 & 11.06 & 3.54 & n.d. & 0.31 \\
\hline 02/08/2017 & n.d. & $<\mathrm{LOQ}$ & 1.80 & n.d. & n.d. & 64.02 & 5.61 & 13.46 & 2.77 & n.d. & 0.34 \\
\hline $30 / 08 / 2017$ & n.d. & 2.58 & 1.84 & n.d. & n.d. & n.d. & 5.78 & 8.58 & 5.12 & n.d. & 0.52 \\
\hline 17/09/2017 & 4.20 & 2.85 & 2.25 & n.d. & n.d. & 196.17 & 6.75 & 19.36 & 6.41 & n.d. & 0.69 \\
\hline 27/09/2017 & n.d. & 2.52 & 2.24 & n.d. & n.d. & 136.40 & 5.88 & 102.31 & 6.96 & n.d. & 0.65 \\
\hline 04/10/2017 & n.d. & 2.60 & 1.44 & n.d. & n.d. & 56.01 & 3.65 & 8.47 & 7.16 & n.d. & 0.47 \\
\hline $18 / 10 / 2017$ & n.d. & 2.38 & 2.34 & 1.90 & n.d. & 83.31 & 5.12 & 15.42 & 5.08 & n.d. & 0.58 \\
\hline 01/11/2017 & n.d. & $<\mathrm{LOQ}$ & 1.88 & n.d. & n.d. & 195.36 & 4.44 & 18.97 & 2.02 & n.d. & 0.44 \\
\hline $16 / 11 / 2017$ & n.d. & 2.90 & 2.02 & n.d. & n.d. & 467.51 & 4.79 & 22.22 & 4.55 & n.d. & n.d. \\
\hline $12 / 12 / 2017$ & n.d. & 3.04 & 1.99 & n.d. & n.d. & 420.86 & 4.31 & 15.15 & 4.63 & n.d. & 0.51 \\
\hline
\end{tabular}




\begin{tabular}{|c|c|c|c|c|c|c|c|c|c|c|c|c|}
\hline $\begin{array}{l}\text { Concentra- } \\
\text { tion (ng/L) }\end{array}$ & Simazine & DCPU & Propoxur & Metribuzin & Atrazine & DCPMU & Dichlorvos & Carbofuran & Methiocarb & Ametryn & Tebuthiuron & $\begin{array}{r}\text { Pro- } \\
\text { pazine }\end{array}$ \\
\hline 29/03/2017 & 51.36 & n.d. & n.d. & n.d. & 12.91 & n.d. & n.d. & n.d. & n.d. & n.d. & $<\mathrm{LOQ}$ & n.d. \\
\hline $31 / 03 / 2017$ & 19.05 & n.d. & n.d. & n.d. & 3.65 & n.d. & n.d. & n.d. & n.d. & n.d. & $<L O Q$ & n.d. \\
\hline $01 / 04 / 2017$ & 12.76 & n.d. & n.d. & n.d. & 1.26 & n.d. & n.d. & n.d. & n.d. & n.d. & 0.55 & n.d. \\
\hline 02/04/2017 & 0.81 & n.d. & n.d. & n.d. & 4.56 & n.d. & n.d. & n.d. & n.d. & n.d. & 0.76 & n.d. \\
\hline $03 / 04 / 2017$ & 7.86 & n.d. & n.d. & n.d. & 2.85 & n.d. & n.d. & n.d. & n.d. & n.d. & 0.36 & n.d. \\
\hline 04/04/2017 & 6.08 & n.d. & n.d. & n.d. & 4.11 & n.d. & n.d. & n.d. & n.d. & n.d. & 0.77 & n.d. \\
\hline 05/04/2017 & 1.91 & n.d. & n.d. & n.d. & 4.15 & n.d. & n.d. & n.d. & n.d. & n.d. & 1.17 & n.d. \\
\hline 06/04/2017 & 1.54 & n.d. & n.d. & n.d. & 4.58 & n.d. & n.d. & n.d. & n.d. & n.d. & 1.30 & n.d. \\
\hline 07/04/2017 & 2.90 & n.d. & n.d. & n.d. & 4.21 & n.d. & n.d. & n.d. & n.d. & n.d. & 1.53 & n.d. \\
\hline 09/04/2017 & 1.69 & n.d. & n.d. & n.d. & 5.03 & n.d. & n.d. & n.d. & n.d. & n.d. & 1.69 & n.d. \\
\hline $10 / 04 / 2017$ & 1.82 & n.d. & n.d. & n.d. & 5.59 & n.d. & n.d. & n.d. & n.d. & n.d. & 1.76 & n.d. \\
\hline $12 / 04 / 2017$ & 1.41 & n.d. & n.d. & n.d. & 6.68 & n.d. & n.d. & n.d. & n.d. & n.d. & 2.06 & n.d. \\
\hline $14 / 04 / 2017$ & 2.27 & n.d. & n.d. & n.d. & 6.22 & n.d. & n.d. & n.d. & n.d. & n.d. & 1.87 & n.d. \\
\hline $15 / 04 / 2017$ & 2.50 & n.d. & n.d. & n.d. & 7.11 & n.d. & n.d. & n.d. & n.d. & n.d. & 1.81 & n.d. \\
\hline $17 / 04 / 2017$ & 2.08 & n.d. & n.d. & n.d. & 6.83 & n.d. & n.d. & n.d. & n.d. & n.d. & 1.83 & n.d. \\
\hline $19 / 04 / 2017$ & 2.92 & n.d. & n.d. & n.d. & 7.12 & n.d. & n.d. & n.d. & n.d. & n.d. & 1.86 & n.d. \\
\hline $26 / 04 / 2017$ & 2.61 & n.d. & n.d. & n.d. & 8.56 & n.d. & n.d. & n.d. & n.d. & n.d. & 1.82 & n.d. \\
\hline $03 / 05 / 2017$ & 3.16 & n.d. & n.d. & n.d. & 9.19 & n.d. & n.d. & n.d. & n.d. & n.d. & 1.81 & n.d. \\
\hline $11 / 05 / 2017$ & 2.56 & n.d. & n.d. & n.d. & 10.37 & n.d. & n.d. & n.d. & n.d. & n.d. & 1.87 & n.d. \\
\hline $17 / 05 / 2017$ & 3.08 & n.d. & n.d. & n.d. & 12.74 & n.d. & n.d. & n.d. & n.d. & n.d. & 1.64 & n.d. \\
\hline $24 / 05 / 2017$ & 6.62 & n.d. & n.d. & n.d. & 13.88 & n.d. & n.d. & n.d. & n.d. & n.d. & 1.60 & n.d. \\
\hline $31 / 05 / 2017$ & 5.17 & n.d. & n.d. & n.d. & 11.82 & n.d. & n.d. & n.d. & n.d. & n.d. & 1.37 & n.d. \\
\hline 07/06/2017 & 6.47 & n.d. & n.d. & n.d. & 13.13 & n.d. & n.d. & n.d. & n.d. & n.d. & 1.44 & n.d. \\
\hline $21 / 06 / 2017$ & 7.32 & n.d. & n.d. & n.d. & 12.11 & n.d. & n.d. & n.d. & n.d. & n.d. & 1.22 & n.d. \\
\hline 29/06/2017 & 9.22 & n.d. & n.d. & n.d. & 11.96 & n.d. & n.d. & n.d. & n.d. & n.d. & 1.28 & n.d. \\
\hline 05/07/2017 & 9.16 & n.d. & n.d. & n.d. & 13.09 & n.d. & n.d. & n.d. & n.d. & n.d. & 1.34 & n.d. \\
\hline $19 / 07 / 2017$ & 10.28 & n.d. & n.d. & n.d. & 19.55 & n.d. & n.d. & n.d. & n.d. & n.d. & 1.18 & n.d. \\
\hline $27 / 07 / 2017$ & 9.78 & n.d. & n.d. & n.d. & 19.87 & n.d. & n.d. & n.d. & n.d. & n.d. & 0.83 & n.d. \\
\hline 02/08/2017 & 10.56 & n.d. & n.d. & n.d. & 19.57 & n.d. & n.d. & n.d. & n.d. & n.d. & 0.66 & n.d. \\
\hline $30 / 08 / 2017$ & 10.49 & n.d. & n.d. & n.d. & 17.50 & n.d. & n.d. & n.d. & n.d. & n.d. & 0.87 & n.d. \\
\hline $17 / 09 / 2017$ & 15.04 & n.d. & n.d. & n.d. & 42.55 & n.d. & n.d. & n.d. & n.d. & n.d. & 1.01 & n.d. \\
\hline $27 / 09 / 2017$ & 11.42 & n.d. & n.d. & n.d. & 45.40 & n.d. & n.d. & n.d. & n.d. & n.d. & 0.88 & n.d. \\
\hline 04/10/2017 & 9.04 & n.d. & n.d. & n.d. & 34.51 & n.d. & n.d. & n.d. & n.d. & n.d. & 0.73 & n.d. \\
\hline $18 / 10 / 2017$ & 17.78 & n.d. & n.d. & n.d. & 27.25 & n.d. & n.d. & n.d. & n.d. & n.d. & 0.84 & n.d. \\
\hline $01 / 11 / 2017$ & 11.51 & n.d. & n.d. & n.d. & 12.14 & n.d. & n.d. & n.d. & n.d. & n.d. & $<\mathrm{LOQ}$ & n.d. \\
\hline $16 / 11 / 2017$ & 10.51 & n.d. & n.d. & n.d. & 12.19 & n.d. & n.d. & n.d. & n.d. & n.d. & 0.62 & n.d. \\
\hline $12 / 12 / 2017$ & 26.67 & n.d. & n.d. & n.d. & 11.38 & n.d. & n.d. & n.d. & n.d. & n.d. & 0.48 & n.d. \\
\hline
\end{tabular}




\begin{tabular}{|c|c|c|c|c|c|c|c|c|c|c|c|}
\hline $\begin{array}{l}\text { Concentra- } \\
\text { tion (ng/L) }\end{array}$ & Terbuthylazine & Asulam & Naproxen & Diuron & Fluometuron & Carbamazepine & Prometryn & Terbutryn & Picloram & Hexazinone & Fluroxypyr \\
\hline 29/03/2017 & 5.71 & n.d. & n.d. & 7.62 & 0.94 & 70.02 & 0.95 & n.d. & n.d. & 3.63 & 3.77 \\
\hline $31 / 03 / 2017$ & n.d. & n.d. & n.d. & 5.99 & n.d. & 1.67 & n.d. & n.d. & n.d. & n.d. & 5.53 \\
\hline $01 / 04 / 2017$ & n.d. & n.d. & n.d. & 4.31 & n.d. & 2.01 & 1.75 & n.d. & n.d. & n.d. & 5.26 \\
\hline 02/04/2017 & n.d. & n.d. & n.d. & 0.84 & n.d. & n.d. & 2.10 & n.d. & n.d. & $<\mathrm{LOQ}$ & n.d. \\
\hline 03/04/2017 & n.d. & n.d. & n.d. & 1.80 & n.d. & 2.48 & 1.79 & n.d. & n.d. & n.d. & n.d. \\
\hline 04/04/2017 & n.d. & n.d. & n.d. & 1.28 & n.d. & 2.91 & 2.40 & n.d. & n.d. & n.d. & n.d. \\
\hline 05/04/2017 & n.d. & n.d. & n.d. & 1.64 & n.d. & 3.15 & 2.51 & n.d. & n.d. & $<\mathrm{LOQ}$ & n.d. \\
\hline 06/04/2017 & n.d. & n.d. & n.d. & 1.27 & n.d. & 3.90 & 2.41 & n.d. & n.d. & $<\mathrm{LOQ}$ & n.d. \\
\hline 07/04/2017 & n.d. & n.d. & n.d. & 0.91 & n.d. & 3.11 & 2.07 & n.d. & n.d. & $<\mathrm{LOQ}$ & n.d. \\
\hline 09/04/2017 & n.d. & n.d. & n.d. & 1.24 & n.d. & 6.62 & 1.93 & n.d. & n.d. & $<\mathrm{LOQ}$ & n.d. \\
\hline $10 / 04 / 2017$ & n.d. & n.d. & n.d. & 1.52 & $<L O Q$ & 7.02 & 1.98 & n.d. & n.d. & $<L O Q$ & n.d. \\
\hline $12 / 04 / 2017$ & n.d. & n.d. & n.d. & 1.37 & $<\mathrm{LOQ}$ & 8.92 & 1.76 & n.d. & n.d. & $<\mathrm{LOQ}$ & n.d. \\
\hline $14 / 04 / 2017$ & n.d. & n.d. & n.d. & 1.61 & $<\mathrm{LOQ}$ & 7.92 & 1.74 & n.d. & n.d. & $<\mathrm{LOQ}$ & n.d. \\
\hline $15 / 04 / 2017$ & n.d. & n.d. & n.d. & 1.76 & $<\mathrm{LOQ}$ & 9.70 & 1.65 & n.d. & n.d. & $<L O Q$ & n.d. \\
\hline $17 / 04 / 2017$ & n.d. & n.d. & n.d. & 2.31 & $<\mathrm{LOQ}$ & 8.36 & 1.59 & n.d. & n.d. & $<L O Q$ & n.d. \\
\hline $19 / 04 / 2017$ & n.d. & n.d. & n.d. & 2.15 & $<\mathrm{LOQ}$ & 12.95 & 1.54 & n.d. & n.d. & $<L O Q$ & n.d. \\
\hline $26 / 04 / 2017$ & n.d. & n.d. & n.d. & 2.73 & $<\mathrm{LOQ}$ & 15.92 & 1.67 & n.d. & n.d. & 1.08 & n.d. \\
\hline $03 / 05 / 2017$ & n.d. & n.d. & n.d. & 3.41 & 0.30 & 21.73 & 1.45 & n.d. & n.d. & 1.04 & n.d. \\
\hline $11 / 05 / 2017$ & n.d. & n.d. & n.d. & 4.08 & 0.32 & 20.62 & 1.49 & n.d. & n.d. & 1.30 & n.d. \\
\hline $17 / 05 / 2017$ & n.d. & n.d. & n.d. & 5.26 & 0.35 & 41.29 & 1.41 & n.d. & n.d. & 1.12 & n.d. \\
\hline $24 / 05 / 2017$ & n.d. & n.d. & n.d. & 6.94 & $<\mathrm{LOQ}$ & 46.37 & 1.43 & n.d. & n.d. & 1.22 & n.d. \\
\hline $31 / 05 / 2017$ & n.d. & n.d. & n.d. & 6.06 & 0.31 & 39.21 & 1.23 & n.d. & n.d. & 0.99 & n.d. \\
\hline 07/06/2017 & n.d. & n.d. & n.d. & 8.17 & 0.33 & 43.82 & 1.32 & n.d. & n.d. & 1.19 & n.d. \\
\hline $21 / 06 / 2017$ & 3.04 & n.d. & n.d. & 9.96 & 0.32 & 45.83 & 1.18 & n.d. & n.d. & 1.10 & n.d. \\
\hline 29/06/2017 & n.d. & n.d. & n.d. & 9.75 & 0.30 & 66.16 & 1.17 & n.d. & n.d. & 1.20 & n.d. \\
\hline 05/07/2017 & 7.72 & n.d. & n.d. & 10.88 & 0.37 & 50.85 & 1.24 & n.d. & n.d. & 1.23 & n.d. \\
\hline $19 / 07 / 2017$ & 12.71 & n.d. & n.d. & 11.46 & 0.34 & 57.92 & 1.06 & n.d. & n.d. & 1.70 & n.d. \\
\hline 27/07/2017 & 39.90 & n.d. & n.d. & 11.89 & $<\mathrm{LOQ}$ & 56.95 & 1.11 & n.d. & n.d. & 1.84 & n.d. \\
\hline $02 / 08 / 2017$ & 38.89 & n.d. & n.d. & 11.03 & $<\mathrm{LOQ}$ & 85.65 & 1.36 & n.d. & n.d. & 2.60 & n.d. \\
\hline $30 / 08 / 2017$ & 69.14 & n.d. & n.d. & 11.79 & $<\mathrm{LOQ}$ & 84.95 & 0.82 & n.d. & n.d. & 2.59 & n.d. \\
\hline $17 / 09 / 2017$ & 98.14 & n.d. & n.d. & 26.30 & 0.69 & 71.39 & 1.18 & n.d. & n.d. & 3.46 & 4.56 \\
\hline 27/09/2017 & 83.06 & n.d. & n.d. & 22.09 & 1.00 & 89.07 & 1.15 & n.d. & n.d. & 2.93 & 12.31 \\
\hline $04 / 10 / 2017$ & 76.57 & n.d. & n.d. & 17.53 & 0.88 & 69.41 & 1.06 & n.d. & n.d. & 2.15 & 9.38 \\
\hline $18 / 10 / 2017$ & 59.04 & n.d. & n.d. & 20.27 & 0.51 & 78.21 & 0.67 & n.d. & n.d. & 3.85 & 6.23 \\
\hline $01 / 11 / 2017$ & 22.87 & n.d. & n.d. & 9.59 & n.d. & 94.63 & n.d. & n.d. & n.d. & 4.28 & 1.86 \\
\hline $16 / 11 / 2017$ & 14.55 & n.d. & n.d. & 10.94 & $<\mathrm{LOQ}$ & 73.66 & n.d. & n.d. & n.d. & 4.97 & n.d. \\
\hline $12 / 12 / 2017$ & 8.33 & n.d. & n.d. & 10.74 & n.d. & 63.29 & $<\mathrm{LOQ}$ & n.d. & n.d. & 4.35 & n.d. \\
\hline
\end{tabular}




\begin{tabular}{|c|c|c|c|c|c|c|c|c|c|c|c|}
\hline $\begin{array}{l}\text { Concentra- } \\
\text { tion (ng/L) }\end{array}$ & Imidacloprid & Bromacil & Tramadol & Atenolol & $\begin{array}{r}\text { Desmethyl- } \\
\text { Diazepam }\end{array}$ & Imazapic & Venlafaxine & Metalaxyl & Pendimethalin & Metolachlor & Imazethapyr \\
\hline 29/03/2017 & 15.86 & n.d. & 28.64 & 2.20 & 0.91 & n.d. & 11.23 & $<\mathrm{LOQ}$ & n.d. & 44.88 & 0.17 \\
\hline $31 / 03 / 2017$ & 9.38 & n.d. & 0.98 & 0.98 & n.d. & 0.73 & 0.45 & n.d. & n.d. & 8.95 & n.d. \\
\hline 01/04/2017 & 13.02 & n.d. & 1.13 & n.d. & n.d. & n.d. & 0.16 & 4.32 & n.d. & 16.07 & n.d. \\
\hline 02/04/2017 & 21.47 & n.d. & 0.83 & 0.35 & n.d. & n.d. & 0.53 & 0.23 & n.d. & 141.62 & 0.47 \\
\hline 03/04/2017 & 15.18 & n.d. & 2.30 & 0.38 & $<\mathrm{LOQ}$ & n.d. & 0.83 & 0.57 & n.d. & 75.22 & 0.31 \\
\hline 04/04/2017 & 16.93 & n.d. & 3.09 & 0.54 & n.d. & n.d. & 1.62 & 0.76 & n.d. & 259.49 & 0.41 \\
\hline 05/04/2017 & 18.13 & n.d. & 2.92 & 0.61 & n.d. & n.d. & 1.86 & 0.84 & n.d. & 115.44 & 0.56 \\
\hline 06/04/2017 & 17.76 & n.d. & 3.75 & 1.06 & $<L O Q$ & n.d. & 2.49 & 0.82 & n.d. & 96.07 & 0.65 \\
\hline 07/04/2017 & 15.26 & n.d. & 3.84 & 0.70 & $<\mathrm{LOQ}$ & n.d. & 2.49 & 0.74 & n.d. & 84.97 & 0.60 \\
\hline 09/04/2017 & 16.18 & n.d. & 5.66 & 1.05 & $<\mathrm{LOQ}$ & n.d. & 3.60 & 0.60 & n.d. & 234.17 & 0.49 \\
\hline 10/04/2017 & 17.59 & n.d. & 6.42 & 1.23 & $<\mathrm{LOQ}$ & n.d. & 4.79 & 0.54 & n.d. & 259.37 & 0.60 \\
\hline $12 / 04 / 2017$ & 15.88 & n.d. & 6.74 & 1.32 & $<\mathrm{LOQ}$ & n.d. & 4.89 & 0.57 & n.d. & 85.86 & 0.50 \\
\hline $14 / 04 / 2017$ & 17.34 & n.d. & 9.27 & 3.89 & 0.24 & n.d. & 6.29 & 0.57 & n.d. & 78.27 & 0.43 \\
\hline $15 / 04 / 2017$ & 16.18 & n.d. & 8.38 & 1.50 & $<\mathrm{LOQ}$ & n.d. & 6.48 & 0.52 & n.d. & 77.83 & 0.40 \\
\hline 17/04/2017 & 14.84 & n.d. & 9.40 & 1.82 & 0.25 & n.d. & 6.09 & 0.57 & n.d. & 75.71 & 0.44 \\
\hline 19/04/2017 & 16.37 & n.d. & 11.41 & 1.79 & 0.29 & n.d. & 9.49 & 0.50 & n.d. & 75.23 & 0.52 \\
\hline 26/04/2017 & 16.79 & n.d. & 16.56 & 2.33 & 0.32 & n.d. & 11.75 & 0.60 & n.d. & 84.98 & 0.57 \\
\hline 03/05/2017 & 16.02 & n.d. & 18.88 & 13.11 & 0.55 & n.d. & 11.08 & 0.56 & n.d. & 75.27 & 0.49 \\
\hline $11 / 05 / 2017$ & 16.32 & n.d. & 23.87 & 10.46 & 0.72 & n.d. & 15.91 & 0.64 & n.d. & 73.04 & 0.64 \\
\hline $17 / 05 / 2017$ & 16.49 & n.d. & 26.40 & 8.14 & 0.72 & n.d. & 16.83 & 0.57 & n.d. & 102.49 & 0.48 \\
\hline 24/05/2017 & 17.17 & n.d. & 34.61 & 25.11 & 0.86 & n.d. & 21.54 & 0.59 & n.d. & 103.57 & 0.52 \\
\hline $31 / 05 / 2017$ & 15.61 & n.d. & 32.02 & 13.46 & 0.89 & n.d. & 19.90 & 0.54 & n.d. & 54.48 & 0.42 \\
\hline 07/06/2017 & 17.31 & n.d. & 42.26 & 12.95 & 1.01 & n.d. & 25.67 & 0.55 & n.d. & 55.71 & 0.50 \\
\hline $21 / 06 / 2017$ & 17.15 & n.d. & 46.03 & 14.22 & 1.06 & n.d. & 32.37 & 0.55 & n.d. & 87.58 & 0.47 \\
\hline 29/06/2017 & 16.19 & n.d. & 50.75 & 15.11 & 1.39 & n.d. & 33.70 & 0.52 & n.d. & 92.02 & 0.41 \\
\hline 05/07/2017 & 16.77 & n.d. & 63.52 & 14.42 & 1.59 & n.d. & 37.63 & 0.61 & n.d. & 79.16 & 0.47 \\
\hline 19/07/2017 & 22.99 & n.d. & 68.15 & 13.79 & 1.52 & n.d. & 41.33 & 0.57 & n.d. & 53.81 & 0.77 \\
\hline $27 / 07 / 2017$ & 19.23 & n.d. & 61.13 & 10.95 & 1.22 & 0.68 & 41.10 & 0.44 & n.d. & 82.14 & 0.79 \\
\hline 02/08/2017 & 21.02 & n.d. & 74.46 & 12.90 & 1.05 & n.d. & 43.57 & 0.32 & n.d. & 94.82 & 0.47 \\
\hline $30 / 08 / 2017$ & 17.82 & n.d. & 49.96 & 7.47 & 1.62 & n.d. & 28.47 & 0.45 & n.d. & 47.97 & 0.59 \\
\hline 17/09/2017 & 133.18 & n.d. & 63.98 & 12.30 & 1.90 & 2.34 & 42.35 & 0.76 & n.d. & 96.50 & 0.69 \\
\hline 27/09/2017 & 261.85 & n.d. & 48.74 & 10.39 & 1.97 & 3.17 & 41.09 & 0.92 & n.d. & 143.16 & 0.28 \\
\hline 04/10/2017 & 157.40 & n.d. & 42.69 & 8.00 & 2.20 & 3.19 & 38.79 & 0.78 & n.d. & 125.81 & 0.37 \\
\hline 18/10/2017 & 178.19 & n.d. & 55.84 & 8.93 & 1.82 & 2.54 & 40.11 & 0.58 & n.d. & 53.90 & 0.40 \\
\hline 01/11/2017 & 67.05 & n.d. & 38.45 & 10.24 & 0.73 & n.d. & 27.44 & n.d. & n.d. & 43.59 & n.d. \\
\hline $16 / 11 / 2017$ & 48.77 & n.d. & 41.84 & 7.70 & 1.46 & n.d. & 29.76 & 0.35 & n.d. & 38.08 & 0.16 \\
\hline $12 / 12 / 2017$ & 21.82 & n.d. & 31.84 & 3.71 & 1.18 & n.d. & 23.31 & 0.27 & n.d. & 37.68 & 0.31 \\
\hline
\end{tabular}




\begin{tabular}{|c|c|c|c|c|c|c|c|c|c|c|c|}
\hline $\begin{array}{l}\text { Concentra- } \\
\text { tion }(\mathrm{ng} / \mathrm{L})\end{array}$ & Codeine & Temazepam & Fenamiphos & Diazinon & Tebuconazole & Fluoxetine & $\begin{array}{l}\text { Desmethyl } \\
\text { Citalopram }\end{array}$ & Citalopram & Fluazifop & Malathion & Propiconazole \\
\hline 29/03/2017 & n.d. & 23.24 & n.d. & 0.82 & n.d. & n.d. & n.d. & n.d. & n.d. & n.d. & n.d. \\
\hline $31 / 03 / 2017$ & 0.79 & 0.62 & n.d. & n.d. & n.d. & n.d. & n.d. & n.d. & n.d. & n.d. & n.d. \\
\hline 01/04/2017 & n.d. & 0.91 & n.d. & n.d. & n.d. & n.d. & n.d. & n.d. & n.d. & n.d. & n.d. \\
\hline 02/04/2017 & n.d. & n.d. & n.d. & 0.14 & n.d. & n.d. & $<L O Q$ & n.d. & 8.95 & n.d. & n.d. \\
\hline 03/04/2017 & n.d. & 1.10 & n.d. & 0.30 & n.d. & n.d. & n.d. & n.d. & 2.43 & n.d. & n.d. \\
\hline 04/04/2017 & n.d. & 1.12 & n.d. & 0.40 & n.d. & n.d. & n.d. & n.d. & 1.55 & n.d. & n.d. \\
\hline 05/04/2017 & 0.54 & 1.02 & n.d. & 0.61 & n.d. & n.d. & n.d. & n.d. & 1.25 & n.d. & n.d. \\
\hline 06/04/2017 & 0.67 & 1.17 & n.d. & 0.75 & n.d. & n.d. & n.d. & n.d. & n.d. & n.d. & n.d. \\
\hline 07/04/2017 & 0.43 & 1.32 & n.d. & 0.72 & n.d. & n.d. & n.d. & n.d. & n.d. & n.d. & n.d. \\
\hline 09/04/2017 & 0.57 & 2.09 & n.d. & 0.86 & n.d. & n.d. & n.d. & n.d. & n.d. & n.d. & n.d. \\
\hline $10 / 04 / 2017$ & n.d. & 2.41 & n.d. & 1.01 & n.d. & n.d. & n.d. & n.d. & n.d. & n.d. & n.d. \\
\hline $12 / 04 / 2017$ & 0.58 & 2.74 & n.d. & 1.08 & n.d. & n.d. & n.d. & n.d. & n.d. & n.d. & n.d. \\
\hline $14 / 04 / 2017$ & 1.38 & 3.22 & n.d. & 0.95 & n.d. & n.d. & n.d. & n.d. & n.d. & n.d. & n.d. \\
\hline $15 / 04 / 2017$ & 0.93 & 3.54 & n.d. & 0.93 & n.d. & n.d. & n.d. & n.d. & n.d. & n.d. & n.d. \\
\hline $17 / 04 / 2017$ & 0.67 & 4.10 & n.d. & 0.92 & n.d. & n.d. & n.d. & n.d. & n.d. & n.d. & n.d. \\
\hline $19 / 04 / 2017$ & 0.71 & 4.44 & n.d. & 0.83 & n.d. & n.d. & n.d. & n.d. & n.d. & n.d. & n.d. \\
\hline $26 / 04 / 2017$ & 0.62 & 6.21 & n.d. & 0.89 & n.d. & n.d. & n.d. & n.d. & n.d. & n.d. & n.d. \\
\hline 03/05/2017 & 0.77 & 7.81 & n.d. & 0.89 & n.d. & n.d. & n.d. & n.d. & n.d. & n.d. & n.d. \\
\hline $11 / 05 / 2017$ & 0.80 & 8.57 & n.d. & 0.76 & n.d. & n.d. & n.d. & n.d. & n.d. & n.d. & n.d. \\
\hline $17 / 05 / 2017$ & 0.80 & 10.37 & n.d. & 0.84 & n.d. & n.d. & n.d. & n.d. & n.d. & n.d. & n.d. \\
\hline $24 / 05 / 2017$ & 1.17 & 12.16 & n.d. & 1.02 & n.d. & n.d. & n.d. & n.d. & n.d. & n.d. & n.d. \\
\hline $31 / 05 / 2017$ & 1.14 & 12.33 & n.d. & 0.67 & n.d. & n.d. & n.d. & n.d. & n.d. & n.d. & n.d. \\
\hline 07/06/2017 & 1.62 & 13.54 & n.d. & 0.75 & n.d. & n.d. & n.d. & 1.99 & n.d. & n.d. & n.d. \\
\hline 21/06/2017 & 2.49 & 15.56 & n.d. & 0.67 & n.d. & n.d. & n.d. & n.d. & n.d. & n.d. & n.d. \\
\hline 29/06/2017 & 3.01 & 18.14 & n.d. & 0.74 & n.d. & n.d. & n.d. & 2.40 & n.d. & n.d. & n.d. \\
\hline 05/07/2017 & 3.90 & 19.89 & n.d. & 0.78 & n.d. & n.d. & n.d. & n.d. & n.d. & n.d. & n.d. \\
\hline 19/07/2017 & 4.86 & 22.16 & n.d. & 0.76 & n.d. & n.d. & n.d. & n.d. & n.d. & n.d. & n.d. \\
\hline $27 / 07 / 2017$ & 4.23 & 23.19 & n.d. & 0.79 & n.d. & n.d. & n.d. & n.d. & n.d. & n.d. & n.d. \\
\hline 02/08/2017 & 5.34 & 23.18 & n.d. & 0.92 & n.d. & n.d. & n.d. & n.d. & n.d. & n.d. & n.d. \\
\hline $30 / 08 / 2017$ & n.d. & 24.34 & n.d. & 0.40 & n.d. & n.d. & n.d. & n.d. & n.d. & n.d. & n.d. \\
\hline $17 / 09 / 2017$ & 1.33 & 27.15 & n.d. & 0.71 & n.d. & n.d. & n.d. & n.d. & n.d. & n.d. & n.d. \\
\hline 27/09/2017 & 0.71 & 28.11 & n.d. & 0.69 & n.d. & n.d. & n.d. & 3.14 & n.d. & n.d. & n.d. \\
\hline 04/10/2017 & 0.57 & 23.73 & n.d. & 0.60 & n.d. & n.d. & n.d. & 3.64 & n.d. & n.d. & n.d. \\
\hline $18 / 10 / 2017$ & 1.34 & 26.89 & n.d. & 0.53 & n.d. & n.d. & n.d. & n.d. & n.d. & n.d. & n.d. \\
\hline $01 / 11 / 2017$ & 0.87 & 20.43 & n.d. & n.d. & n.d. & n.d. & n.d. & n.d. & n.d. & n.d. & n.d. \\
\hline $16 / 11 / 2017$ & 1.83 & 19.50 & n.d. & 0.29 & n.d. & n.d. & n.d. & n.d. & n.d. & n.d. & n.d. \\
\hline $12 / 12 / 2017$ & 0.88 & 14.96 & n.d. & 0.26 & n.d. & n.d. & n.d. & n.d. & n.d. & n.d. & n.d. \\
\hline
\end{tabular}




\begin{tabular}{|c|c|c|c|c|c|c|c|c|c|}
\hline $\begin{array}{l}\text { Concentra- } \\
\text { tion (ng/L) }\end{array}$ & Prothioconazole & Chlorpyriphos & Haloxyfop + & $\begin{array}{r}\text { Metsulfuron- } \\
\text { Methyl } \\
\end{array}$ & Tadalafil & Verapamil & Sildenafil & Atorvastatin & Iopromide \\
\hline 29/03/2017 & n.d. & n.d. & n.d. & n.d. & n.d. & n.d. & n.d. & n.d. & 432.30 \\
\hline $31 / 03 / 2017$ & n.d. & n.d. & n.d. & n.d. & n.d. & n.d. & n.d. & n.d. & 28.65 \\
\hline 01/04/2017 & n.d. & n.d. & n.d. & n.d. & n.d. & n.d. & n.d. & n.d. & 6.99 \\
\hline 02/04/2017 & n.d. & n.d. & n.d. & n.d. & n.d. & n.d. & n.d. & n.d. & 20.48 \\
\hline 03/04/2017 & n.d. & n.d. & n.d. & n.d. & n.d. & n.d. & n.d. & n.d. & 26.37 \\
\hline 04/04/2017 & n.d. & n.d. & n.d. & n.d. & n.d. & n.d. & n.d. & n.d. & 50.65 \\
\hline 05/04/2017 & n.d. & n.d. & n.d. & n.d. & n.d. & n.d. & n.d. & n.d. & 61.06 \\
\hline 06/04/2017 & n.d. & n.d. & n.d. & n.d. & n.d. & n.d. & n.d. & n.d. & 66.40 \\
\hline 07/04/2017 & n.d. & n.d. & n.d. & n.d. & n.d. & n.d. & n.d. & n.d. & 73.22 \\
\hline 09/04/2017 & n.d. & n.d. & n.d. & n.d. & n.d. & n.d. & n.d. & n.d. & 113.38 \\
\hline 10/04/2017 & n.d. & n.d. & n.d. & n.d. & n.d. & n.d. & n.d. & n.d. & 130.25 \\
\hline $12 / 04 / 2017$ & n.d. & n.d. & n.d. & n.d. & n.d. & n.d. & n.d. & n.d. & 185.32 \\
\hline 14/04/2017 & n.d. & n.d. & n.d. & n.d. & n.d. & n.d. & n.d. & n.d. & 160.55 \\
\hline $15 / 04 / 2017$ & n.d. & n.d. & n.d. & n.d. & n.d. & n.d. & n.d. & n.d. & 189.39 \\
\hline $17 / 04 / 2017$ & n.d. & n.d. & n.d. & n.d. & n.d. & n.d. & n.d. & n.d. & 182.18 \\
\hline 19/04/2017 & n.d. & n.d. & n.d. & n.d. & n.d. & n.d. & n.d. & n.d. & 180.08 \\
\hline 26/04/2017 & n.d. & n.d. & n.d. & n.d. & n.d. & n.d. & n.d. & n.d. & 206.21 \\
\hline 03/05/2017 & n.d. & n.d. & n.d. & n.d. & n.d. & n.d. & n.d. & n.d. & 258.99 \\
\hline $11 / 05 / 2017$ & n.d. & n.d. & n.d. & n.d. & n.d. & n.d. & n.d. & n.d. & 266.10 \\
\hline $17 / 05 / 2017$ & n.d. & n.d. & n.d. & n.d. & n.d. & n.d. & n.d. & n.d. & 334.14 \\
\hline 24/05/2017 & n.d. & n.d. & n.d. & n.d. & n.d. & n.d. & n.d. & n.d. & 333.45 \\
\hline $31 / 05 / 2017$ & n.d. & n.d. & n.d. & n.d. & n.d. & n.d. & n.d. & n.d. & 401.00 \\
\hline 07/06/2017 & n.d. & n.d. & n.d. & n.d. & n.d. & n.d. & n.d. & n.d. & 364.17 \\
\hline 21/06/2017 & n.d. & n.d. & n.d. & n.d. & n.d. & n.d. & n.d. & n.d. & 311.15 \\
\hline 29/06/2017 & n.d. & n.d. & n.d. & n.d. & n.d. & n.d. & n.d. & n.d. & 437.19 \\
\hline 05/07/2017 & n.d. & n.d. & n.d. & n.d. & n.d. & n.d. & n.d. & n.d. & 442.72 \\
\hline 19/07/2017 & n.d. & n.d. & n.d. & n.d. & n.d. & n.d. & n.d. & n.d. & 645.33 \\
\hline 27/07/2017 & n.d. & n.d. & n.d. & n.d. & n.d. & n.d. & n.d. & n.d. & 515.36 \\
\hline 02/08/2017 & n.d. & n.d. & n.d. & n.d. & n.d. & n.d. & n.d. & n.d. & 750.17 \\
\hline $30 / 08 / 2017$ & n.d. & n.d. & n.d. & n.d. & n.d. & n.d. & n.d. & n.d. & 669.31 \\
\hline 17/09/2017 & n.d. & n.d. & n.d. & n.d. & n.d. & n.d. & n.d. & n.d. & 591.67 \\
\hline 27/09/2017 & n.d. & n.d. & n.d. & n.d. & n.d. & n.d. & n.d. & n.d. & 432.73 \\
\hline 04/10/2017 & n.d. & n.d. & n.d. & n.d. & n.d. & n.d. & 0.45 & n.d. & 289.05 \\
\hline 18/10/2017 & n.d. & n.d. & n.d. & n.d. & n.d. & n.d. & n.d. & n.d. & 570.55 \\
\hline 01/11/2017 & n.d. & n.d. & n.d. & n.d. & n.d. & n.d. & n.d. & n.d. & 450.16 \\
\hline $16 / 11 / 2017$ & n.d. & n.d. & n.d. & n.d. & n.d. & n.d. & n.d. & n.d. & 364.64 \\
\hline $12 / 12 / 2017$ & n.d. & n.d. & n.d. & n.d. & n.d. & n.d. & n.d. & n.d. & 357.21 \\
\hline
\end{tabular}


Table S-12. Rate constant for chemical loss from the estuary segment and emission flux estimated by fitting the measured concentrations in river water to Eq. 2 (time invariant rate constant) for Type B compounds.

\begin{tabular}{|c|c|c|c|c|c|c|c|c|c|}
\hline & \multicolumn{3}{|c|}{$k_{r}(1 /$ day $)$} & \multicolumn{2}{|c|}{ Half-life (days) } & \multicolumn{4}{|c|}{ Chemical input $\mathrm{E}$ (mg/day) } \\
\hline & Median & $95 \% \mathrm{CI}$ & $\mathrm{R}^{2}$ & Median & $95 \% \mathrm{CI}$ & Modelled (Median) & Modelled (95\% CI) & $\begin{array}{c}\text { Measured at } \\
\text { WWTP }\end{array}$ & Meas/Mod \\
\hline Carbamazepine & $1.0 \cdot 10^{-2}$ & $7.4 \cdot 10^{-3}$ to $1.4 \cdot 10^{-2}$ & 0.94 & 69 & 51 to 97 & 4000 & 3300 to 4800 & 4100 & 1.03 \\
\hline Cotinine & $5.9 \cdot 10^{-3}$ & $2.3 \cdot 10^{-3}$ to $1.0 \cdot 10^{-2}$ & 0.88 & 118 & 68 to 300 & 290 & 210 to 390 & 78 & 0.27 \\
\hline Desmethyl diazepam & $1.1 \cdot 10^{-2}$ & $6.7 \cdot 10^{-3}$ to $1.7 \cdot 10^{-2}$ & 0.89 & 62 & 41 to 100 & 92 & 69 to 120 & 87 & 0.95 \\
\hline Diuron & $8.0 \cdot 10^{-3}$ & $4.7 \cdot 10^{-3}$ to $1.2 \cdot 10^{-2}$ & 0.97 & 86 & 59 to 150 & 610 & 510 to 720 & 620 & 1.02 \\
\hline Gabapentin & $1.8 \cdot 10^{-2}$ & $1.2 \cdot 10^{-2}$ to $2.5 \cdot 10^{-2}$ & 0.88 & 40 & 27 to 58 & 3500 & 2600 to 4700 & 920000 & 260 \\
\hline Hydrochlorothiazide & $5.8 \cdot 10^{-2}$ & $3.1 \cdot 10^{-2}$ to $1.3 \cdot 10^{-1}$ & 0.51 & 12 & 5.6 to 22 & 3800 & 2100 to 7800 & 5100 & 1.34 \\
\hline Hydroxycotinine & $1.5 \cdot 10^{-2}$ & $9.8 \cdot 10^{-3}$ to $2.2 \cdot 10^{-2}$ & 0.87 & 46 & 31 to 71 & 350 & 260 to 490 & 460 & 1.31 \\
\hline Iopromide & $2.3 \cdot 10^{-2}$ & $1.4 \cdot 10^{-2}$ to $3.9 \cdot 10^{-2}$ & 0.74 & 30 & 18 to 48 & 50000 & 34000 to 77000 & 51000 & 1.02 \\
\hline Temazepam & $1.4 \cdot 10^{-2}$ & $1.0 \cdot 10^{-2}$ to $1.8 \cdot 10^{-2}$ & 0.94 & 51 & 40 to 67 & 1500 & 1200 to 1800 & 83 & 0.55 \\
\hline Tramadol & $1.7 \cdot 10^{-2}$ & $1.1 \cdot 10^{-2}$ to $2.5 \cdot 10^{-2}$ & 0.83 & 40 & 27 to 62 & 4000 & 3000 to 5500 & - & - \\
\hline Venlafaxine & $1.8 \cdot 10^{-2}$ & $1.3 \cdot 10^{-2}$ to $2.5 \cdot 10^{-2}$ & 0.89 & 38 & 27 to 52 & 2900 & 2300 to 3800 & 9200 & 3.2 \\
\hline
\end{tabular}

Table S-13. Rate constant for chemical loss from the estuary segment and emission flux estimated by fitting the measured concentrations in river water to Eq. 5 (UV-radiation intensity dependant rate constant) for Type B compounds. Presented half-lives are based on the mean of the daily UV indices when water samples were taken.

\begin{tabular}{|c|c|c|c|c|c|c|c|c|c|}
\hline & \multicolumn{3}{|c|}{ krp (1/day) } & \multicolumn{2}{|c|}{ Half-life (days) } & \multicolumn{4}{|c|}{ Chemical input $E$ (mg/day) } \\
\hline & Median & $95 \% \mathrm{CI}$ & $\mathrm{R}^{2}$ & Median & $95 \% \mathrm{CI}$ & Modelled (Median) & Modelled (95\% CI) & $\begin{array}{c}\text { Measured at } \\
\text { WWTP }\end{array}$ & Meas/Mod \\
\hline Carbamazepine & $4.6 \cdot 10^{-3}$ & $3.2 \cdot 10^{-3}$ to $6.0 \cdot 10^{-3}$ & 0.94 & 150 & 110 to 210 & 3000 & 2600 to 3400 & 4100 & 1.37 \\
\hline Cotinine & $3.3 \cdot 10^{-3}$ & $1.8 \cdot 10^{-3}$ to $5.0 \cdot 10^{-3}$ & 0.91 & 210 & 140 to 380 & 250 & 210 to 300 & 78 & 0.31 \\
\hline Desmethyl diazepam & $4.9 \cdot 10^{-3}$ & $3.3 \cdot 10^{-3}$ to $6.7 \cdot 10^{-3}$ & 0.91 & 140 & 100 to 210 & 66 & 56 to 77 & 87 & 1.32 \\
\hline Diuron & $1.1 \cdot 10^{-2}$ & $7.1 \cdot 10^{-3}$ to $1.4 \cdot 10^{-2}$ & 0.98 & 66 & 48 to 98 & 640 & 550 to 730 & 620 & 0.97 \\
\hline Gabapentin & $7.2 \cdot 10^{-3}$ & $4.8 \cdot 10^{-3}$ to $1.0 \cdot 10^{-2}$ & 0.85 & 96 & 68 to 150 & 2100 & 1700 to 2700 & 920000 & 440 \\
\hline Hydrochlorothiazide & $3.9 \cdot 10^{-2}$ & $2.8 \cdot 10^{-2}$ to $5.9 \cdot 10^{-2}$ & 0.85 & 18 & 12 to 25 & 2900 & 2200 to 4100 & 5100 & 1.76 \\
\hline Hydroxycotinine & $6.7 \cdot 10^{-3}$ & $4.7 \cdot 10^{-3}$ to $9.1 \cdot 10^{-3}$ & 0.88 & 100 & 76 to 150 & 230 & 190 to 280 & 460 & 2.0 \\
\hline Iopromide & $1.1 \cdot 10^{-2}$ & $8.0 \cdot 10^{-3}$ to $1.6 \cdot 10^{-2}$ & 0.80 & 61 & 44 to 87 & 32000 & 26000 to 41000 & 51000 & 1.59 \\
\hline Temazepam & $7.0 \cdot 10^{-3}$ & $6.2 \cdot 10^{-3}$ to $7.8 \cdot 10^{-3}$ & 0.98 & 99 & 89 to 110 & 1100 & 1000 to 1200 & 83 & 0.75 \\
\hline Tramadol & $1.0 \cdot 10^{-2}$ & $8.1 \cdot 10^{-3}$ to $1.2 \cdot 10^{-2}$ & 0.93 & 69 & 56 to 85 & 3200 & 2800 to 3600 & - & - \\
\hline Venlafaxine & $9.6 \cdot 10^{-3}$ & $7.8 \cdot 10^{-3}$ to $1.2 \cdot 10^{-2}$ & 0.94 & 72 & 59 to 88 & 2100 & 1900 to 2400 & 9200 & 4.4 \\
\hline
\end{tabular}


Table S-14. Rate constant for chemical loss from the estuary segment and emission flux estimated by fitting the measured concentrations in river water to Eq. 7 (temperature-dependant rate constant) for Type B compounds. Presented half-lives are based on the mean of the daily water temperatures when water samples were taken.

\begin{tabular}{|c|c|c|c|c|c|c|c|c|c|}
\hline & \multicolumn{3}{|c|}{$k_{\text {rT }}$ (1/day) } & \multicolumn{2}{|c|}{ Half-life (days) } & \multicolumn{4}{|c|}{ Chemical input E (mg/day) } \\
\hline & Median & $95 \% \mathrm{CI}$ & $\mathrm{R}^{2}$ & Median & $95 \% \mathrm{CI}$ & Modelled (Median) & Modelled (95\% CI) & $\begin{array}{l}\text { Measured at } \\
\text { WWTP }\end{array}$ & Meas/Mod \\
\hline Carbamazepine & $1.1 \cdot 10^{-2}$ & $7.5 \cdot 10^{-3}$ to $1.5 \cdot 10^{-2}$ & 0.95 & 64 & 47 to 92 & 3800 & 3100 to 4700 & 4100 & 1.08 \\
\hline Cotinine & $9.0 \cdot 10^{-3}$ & $5.0 \cdot 10^{-3}$ to $1.4 \cdot 10^{-2}$ & 0.92 & 77 & 51 to 140 & 250 & 190 to 330 & 78 & 0.31 \\
\hline Desmethyl diazepam & $1.3 \cdot 10^{-2}$ & $8.7 \cdot 10^{-3}$ to $1.8 \cdot 10^{-2}$ & 0.92 & 54 & 39 to 80 & 75 & 53 to 100 & 87 & 1.16 \\
\hline Diuron & $7.9 \cdot 10^{-3}$ & $4.6 \cdot 10^{-3}$ to $\infty *$ & 0.97 & 87 & $\mathrm{nd}^{*}$ to 150 & 610 & n.d. $*$ to $\infty *$ & 620 & 1.02 \\
\hline Gabapentin & $1.8 \cdot 10^{-2}$ & $1.2 \cdot 10^{-2}$ to $2.7 \cdot 10^{-2}$ & 0.88 & 38 & 25 to 58 & 3500 & 2600 to $\infty *$ & 920000 & 260 \\
\hline Hydrochlorothiazide & $2.7 \cdot 10^{-1}$ & $1.2 \cdot 10^{-1}$ to $\infty *$ & 0.81 & 2.5 & nd* to 6 & 9600 & 4600 to $\infty *$ & 5100 & 0.53 \\
\hline Hydroxycotinine & $1.8 \cdot 10^{-2}$ & $1.2 \cdot 10^{-2}$ to $2.7 \cdot 10^{-2}$ & 0.88 & 38 & 26 to 60 & 350 & 260 to 480 & 460 & 1.31 \\
\hline Iopromide & $3.3 \cdot 10^{-2}$ & $2.0 \cdot 10^{-2}$ to $5.6 \cdot 10^{-2}$ & 0.79 & 21 & 12 to 34 & 52000 & 35000 to 80000 & 51000 & 0.99 \\
\hline Temazepam & $1.9 \cdot 10^{-2}$ & $1.6 \cdot 10^{-2}$ to $2.1 \cdot 10^{-2}$ & 0.98 & 37 & 33 to 43 & 1300 & 1200 to 1500 & 83 & 0.64 \\
\hline Tramadol & $2.9 \cdot 10^{-2}$ & $2.1 \cdot 10^{-2}$ to $4.0 \cdot 10^{-2}$ & 0.91 & 24 & 17 to 34 & 4000 & 3100 to 5100 & - & - \\
\hline Venlafaxine & $2.5 \cdot 10^{-2}$ & $1.9 \cdot 10^{-2}$ to $3.3 \cdot 10^{-2}$ & 0.93 & 27 & 21 to 36 & 2800 & 2300 to 3500 & 9200 & 3.3 \\
\hline
\end{tabular}

n.d.*: Denotes that the software was unable to calculate the lower boundary.

$\infty$ *: Denotes that the software was unable to calculate the upper boundary. 
Table S-15. Rate constant for chemical loss from the estuary segment derived by fitting the measured concentrations in river water to Eq. 4 (C: time invariant rate constant), Eq. 6 (UV: UV-radiation dependant constant). Eq. 8 ( $\mathrm{T}^{\mathrm{a}}$ : Temperature-dependant constant) for Type $\mathrm{C}$ compounds. Presented half-lives for the UV- and temperature models are based on the mean of the daily UV indices or water temperatures when water samples were taken.

\begin{tabular}{|c|c|c|c|c|c|c|}
\hline & \multicolumn{3}{|c|}{$k_{r}(1 /$ day $)$} & \multicolumn{2}{|c|}{ Half-life (days) } & \multirow[b]{2}{*}{ Approach } \\
\hline & Median & $95 \% \mathrm{CI}$ & $\mathrm{R}^{2}$ & Median & $95 \% \mathrm{CI}$ & \\
\hline \multirow{3}{*}{ 2,4-D } & $2.6 \cdot 10^{-2}$ & $2.3 \cdot 10^{-2}$ to $2.9 \cdot 10^{-2}$ & 0.99 & 27 & 24 to 30 & $\mathrm{C}$ \\
\hline & $2.3 \cdot 10^{-2}$ & $2.1 \cdot 10^{-2}$ to $2.5 \cdot 10^{-2}$ & 0.99 & 30 & 28 to 33 & UV \\
\hline & $3.2 \cdot 10^{-2}$ & $2.4 \cdot 10^{-2}$ to $4.2 \cdot 10^{-2}$ & 0.92 & 22 & 17 to 29 & $\mathrm{~T}^{\mathrm{a}}$ \\
\hline \multirow{3}{*}{ Diazinon } & $2.7 \cdot 10^{-3}$ & $1.8 \cdot 10^{-3}$ to $3.6 \cdot 10^{-3}$ & 0.61 & 260 & 190 to 400 & $\mathrm{C}$ \\
\hline & $2.5 \cdot 10^{-3}$ & $1.7 \cdot 10^{-3}$ to $3.4 \cdot 10^{-3}$ & 0.65 & 280 & 200 to 410 & UV \\
\hline & $7.8 \cdot 10^{-3}$ & $2.2 \cdot 10^{-3}$ to $1.6 \cdot 10^{-2}$ & 0.65 & 89 & 44 to 320 & $\mathrm{~T}^{\mathrm{a}}$ \\
\hline \multirow{3}{*}{ Dicamba } & $1.5 \cdot 10^{-2}$ & $9.0 \cdot 10^{-3}$ to $2.2 \cdot 10^{-2}$ & 0.72 & 45 & 31 to 77 & $\mathrm{C}$ \\
\hline & $1.5 \cdot 10^{-2}$ & $9.4 \cdot 10^{-3}$ to $2.1 \cdot 10^{-2}$ & 0.75 & 46 & 32 to 74 & UV \\
\hline & $6.2 \cdot 10^{-2}$ & $1.8 \cdot 10^{-2}$ to $1.8 \cdot 10^{-1}$ & 0.82 & 11 & 4 to 39 & $\mathrm{~T}^{\mathrm{a}}$ \\
\hline \multirow{3}{*}{ Methomyl } & $1.8 \cdot 10^{-2}$ & $1.4 \cdot 10^{-2}$ to $2.2 \cdot 10^{-2}$ & 0.92 & 39 & 31 to 49 & $\mathrm{C}$ \\
\hline & $1.9 \cdot 10^{-2}$ & $1.5 \cdot 10^{-2}$ to $2.3 \cdot 10^{-2}$ & 0.93 & 37 & 31 to 45 & UV \\
\hline & $3.8 \cdot 10^{-2}$ & $2.1 \cdot 10^{-2}$ to $6.3 \cdot 10^{-2}$ & 0.94 & 18 & 11 to 32 & $\mathrm{~T}^{\mathrm{a}}$ \\
\hline \multirow{3}{*}{ Prometryn } & $3.8 \cdot 10^{-3}$ & $2.9 \cdot 10^{-3}$ to $4.7 \cdot 10^{-3}$ & 0.79 & 180 & 150 to 240 & $\mathrm{C}$ \\
\hline & $3.8 \cdot 10^{-3}$ & $2.9 \cdot 10^{-3}$ to $4.9 \cdot 10^{-3}$ & 0.77 & 180 & 140 to 240 & UV \\
\hline & $2.2 \cdot 10^{-2}$ & $1.2 \cdot 10^{-2}$ to $3.8 \cdot 10^{-2}$ & 0.89 & 32 & 18 to 59 & $\mathrm{~T}^{\mathrm{a}}$ \\
\hline \multirow{3}{*}{ Tebuthiuron } & $5.1 \cdot 10^{-3}$ & $4.2 \cdot 10^{-3}$ to $6.0 \cdot 10^{-3}$ & 0.89 & 140 & 110 to 160 & $\mathrm{C}$ \\
\hline & $4.8 \cdot 10^{-3}$ & $3.7 \cdot 10^{-3}$ to $6.1 \cdot 10^{-3}$ & 0.89 & 140 & 110 to 190 & UV \\
\hline & $5.0 \cdot 10^{-3}$ & $4.2 \cdot 10^{-3}$ to $9.1 \cdot 10^{-3}$ & 0.89 & 140 & 76 to 160 & $\mathrm{~T}^{\mathrm{a}}$ \\
\hline
\end{tabular}




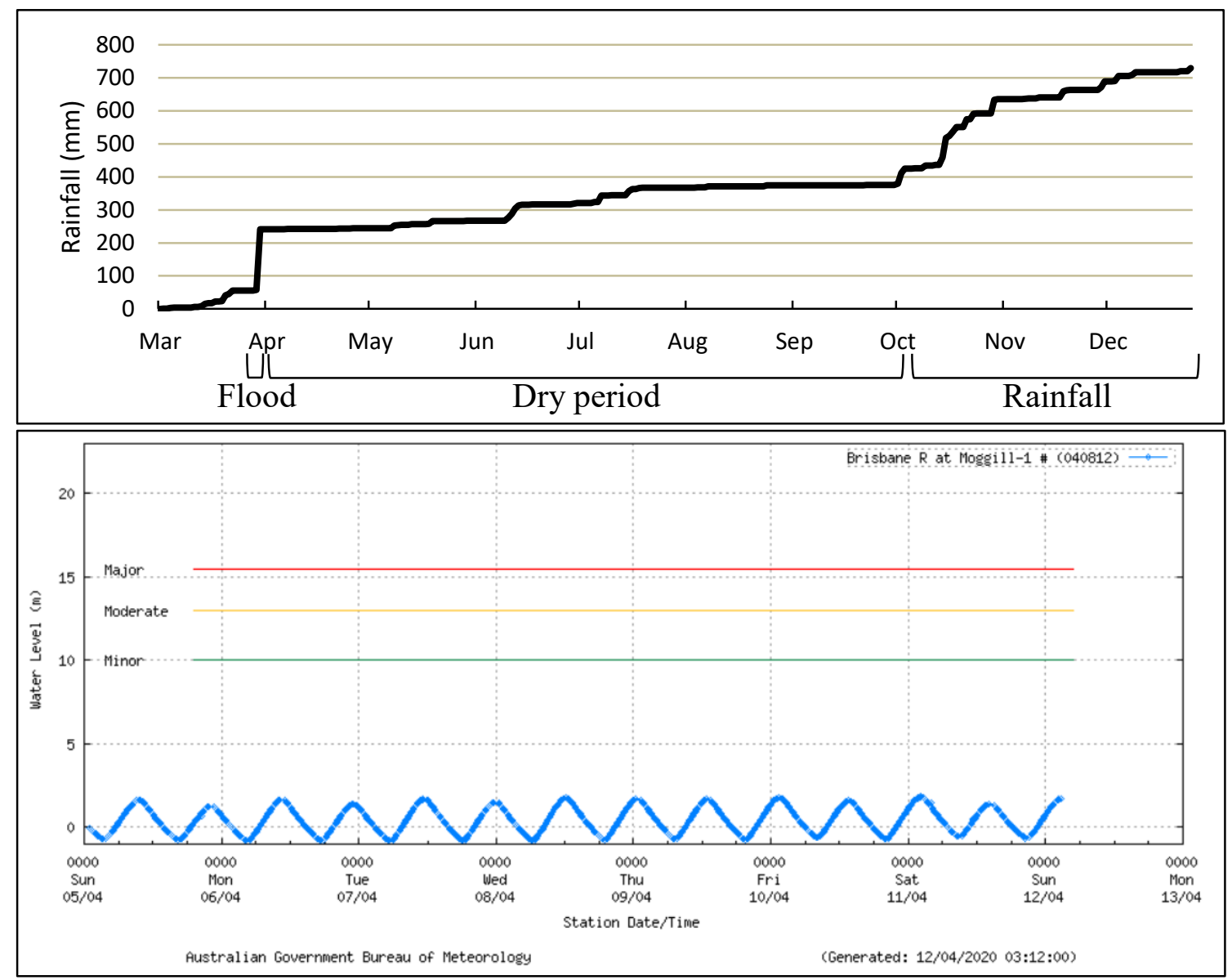

Figure S-1. Upper Panel: Cumulative rainfall at Savage's Crossing gauging station between March and December 2017 [1]. Lower Panel: Example of todal amplitude of the Brisbane River at Moggill between April 5 and April 122020. 

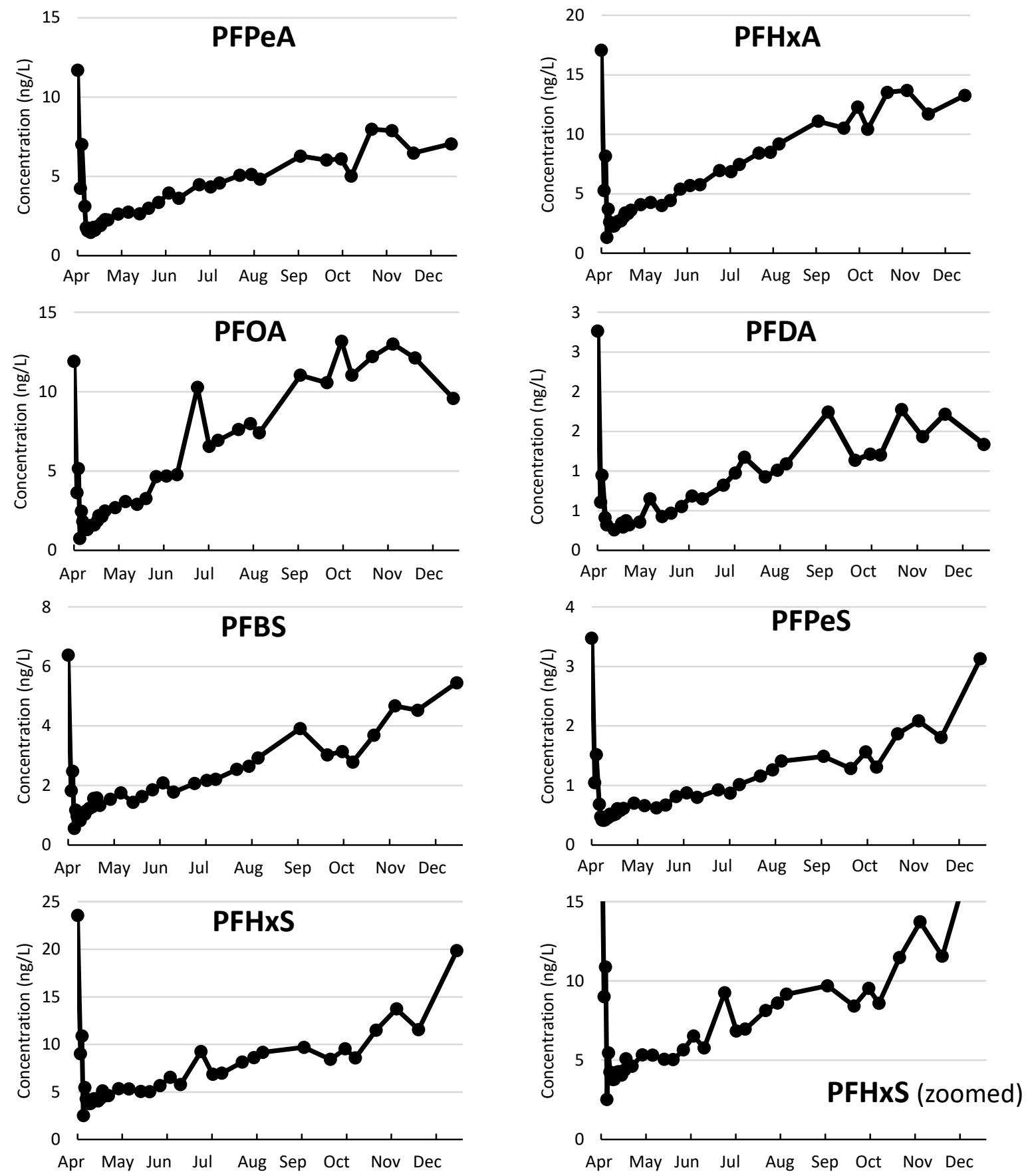

Figure S-2. Concentration of Type A chemicals during the whole sampling period. A zoom to the y-axis is provided for some substances to facilitate inspection of the trend. The $\mathrm{x}$-axis labels mark the beginning of the month. 

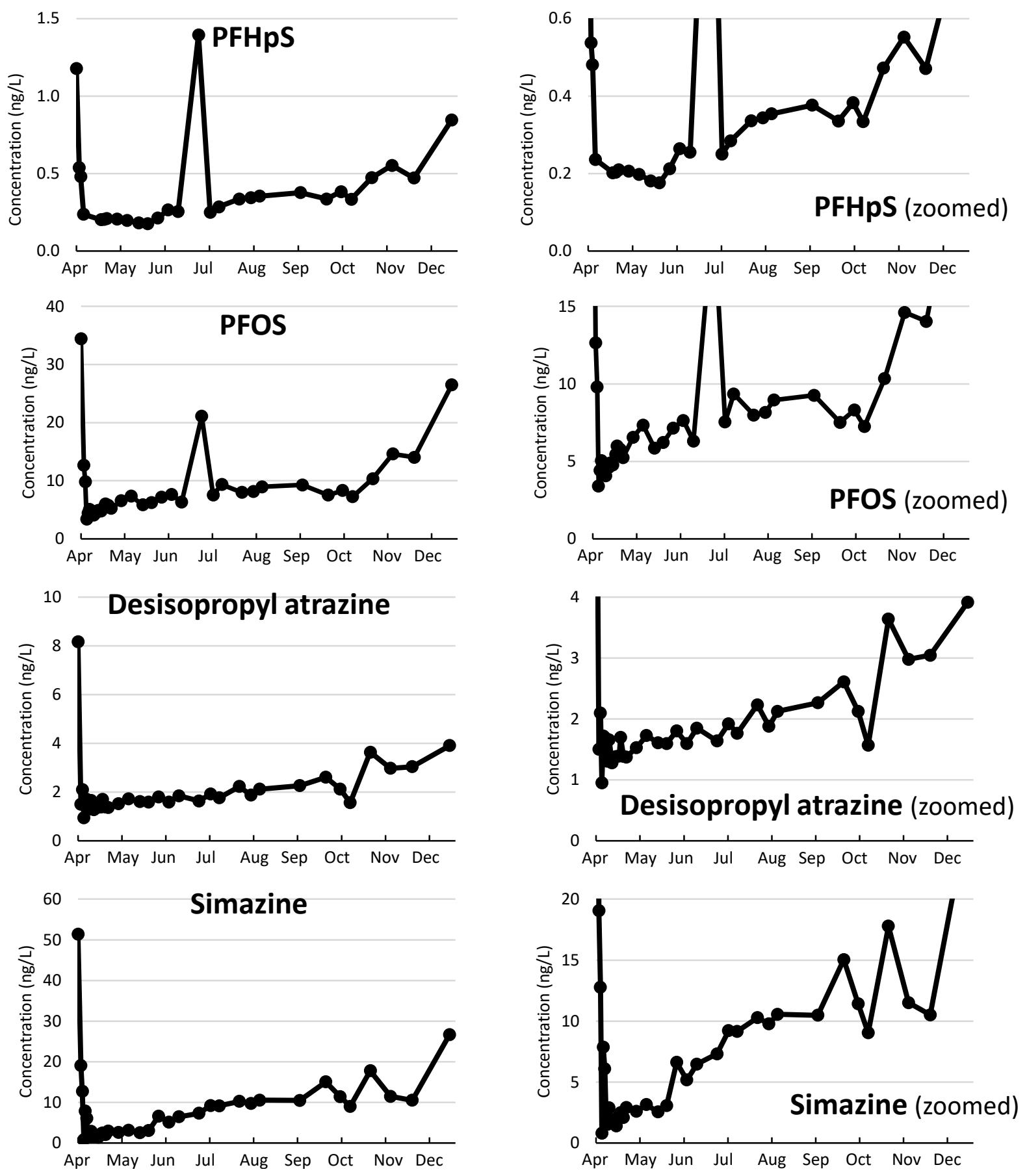

Figure S-2. (Continuation) Concentration of Type A chemicals during the whole sampling period. A zoom to the $\mathrm{y}$-axis is provided for some substances to facilitate inspection of the trend. The $\mathrm{x}$-axis labels mark the beginning of the month. 

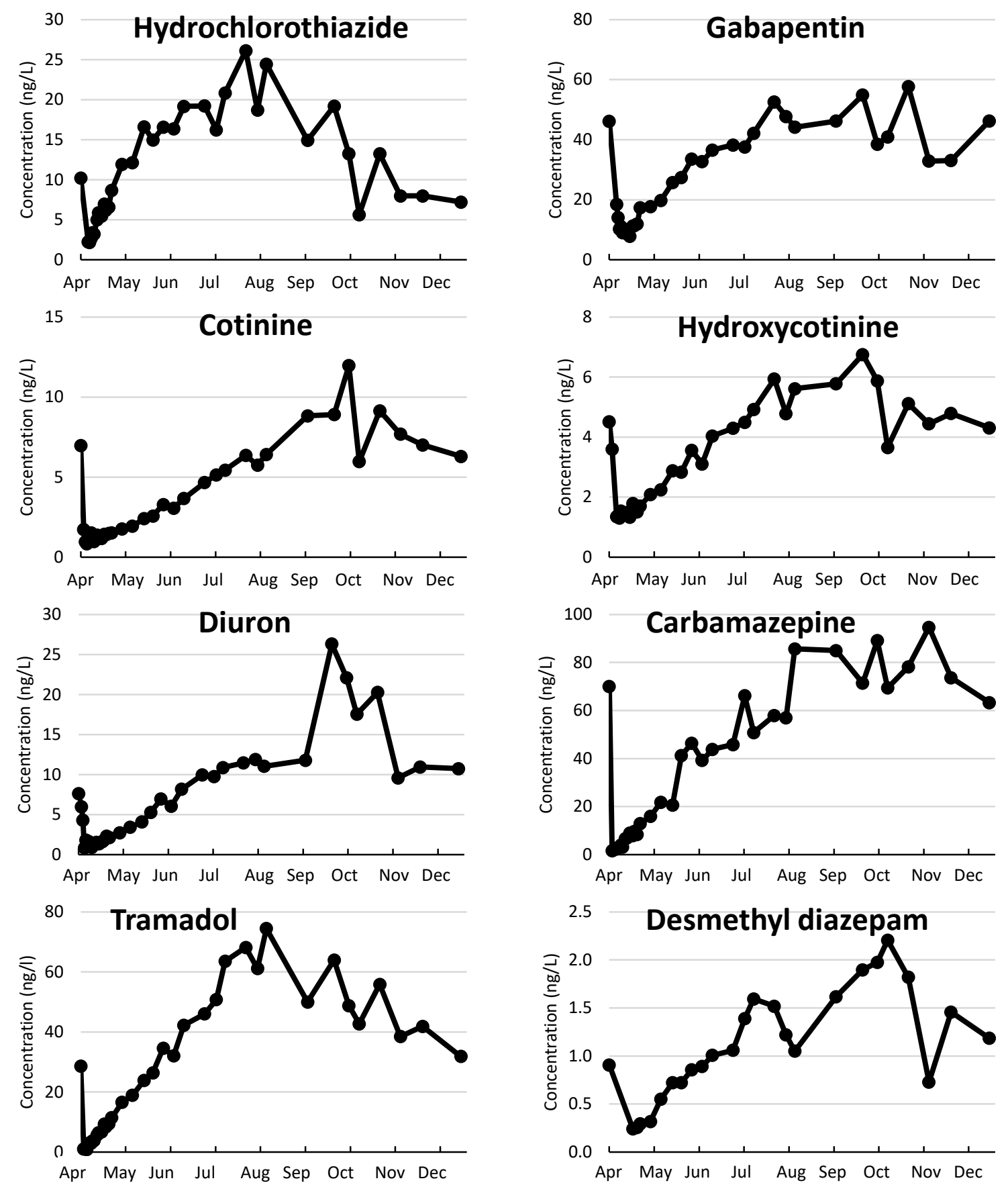

Figure S-3. Concentration of Type B chemicals during the whole sampling period. The $\mathrm{x}$-axis labels mark the beginning of the month. 


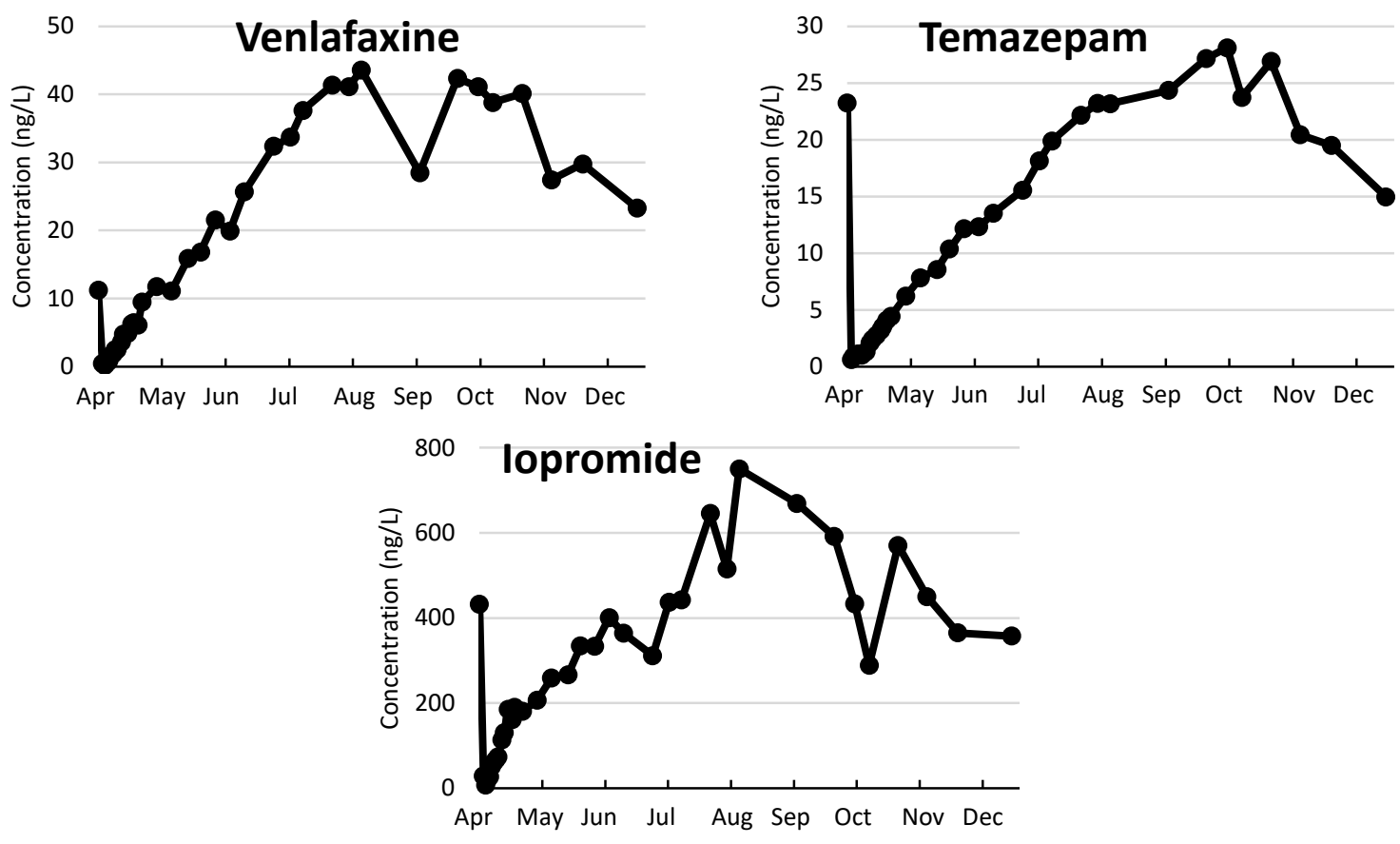

Figure S-3. (Continuation) Concentration of Type B chemicals during the whole sampling period. The xaxis labels mark the beginning of the month. 

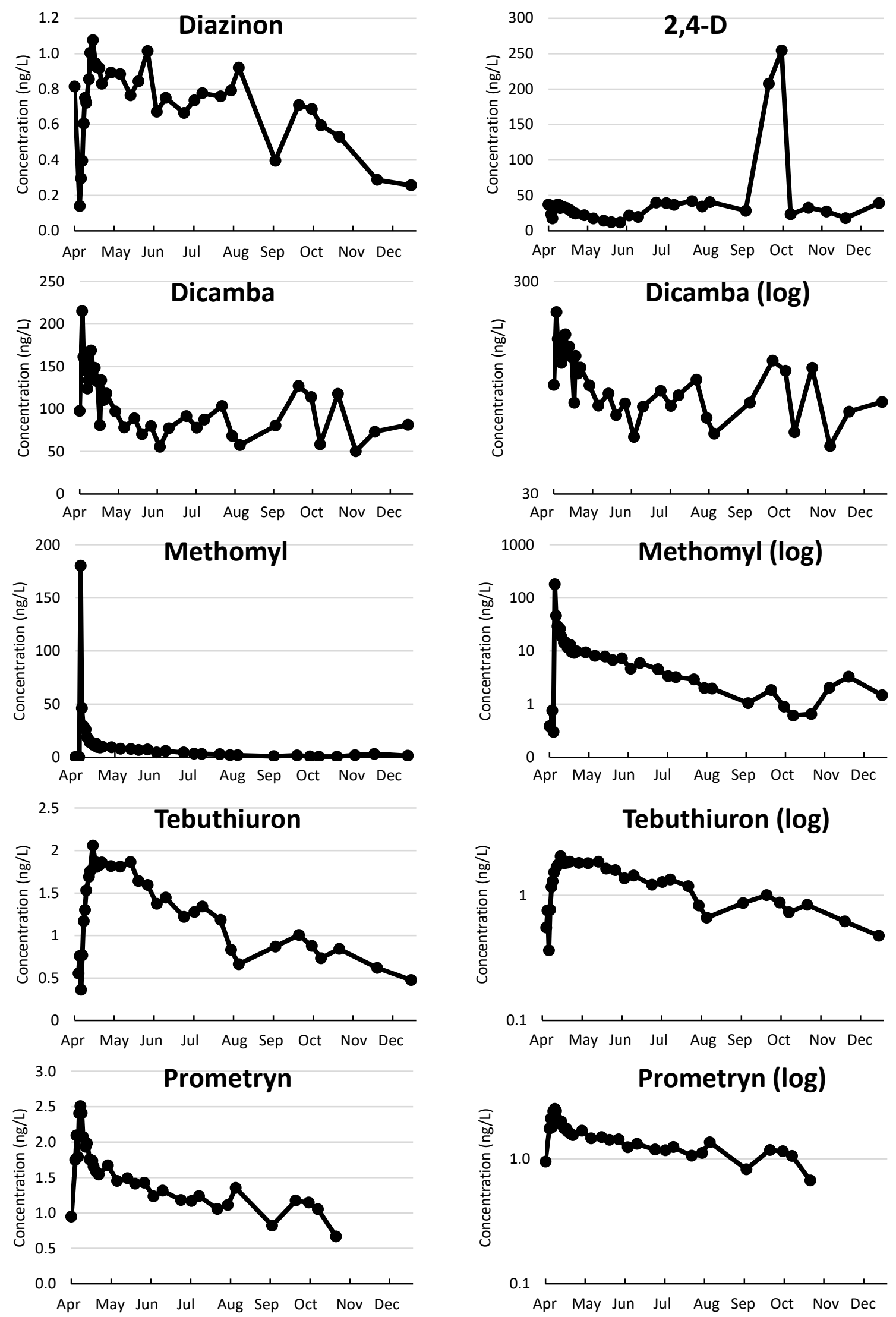

Figure S-4. Concentration of Type C chemicals during the whole sampling period. A semilogarithmic plot is provided for some substances to facilitate inspection of the elimination kinetics. The $\mathrm{x}$-axis labels mark the beginning of the month. 

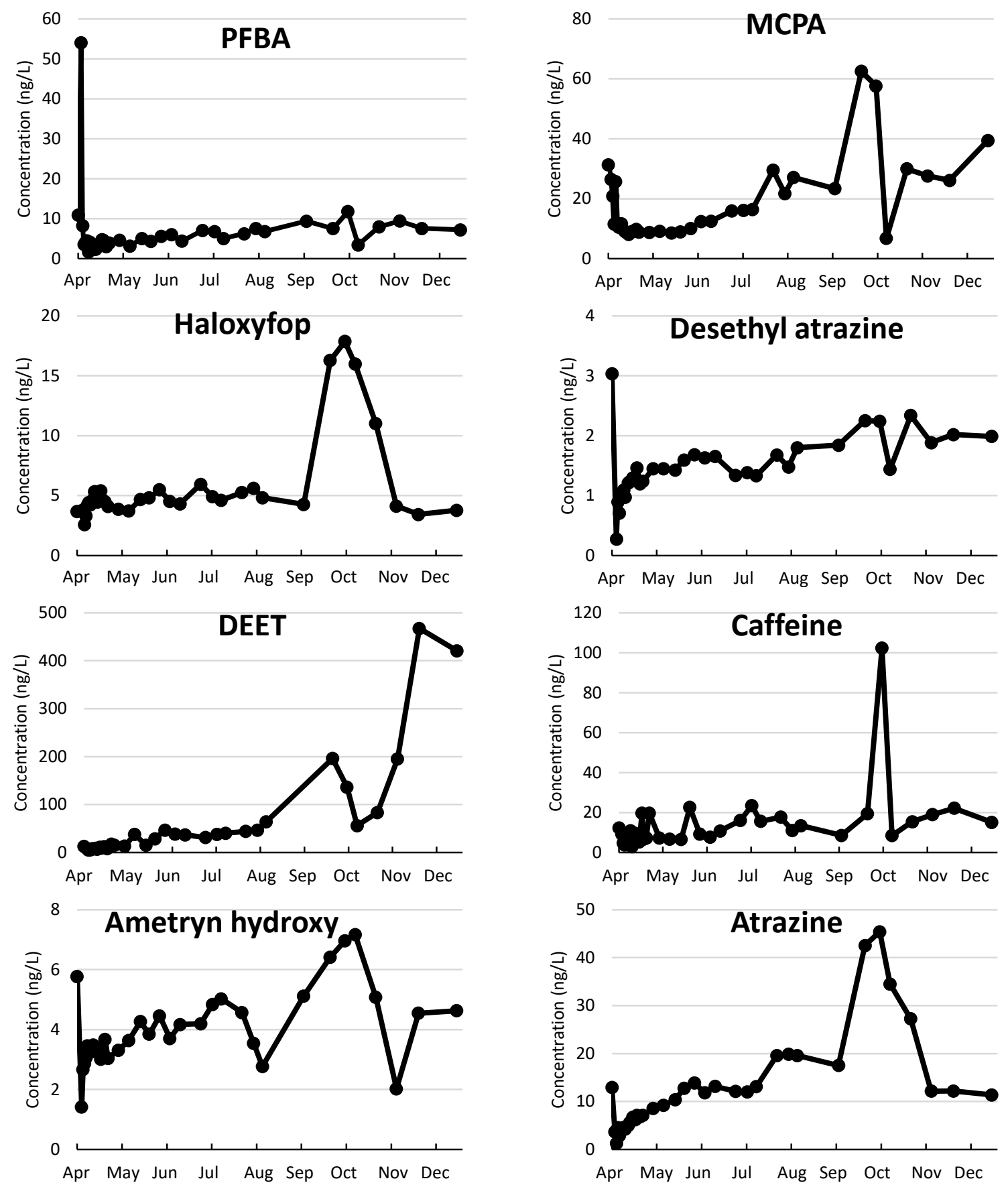

Figure S-5. Concentration trend during the whole sampling period for chemicals showing no consistent time treand. The $\mathrm{x}$-axis labels mark the beginning of the month. 

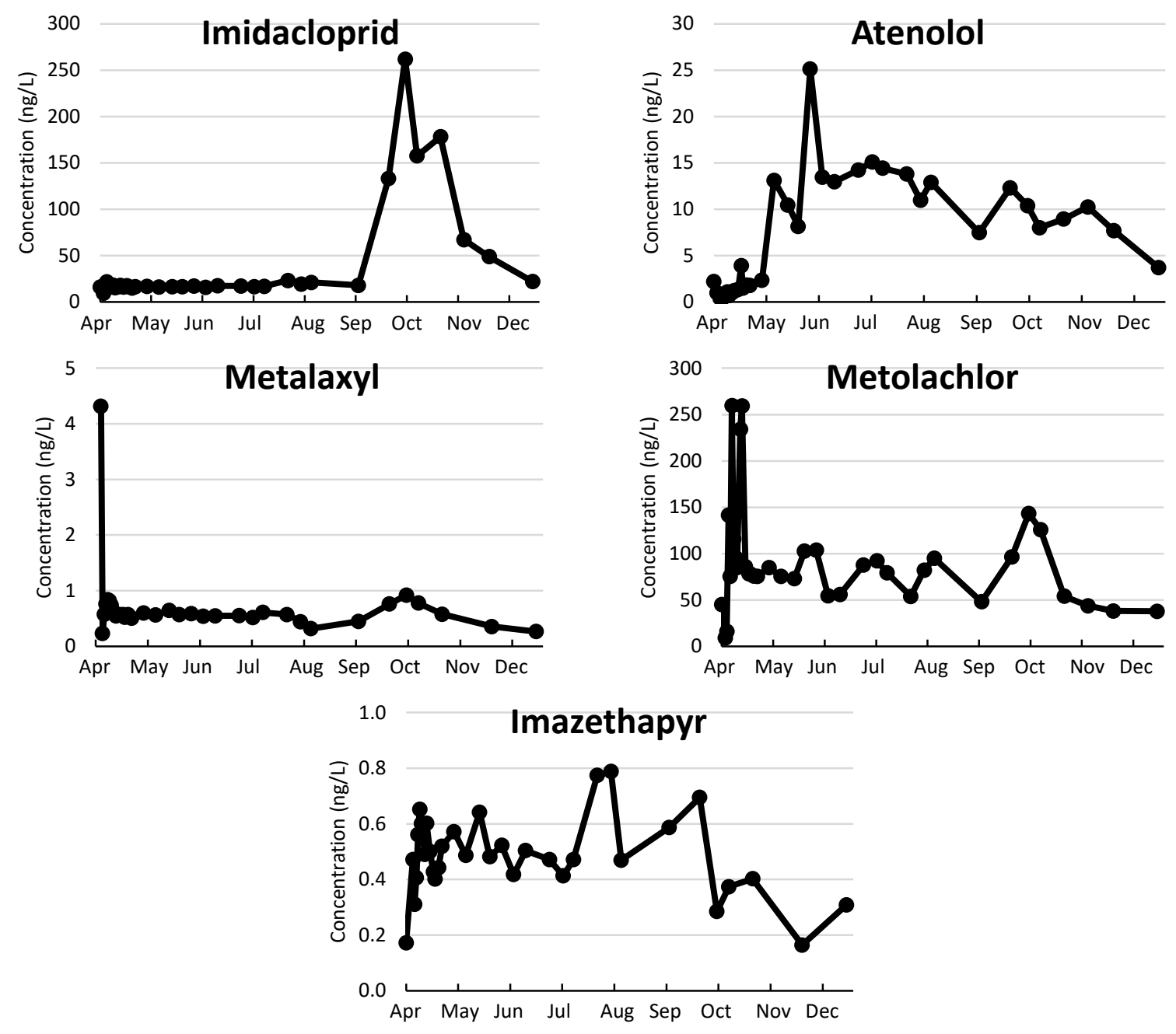

Figure S-5. (Continuation) Concentration trend during the whole sampling period for chemicals showing no consistent time trend. The $\mathrm{x}$-axis labels mark the beginning of the month. 

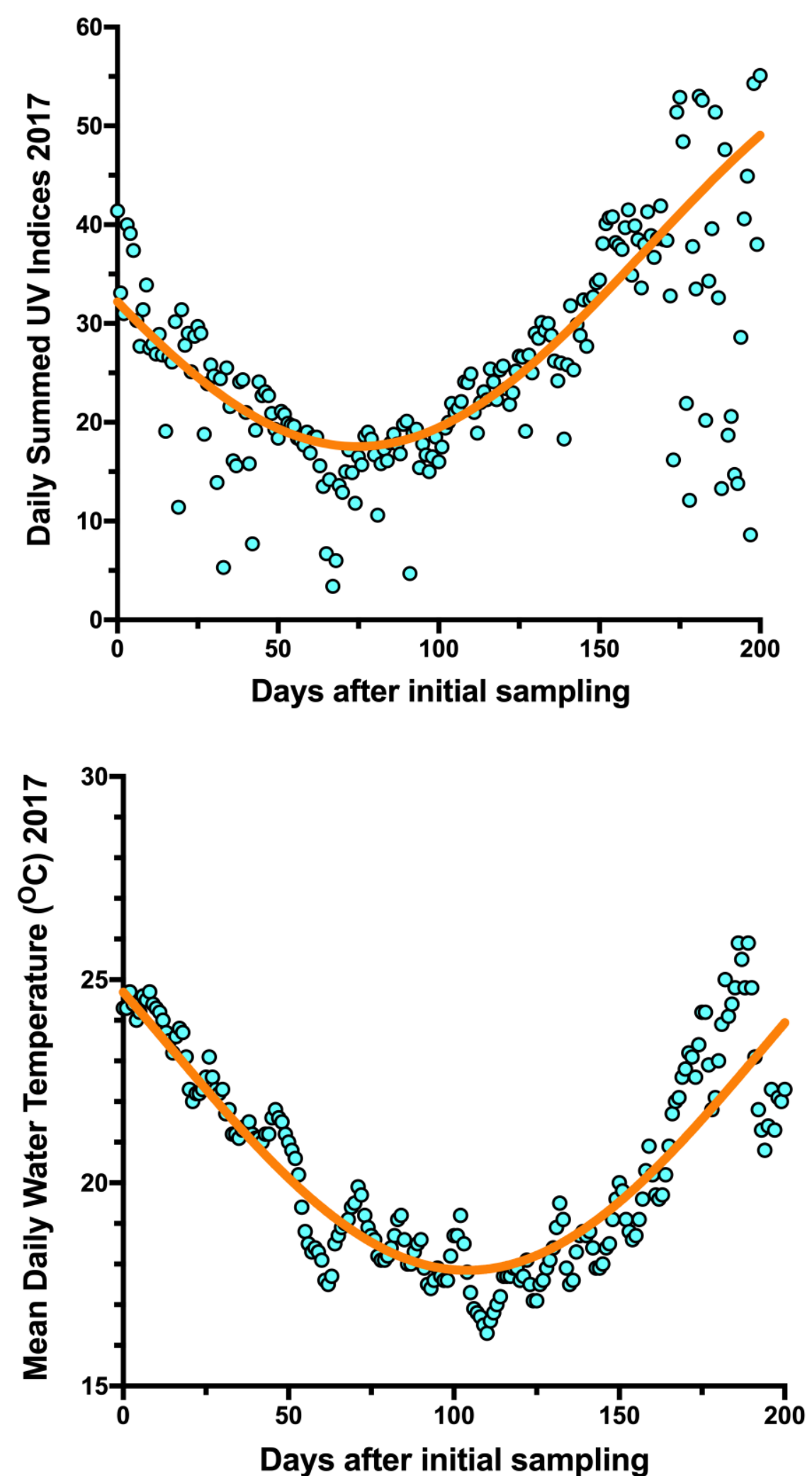

Figure S-6. Upper panel: Daily UV index at the Brisbane UV monitoring station, summarized from hourly UV index data [2] Lower panel: Daily average water temperature at the Savage's Crossing station on the Brisbane River [1]. The time axis of both graphs extends from April 7 (day 0) to October 22 (day 200) 2017. 


\section{References}

1. Water Monitoring Information at Savage's Crossing. Water Monitoring Information Portal. Queensland Goverment. https://water-

monitoring.information.qld.gov.au/host.htm?ppbm=143001C\&rs\&1\&rslf_org. [cited 2020 August].

2. Reference for the UV data. Australian Radiation Protection and Nuclear Safety Agency. Data request in August 2020 via https:/www.arpansa.gov.au/our-services/monitoring/ultravioletradiation-monitoring/special-requests-ultraviolet-data.

3. Bräunig, J., C. Baduel, A. Heffernan, A. Rotander, E. Donaldson and J.F. Mueller, Fate and redistribution of perfluoroalkyl acids through AFFF-impacted groundwater. Science of the Total Environment, 2017. 596-597: p. 360-368.

4. Gallen, C., C. Baduel, F.Y. Lai, K. Thompson, J. Thompson, M. Warne and J.F. Mueller, Spatiotemporal assessment of perfluorinated compounds in the Brisbane River system, Australia: Impact of a major flood event. Marine Pollution Bulletin, 2014. 85(2): p. 597-605.

5. Water Monitoring Information Portal: Queensland Goverment https://watermonitoring.information.qld.gov.au 2017 [cited 2020 Fenbruary].

6. Bureau of Meteorology: Australian goverment http://www.bom.gov.au/climate/current/annual/qld/archive/2017.brisbane.shtml\#recordsRainDaily High 2017 [cited 2020 February].

7. The Queensland Government and the Department of Defence. Per-and poly-fluoroalkyl substances (PFAS) contamination of waterways in the Ipswich area. 2018-2019 [cited 2020 April ].

8. Anim, A.K., K. Thompson, G.O. Duodu, B. Tscharke, G. Birch, A. Goonetilleke, G.A. Ayoko and J.F. Mueller, Pharmaceuticals, personal care products, food additive and pesticides in surface waters from three Australian east coast estuaries (Sydney, Yarra and Brisbane). Marine Pollution Bulletin, 2020. 153: p. 111014.

9. Anim, A.K., OCCURRENCE AND FATE OF SELECTED ORGANIC CONTAMINANTS IN SOILS,SEDIMENT AND ESTUARINE WATER FROM SOUTH-EAST QUEENSLAND. 2019, Queensland University of Technology.

10. Department of Defence-Australian Government. RAAF Base Amberley PFAS InvestigationDetailed Site Investigation. https://www.defence.gov.au/environment/pfas/amberley/. November 2018 [cited July 2020].

11. Baddiley BL, M.T., Braun C, Vardy S, Queensland Ambient PFAS Monitoring Program 20192020. Brisbane: Department of Environment and Science, Queensland Government. 2020.

12. Mussabek, D., L. Ahrens, K.M. Persson and R. Berndtsson, Temporal trends and sediment-water partitioning of per-and polyfluoroalkyl substances (PFAS) in lake sediment. Chemosphere, 2019. 227: p. 624-629.

13. Jeon, J., K. Kannan, B.J. Lim, K.G. An and S.D. Kim, Effects of salinity and organic matter on the partitioning of perfluoroalkyl acid (PFAs) to clay particles. Journal of Environmental Monitoring, 2011. 13(6): p. 1803-1810.

14. Messing, P., A. Farenhorst, D. Waite and J. Sproull, Influence of usage and chemical-physical properties on the atmospheric transport and deposition of pesticides to agricultural regions of Manitoba, Canada. Chemosphere, 2013. 90(6): p. 1997-2003.

15. Terrado, M., M. Kuster, D. Raldúa, M. Lopez De Alda, D. Barceló and R. Tauler, Use of chemometric and geostatistical methods to evaluate pesticide pollution in the irrigation and drainage channels of the Ebro river delta during the rice-growing season. Analytical and Bioanalytical Chemistry, 2007. 387(4): p. 1479-1488.

16. Prichard, T., J. Troiano, J. Marade, F. Guo and M. Canevari, Movement of Diuron and Hexazinone in Clay Soil and Infiltrated Pond Water. Journal of Environmental Quality, 2005. 34(6): p. 20052017. 
17. Seller, C., M. Honti, H. Singer and K. Fenner, Biotransformation of Chemicals in Water-Sediment Suspensions: Influencing Factors and Implications for Persistence Assessment. Environmental Science \& Technology Letters, 2020. 7(11): p. 854-860.

18. Minelgaite, G., A.H. Nielsen, M.L. Pedersen and J. Vollertsen, Photodegradation of three stormwater biocides. Urban Water Journal, 2017. 14(1): p. 53-60.

19. Yu, Y., H. Zhang and C. Lemckert, Salinity and turbidity distributions in the Brisbane River estuary, Australia. Journal of Hydrology, 2014. 519: p. 3338-3352.

20. Zou, H., M. Radke, A. Kierkegaard and M.S. McLachlan, Temporal Variation of Chemical Persistence in a Swedish Lake Assessed by Benchmarking. Environmental Science \& Technology, 2015. 49(16): p. 9881-9888.

21. West, C.E. and S.J. Rowland, Aqueous phototransformation of diazepam and related human metabolites under simulated sunlight. Environmental Science and Technology, 2012. 46(9): p. 4749-4756.

22. Bo, L., H. Liu and H. Han, Photocatalytic degradation of trace carbamazepine in river water under solar irradiation. Journal of Environmental Management, 2019. 241: p. 131-137.

23. Tixier, C., H.P. Singer, S. Oellers and S.R. Müller, Occurrence and Fate of Carbamazepine, Clofibric Acid, Diclofenac, Ibuprofen, Ketoprofen, and Naproxen in Surface Waters. Environmental Science \& Technology, 2003. 37(6): p. 1061-1068.

24. Zou, H., M. Radke, A. Kierkegaard, M. Macleod and M.S. McLachlan, Using chemical benchmarking to determine the persistence of chemicals in a swedish lake. Environmental Science and Technology, 2015. 49(3): p. 1646-1653.

25. Yu, H.-W., M. Park, S. Wu, I.J. Lopez, W. Ji, J. Scheideler and S.A. Snyder, Strategies for selecting indicator compounds to assess attenuation of emerging contaminants during $U V$ advanced oxidation processes. Water Research, 2019. 166: p. 115030.

26. Kalsch, W., Biodegradation of the iodinated X-ray contrast media diatrizoate and iopromide. Science of the Total Environment, 1999. 225(1-2): p. 143-153.

27. Reeves, R.R. and S.K. Cox, Similar effects of tramadol and venlafaxine in major depressive disorder. Southern medical journal, 2008. 101(2): p. 193-195.

28. Gulde, R., S. Anliker, H.-P.E. Kohler and K. Fenner, Ion Trapping of Amines in Protozoa: A Novel Removal Mechanism for Micropollutants in Activated Sludge. Environmental Science \& Technology, 2018. 52(1): p. 52-60.

29. Mackie, R.S., B.J. Tscharke, J.W. O'Brien, P.M. Choi, C.E. Gartner, K.V. Thomas and J.F. Mueller, Trends in nicotine consumption between 2010 and 2017 in an Australian city using the wastewater-based epidemiology approach. Environment International, 2019. 125: p. 184-190.

30. Davis, R.A. and M. Curvali, Chapter 14 - Determination of nicotine and its metabolites in biological fluids: in vivo studies, in Analytical Determination of Nicotine and Related Compounds and their Metabolites, J.W. Gorrod and P. Jacob, Editors. 1999, Elsevier Science: Amsterdam. p. 583-643.

31. National Center for Biotechnology Information (2021). PubChem Annotation Record for Cotinine, Source: Hazardous Substances Data Bank (HSDB). Retrieved January 8, 2021 from https://pubchem.ncbi.nlm.nih.gov/source/hsdb/7805.

32. Bradley, P.M., L.B. Barber, D.W. Kolpin, P.B. McMahon and F.H. Chapelle, Biotransformation of caffeine, cotinine, and nicotine in stream sediments: Implications for use as wastewater indicators. Environmental Toxicology and Chemistry, 2007. 26(6): p. 1116-1121.

33. Van Scoy, A.R., M. Yue, X. Deng and R.S. Tjeerdema, Environmental Fate and Toxicology of Methomyl, in Reviews of Environmental Contamination and Toxicology, D.M. Whitacre, Editor. 2013, Springer New York: New York, NY. p. 93-109.

34. Tien, C.-J., M.-C. Lin, W.-H. Chiu and C.S. Chen, Biodegradation of carbamate pesticides by natural river biofilms in different seasons and their effects on biofilm community structure. Environmental Pollution, 2013. 179: p. 95-104. 
35. National Center for Biotechnology Information. PubChem Database. TEBUTHIURON, Source=Hazardous Substances Data Bank (HSDB),

https://pubchem.ncbi.nlm.nih.gov/source/hsdb/6863\#section=Human-Health-Effects (accessed on July 21, 2020).

36. Mercurio, P., J.F. Mueller, G. Eaglesham, J. O'Brien, F. Flores and A.P. Negri, Degradation of herbicides in the tropical marine environment: Influence of light and sediment. PLoS ONE, 2016. 11(11).

37. Liu, J., D. Pan, X. Wu, H. Chen, H. Cao, Q.X. Li and R. Hua, Enhanced degradation of prometryn and other s-triazine herbicides in pure cultures and wastewater by polyvinyl alcohol-sodium alginate immobilized Leucobacter sp. JW-1. Science of the Total Environment, 2018. 615: p. 7886.

38. Pérez-Bárcena, J.F., D. Ahuatzi-Chacón, K.L. Castillo-Martínez, N. Ruiz-Ordaz, J. GalíndezMayer, C. Juárez-Ramírez and O. Ramos-Monroy, Effect of herbicide adjuvants on the biodegradation rate of the methylthiotriazine herbicide prometryn. Biodegradation, 2014. 25(3): $\mathrm{p}$. 405-415.

39. Edwards, D., Reregistration Eligibility Decision (RED) Diazinon EPA 738-R-04-006. 2006, U.S. Environmental Protection Agency, Office of Prevention, Pesticides and Toxic Substances, Office of Pesticide Programs, U.S. Government Printing Office: Washington, DC.

40. HSBD. Diazinon https://pubchem.ncbi.nlm.nih.gov/source/hsdb/303. April 2020].

41. Degenhardt, D., A.J. Cessna, R. Raina, A. Farenhorst and D.J. Pennock, Dissipation of six acid herbicides in water and sediment of two Canadian prairie wetlands. Environmental Toxicology and Chemistry, 2011. 30(9): p. 1982-1989. 